\title{
The Blattodea s.s. (Insecta, Dictyoptera) of the Guiana Shield
}

\author{
Dominic A. Evangelista', Kimberly Chan', Kayla L. Kaplan', \\ Megan M. Wilson', Jessica L. Ware' \\ I Department of Biology, Rutgers University, 195University Ave, Newark, NJ, 07102, USA \\ Corresponding author: Dominic A. Evangelista (dominicev@gmail.com)
}

Academic editor: Sam Heads | Received 13 May 2014 | Accepted 11 November 2014 | Published 22 January 2015

http://zoobank.org/C4ACAF17-E887-406A-AF7C-6D0155E7F392

Citation: Evangelista DA, Chan K, Kaplan KL, Wilson MM, Ware JL (2015) The Blattodea s.s. (Insecta, Dictyoptera) of the Guiana Shield. ZooKeys 475: 37-87. doi: 10.3897/zookeys.475.7877

\begin{abstract}
Here we provide a checklist of cockroach species known from areas within the Guiana Shield based on literature records and new field collection. We give records of sixteen species collected in Guyana, eight of which are new records for Guyana and one of which is a new generic record for the entire Guiana Shield. We also provide a description for a geographically disparate species of Calhypnorna Stal, and the new species Xestoblatta berenbaumae. The complete checklist contains 234 species of Blattodea s.s. currently known in the shield. This checklist shows particularly low richness in Guianan Venezuela, Roraima and Amapa Brazil, but this is likely an artifact due to under-sampling. Indeed, based on previously published data and current fieldwork, we believe that most regions of the Guiana Shield are under-sampled for cockroaches. Despite this, French Guiana (151 spp.) and Suriname (136 spp.) rank as the second and sixth most species dense faunas of cockroaches in the neotropics.
\end{abstract}

\section{Keywords}

Cockroach, species richness, Calhypnorna, Xestoblatta, Guyana

\section{Introduction}

The Guiana Shield is known for a high diversity of both plant and animal life (Alexander et al. 2005). Blattodea (Insecta: Dictyoptera), or cockroaches and termites, as well as most other insects, remain under-sampled relative to their biodiversity in the region. Developing more complete lists of fauna improves our ability to infer biogeographical patterns and make predictions about biodiversity loss. Additionally, keeping current 
records of regional faunas can assist in documenting introduced and invasive species, something particularly relevant to the study of cockroaches (Evangelista et al. 2013; Nickle 1984; Peterson and Cobb 2009).

The cockroach fauna of the entire Guiana Shield has previously been addressed by three works (i.e. Bonfils 1975; Bruijning 1959; Princis 1963). Princis' catalogue (1963) of global cockroach distributions is an important resource to consult for this fauna. However, there were cases (although very few) where Princis was incomplete in his records (pers. obs.; Pellens and Grandcolas 2008). Bruijning's (1959) and Bonfils' (1975) checklists are more manageable than Princis's global catalog given their focused geographic scope, but they are also an incomplete record of the fauna. Regardless, Bonfils' (1975), Bruijning's (1959) and Princis' (1963) work are all now 40 years or more out of date.

The cockroach fauna of sections of the Guiana Shield have been addressed directly by a few sources (e.g., Bonfils 1987; Bruijning 1959; Hebard 1926; Perez 1988; Rehn 1906; Rocha E Silva Albuquerque and Gurney 1962) as well as peripherally by others (e.g., Evangelista et al. 2014; Hebard 1921b; 1929; Pellens and Grandcolas 2008; Rehn 1928; Velez 2008). A few manuscripts have addressed the Blattodean faunas of French Guiana (Hebard 1926) and Suriname (Bruijning 1959) respectively. The Guianan fauna of relevant parts of Brazil and Venezuela are available from checklists for these respective countries (Bonfils 1987; Pellens and Grandcolas 2008; Perez 1988). However, there is no singular source to be consulted for the Blattodean fauna of Guyana (formerly known as British Guyana).

Lastly, the most current phylogenies of Blattodea all show that termites (Termitoidae) are nested within Blattodea (Djernaes et al. 2012, 2014; Inward et al. 2007; Ware et al. 2008). Given that this has only been recently adopted by systematists, there are few taxonomic treatments considering both termites and cockroaches simultaneously. Since each insect group requires very different morphological and organismal expertise this is understandable. In following, we present the most recent summative list of the non-termite Blattodea fauna of the Guiana Shield as well as for the country of Guyana.

\section{Methods}

\section{Checklist}

The checklist was initially compiled by synthesizing range data from the published literature. Searches for taxonomic records included some combination of the following locality names: British Guiana, Suriname, French Guiana, Guyane, Guiana or Guyana. Five additional sources were consulted (Bonfils 1987; Lopez-Osorio and MirandaEsquivel 2010; Pellens and Grandcolas 2008; Perez 1988) for the taxa of the following states: Amazonas Venezuela, Bolivar Venezuela, Delta Amacuro Venezuela, Roraima Brazil and Amapa Brazil. The states of Para and Amazonas in Brazil were omitted because the majority of these states do not fall within the borders of the Guiana Shield. The recently published checklist of the cockroaches of Brazil (Pellens and Grandcolas 2008) sufficiently covered the fauna of these states. We treated ranges specified by 
Princis (1963) as circumtropical, neotropical, or cosmopolitan as a presence for each region, even without a specific record for that region. Additional records were added based on specimens collected by the Ware lab in the field.

The validity of all taxonomic names was verified on the Cockroach Species File (CSF) online database (Beccaloni 2014). All synonymous names were changed to their valid name in the final checklist. All invalid higher taxa were given proper names in accordance with the most current taxonomy (Beccaloni and Eggleton 2011, 2013).

\section{Specimen collection}

We collected cockroaches from the field on four occasions from 2011 to 2013. All specimens were collected in Guyana. Specific collection information (locality and GPS, collection date, collectors and ecological information) is given with each record.

\section{New records and descriptions}

Species that were collected and could be identified are presented here. We report all collection information and some morphological information for each specimen as well as currently known geographic distribution as described on the Cockroach Species File database (Beccaloni 2014). All morphological measurements were done using Infinity software (INFINITY Camera Software 2013). For new species, we provide descriptions of gross morphology and male genitalia. The genitalia were dissected in accordance with the method of Roth (1969), whereby the genitalia are removed from the specimen by making a lateral incision along the subgenital plate, separating the genitalia from the remainder of the body and placing them in a $\mathrm{KOH}(10 \%$ by mass) solution until cleared (approx. 8 hours). Cleared genitalia were kept in a micro-vial with $70 \%$ ethanol after examination. We also include some notes on potential evolutionary relationships of some genera by referencing the cytochrome oxidase I (COI) gene tree published by the first and last author (Evangelista et al. 2014).

We imported the checklist data into Mathematica 9.1 (Wolfram Research 2012) to calculate the endemism rates of the faunas of each region. We calculated this as the proportion of species in a given region not present in any other region of the shield. We also calculated faunal similarity rates (inverse of endemism) among each region.

\section{Results}

\section{Records and descriptions of cockroaches from Guyana}

Here we report information on some of the specimens from our field collection. Those species listed here that are new records for Guyana are denoted by a "+" in the checklist (Table 1). Morphological measurements for all specimens are given in Table 2. 


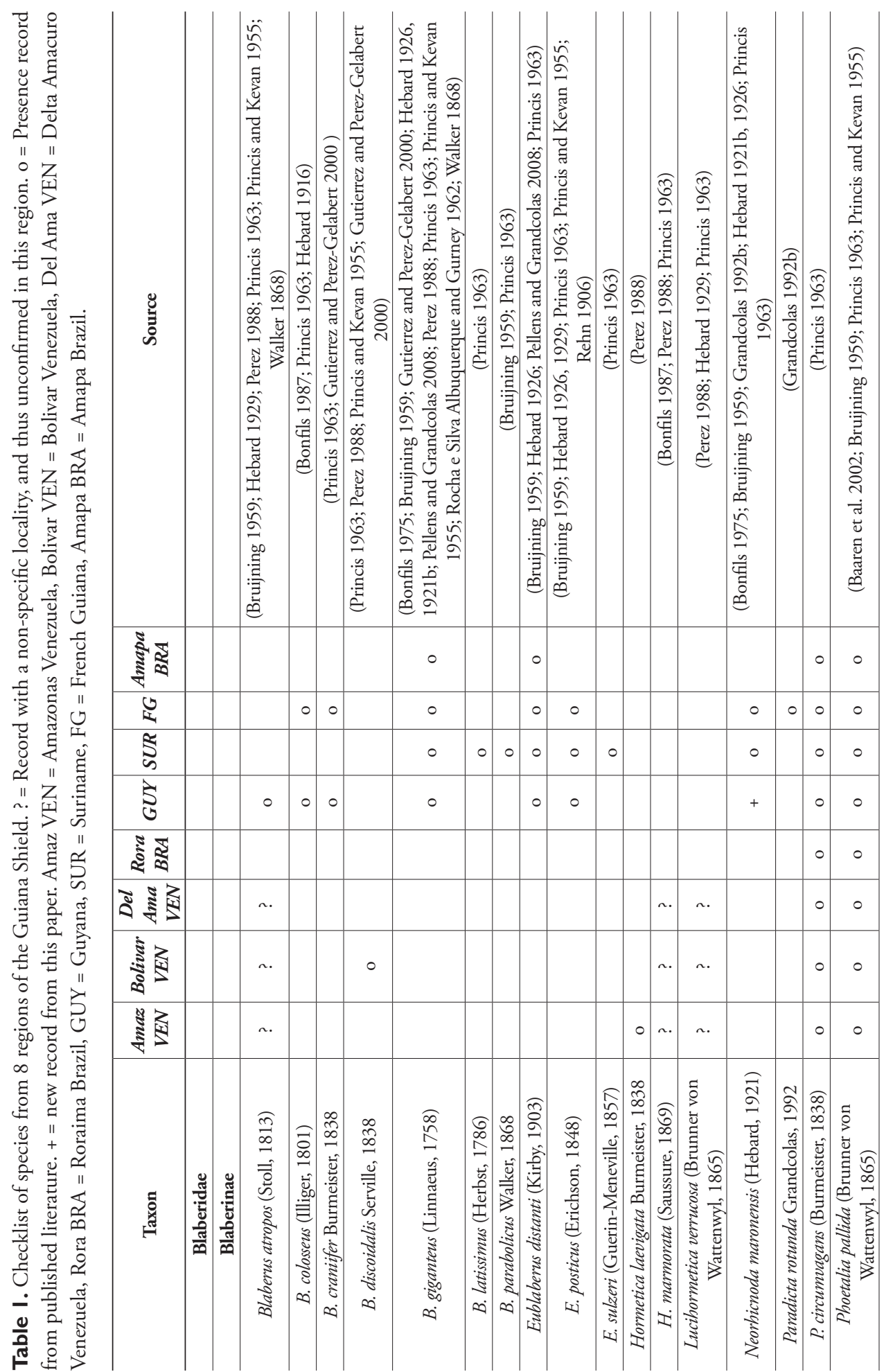




\begin{tabular}{|c|c|c|c|c|c|c|c|c|c|c|c|c|c|c|c|c|c|c|c|}
\hline 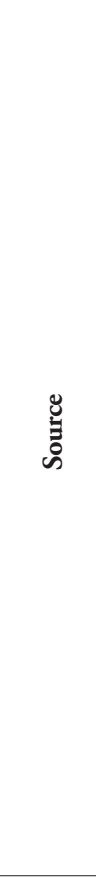 & 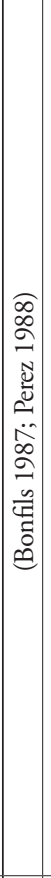 & 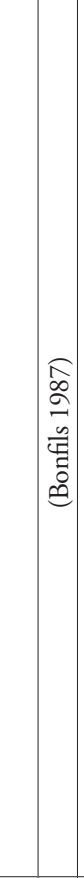 & 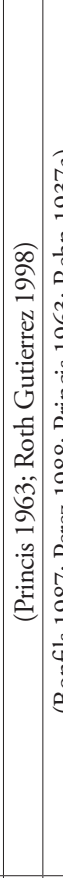 & 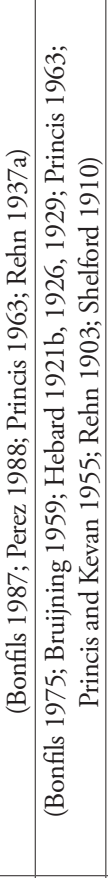 & 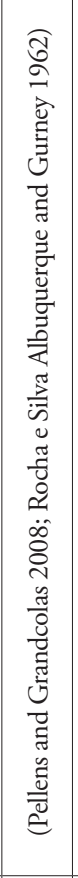 & 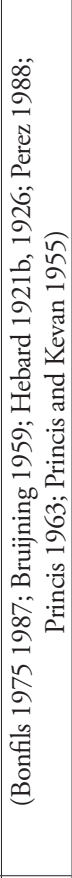 & 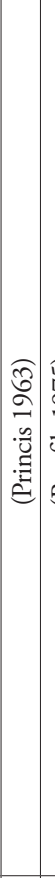 & 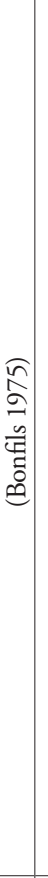 & 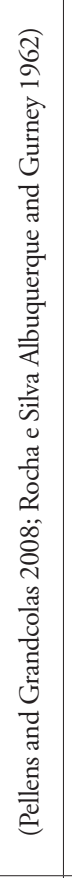 & 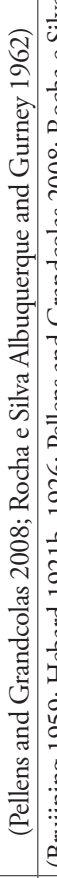 & 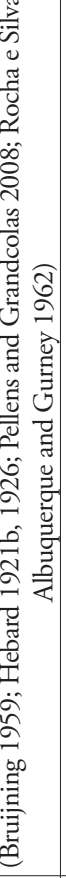 & 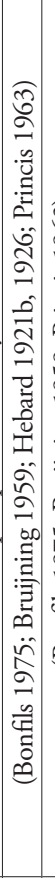 & 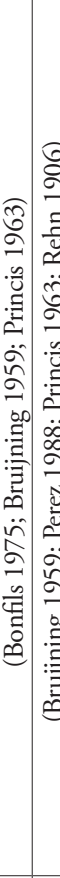 & 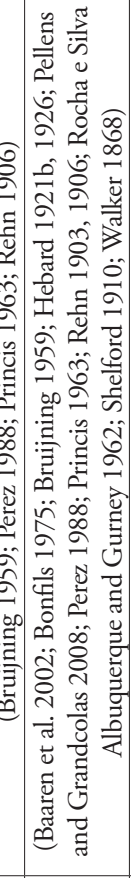 & 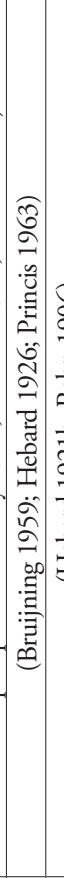 & $\begin{array}{l} \\
\\
\end{array}$ & 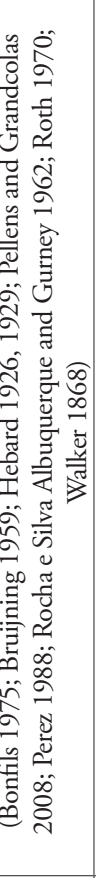 & 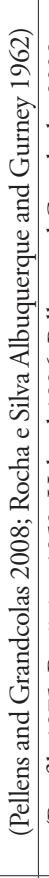 & 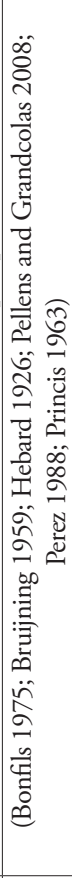 \\
\hline క్ & & & & & $\circ$ & & & & 0 & 0 & 0 & & & $\circ$ & & & 0 & 0 & \\
\hline U. & & & & $\circ$ & & $\circ$ & & 0 & & & 0 & 0 & 0 & $\circ$ & 0 & 0 & 0 & & 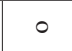 \\
\hline క & & & 0 & $\circ$ & & $\circ$ & & & & & $\circ$ & 0 & $\begin{array}{lll}0 & 0\end{array}$ & $\circ$ & 0 & & $\circ$ & & 0 \\
\hline S & & & 0 & 0 & & & 0 & & & & & & & $\circ$ & 0 & ○ & 0 & & + \\
\hline בิ & & & & & & & & & & & & & & & & & & & \\
\hline จั & . & & & & & & & & & & & & $n$ & n. & & & & & \\
\hline : & $\sim$ & 0 & & & & & & & & & & & n & n. & & & & & \\
\hline 논 & $n$. & & & 0 & & 0 & & & & & & & & n. & & & $n$. & & $\circ$ \\
\hline 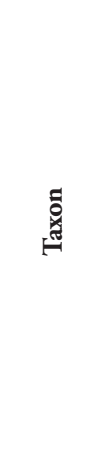 & 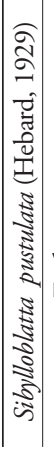 & 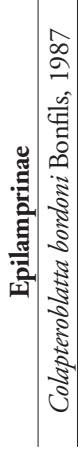 & 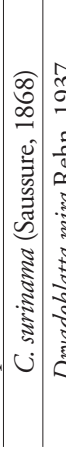 & 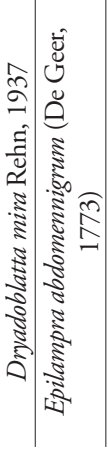 & 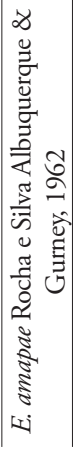 & 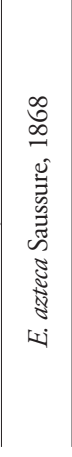 & 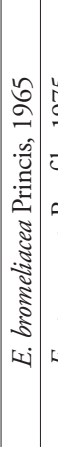 & 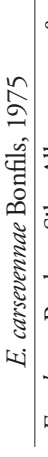 & 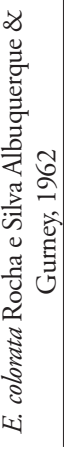 & 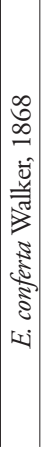 & 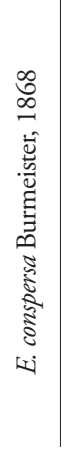 & 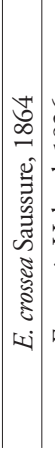 & 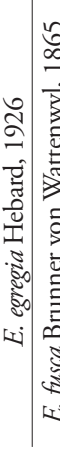 & 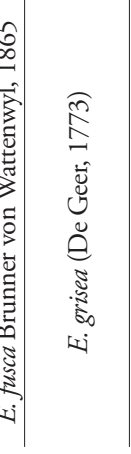 & 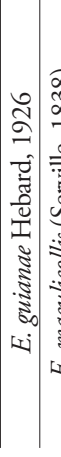 & 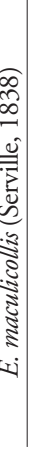 & 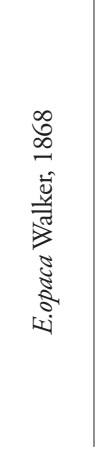 & 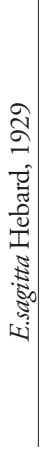 & 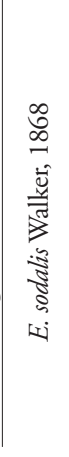 \\
\hline
\end{tabular}




\begin{tabular}{|c|c|c|c|c|c|c|c|c|c|c|c|c|c|c|c|c|c|c|c|c|}
\hline ڤั & 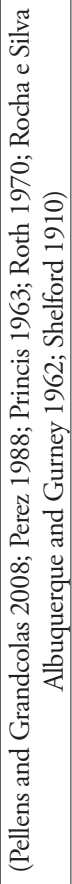 & 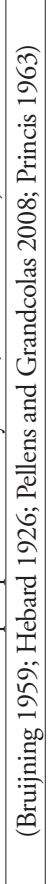 & 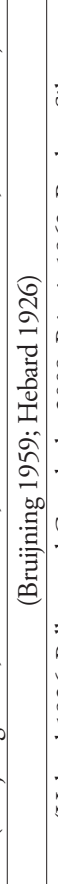 & 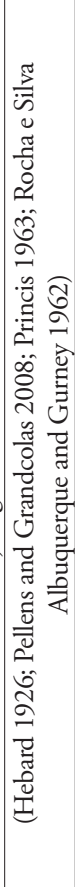 & 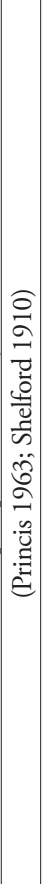 & 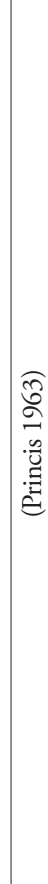 & 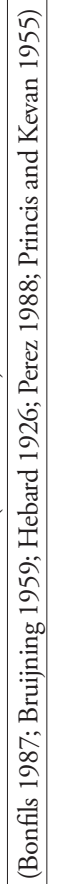 & 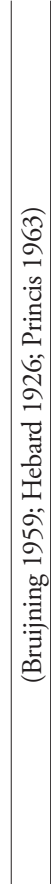 & 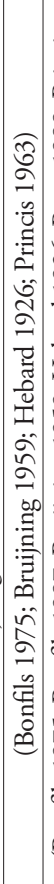 & 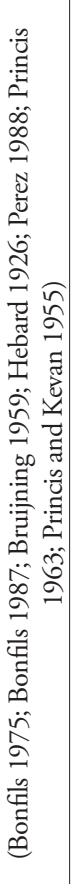 & 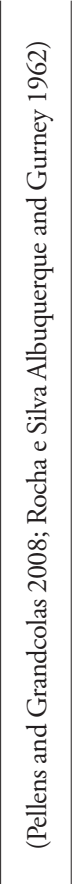 & 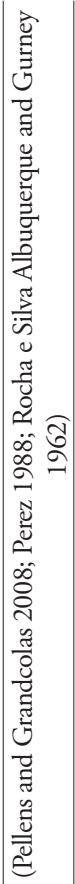 & 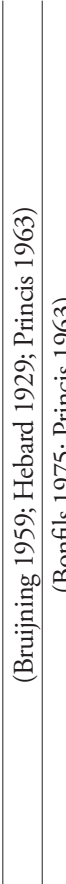 & 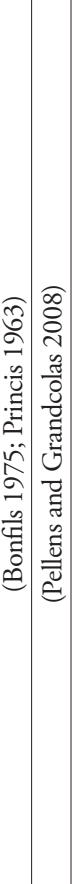 & 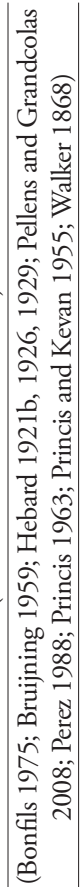 & 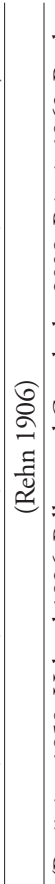 & 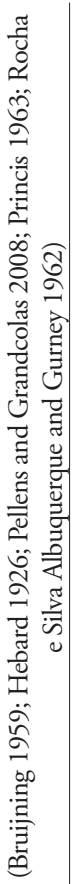 & 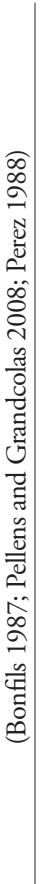 & 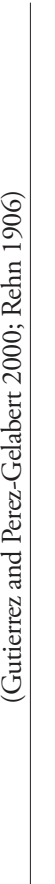 & 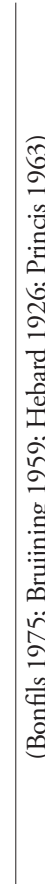 \\
\hline 촌 & $\circ$ & 0 & & $\circ$ & & $\circ$ & 0 & & & & $\circ$ & $\circ$ & & & $\circ$ & & $\circ$ & 0 & & \\
\hline v & & $\circ$ & 0 & $\circ$ & & $\circ$ & 0 & $\circ$ & 0 & $\circ$ & & & & 0 & 0 & & 0 & & & 0 \\
\hline క్ & & 0 & 0 & 0 & 0 & $\circ$ & 0 & 0 & 0 & $\circ$ & & & 0 & 0 & 0 & & 0 & & & 0 \\
\hline Ș & & & & & & $\circ$ & 0 & 0 & 0 & & & & 0 & 0 & 0 & 0 & & & 0 & \\
\hline 范 & & & & & & 0 & 0 & & & & & & & $\circ$ & 0 & & & & & \\
\hline จั & & & & & & $\circ$ & 0 & & & & & $n$. & & & 0 & & & & & \\
\hline : & & & & & & $\circ$ & 0 & & & & & n. & & & 0 & & & 0 & & \\
\hline 蛋 & 0 & & & & & $\circ$ & 0 & & & 0 & & n. & & & 0 & & & 0 & & \\
\hline 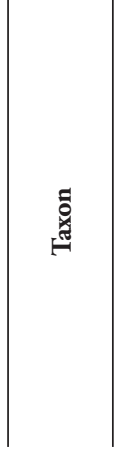 & 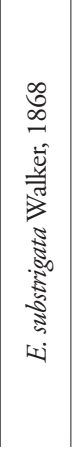 & 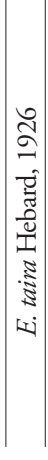 & 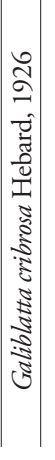 & 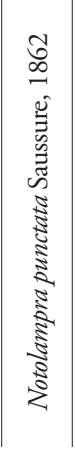 & 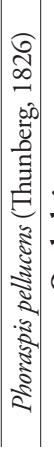 & 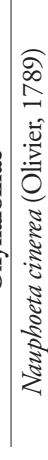 & 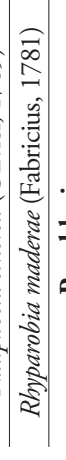 & 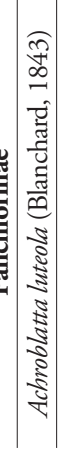 & 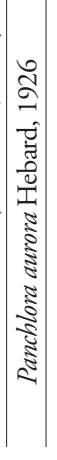 & 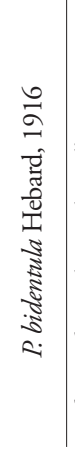 & 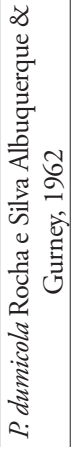 & 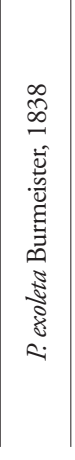 & 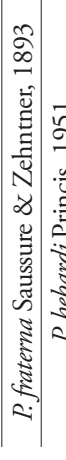 & 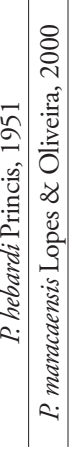 & 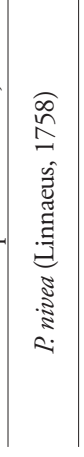 & 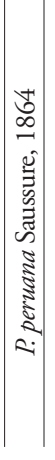 & 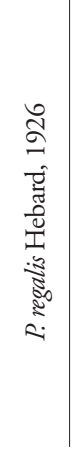 & 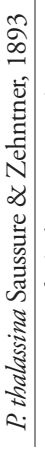 & 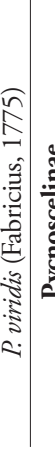 & 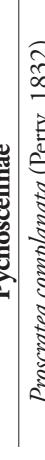 \\
\hline
\end{tabular}




\begin{tabular}{|c|c|c|c|c|c|c|c|c|c|c|c|c|c|c|c|c|c|c|c|c|c|}
\hline ڤ્ڤ & 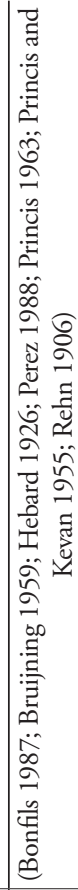 & & 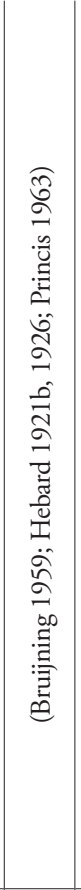 & 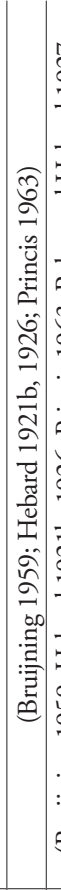 & 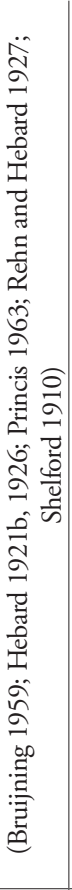 & 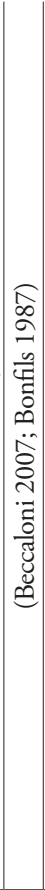 & 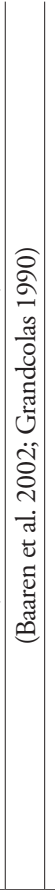 & 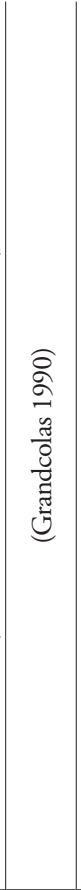 & 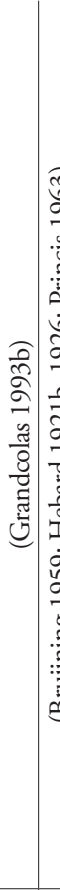 & 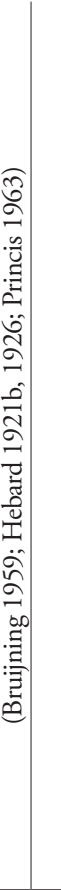 & & 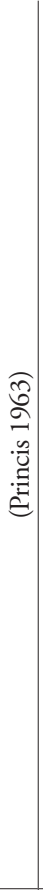 & 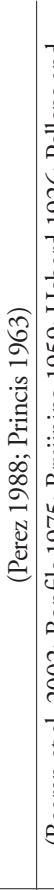 & 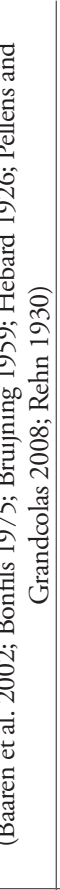 & 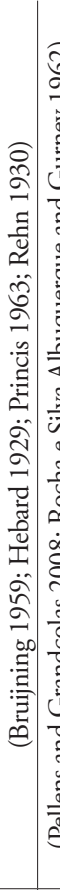 & 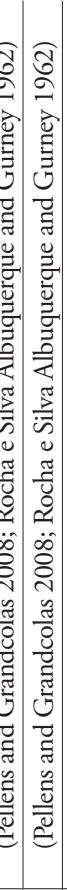 & 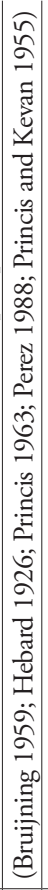 & 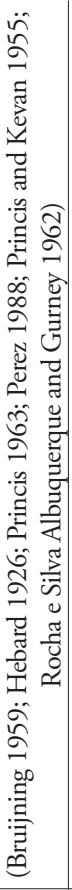 & 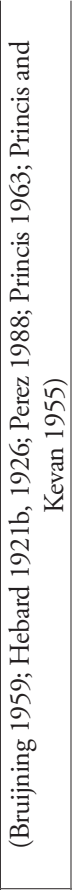 & & \\
\hline 촌 & $\circ$ & & & & & & & & & & & 0 & $\circ$ & 0 & & 0 & 0 & 0 & 0 & & \\
\hline v & $\circ$ & & 0 & 0 & $\circ$ & & 0 & $\circ$ & $\circ$ & 0 & & ० & 0 & $\circ$ & & & 0 & $\circ$ & $\circ$ & & $\circ$ \\
\hline క & $\circ$ & & 0 & 0 & $\circ$ & & & & & 0 & & 0 & 0 & $\circ$ & 0 & & 0 & 0 & 0 & & $\circ$ \\
\hline క్ర & $\circ$ & & $\circ$ & & & & & + & & & & 0 & 0 & & 0 & & 0 & 0 & $\circ$ & & 0 \\
\hline : & $\circ$ & & & & & & & & & & & 0 & 0 & & & & $\circ$ & $\circ$ & 0 & & \\
\hline จั & 0 & & & & & & & & & & & 0 & $\circ$ & & & & 0 & ० & 0 & & \\
\hline 今 & 0 & & & & & & & & & & & 0 & 0 & & & & 0 & 0 & 0 & & \\
\hline 논 & $\circ$ & & & & & 0 & & & & & & 0 & 0 & & & & 0 & 0 & 0 & & \\
\hline 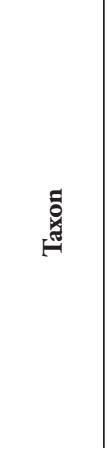 & 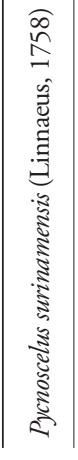 & 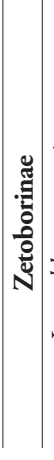 & 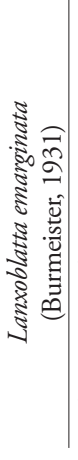 & 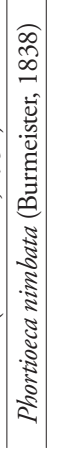 & 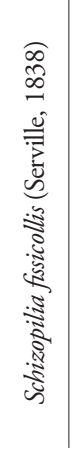 & 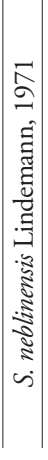 & 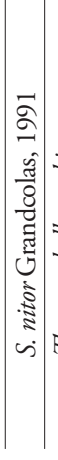 & 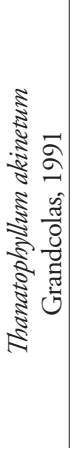 & 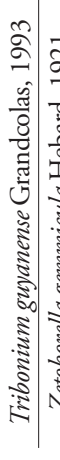 & 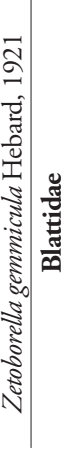 & 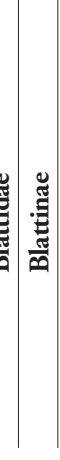 & 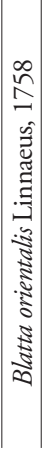 & 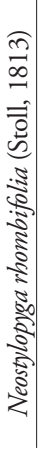 & 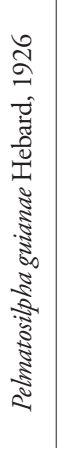 & 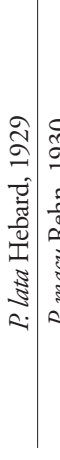 & 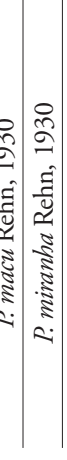 & 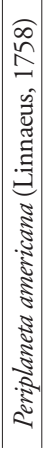 & 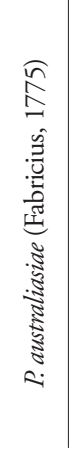 & 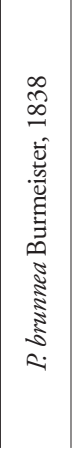 & (1) & 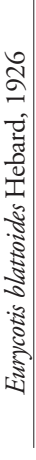 \\
\hline
\end{tabular}




\begin{tabular}{|c|c|c|c|c|c|c|c|c|c|c|c|c|c|c|c|c|c|c|c|c|}
\hline $\begin{array}{l}\mathscr{E} \\
\dot{\Xi}\end{array}$ & & 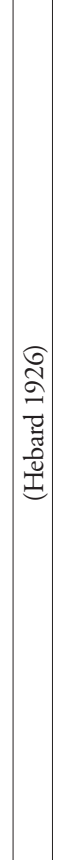 & & 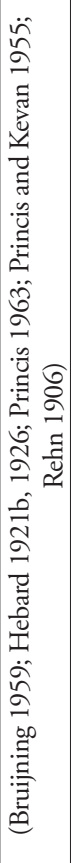 & & 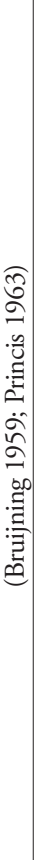 & 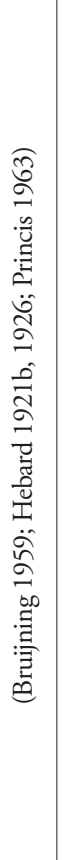 & 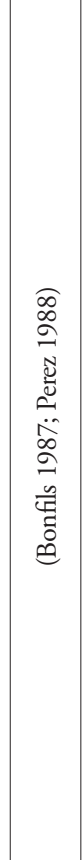 & 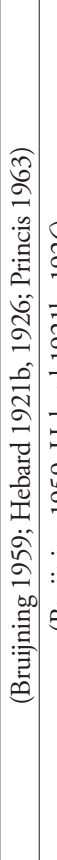 & 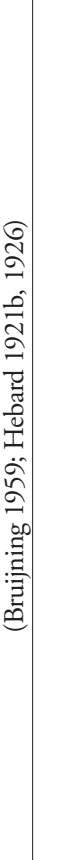 & & 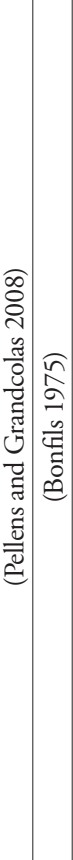 & 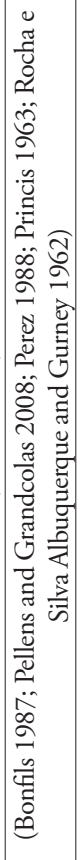 & 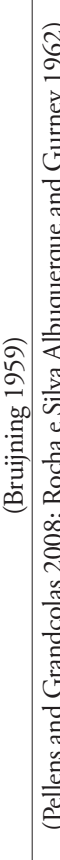 & 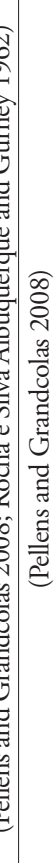 & 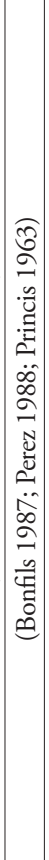 & 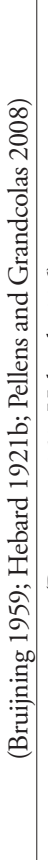 & 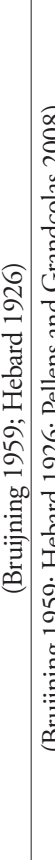 & 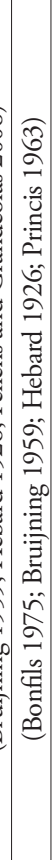 & 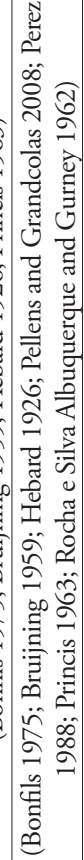 \\
\hline 촌 & & & & $\circ$ & & & n. & & & & & 0 & $\circ$ & & $\circ$ & & 0 & 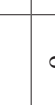 & b & \\
\hline v & & 0 & & 0 & & & 0 & & 0 & 0 & & 0 & & & & & 0 & 0 & 0 & $p$ \\
\hline క్ర & & 0 & & 0 & & 0 & 0 & & 0 & & & & & - & & & $\circ$ & 0 & 0 & \\
\hline S & & & & 0 & & & $n$. & & & & & & & & & & & 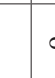 & & \\
\hline ఏ్ & & & & 0 & & & $n$. & & & & & & & & & & & & & \\
\hline จั & & & & $\circ$ & & & $n$. & & & & & & & & & & & & & \\
\hline 密 & & & & 0 & & & n. & & & & & & & & & & & & & \\
\hline 논 & & & & $\circ$ & & & n. & 0 & & & & & 0 & & & 0 & & & & 0 \\
\hline 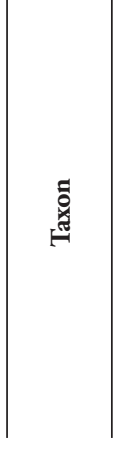 & ن & 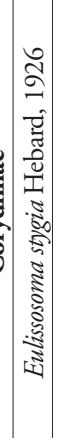 & 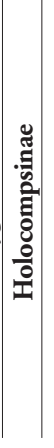 & 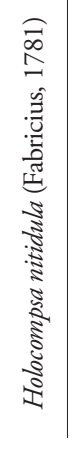 & : & 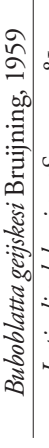 & 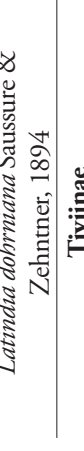 & 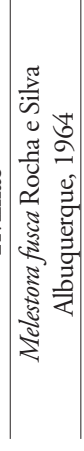 & 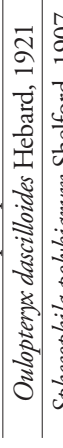 & 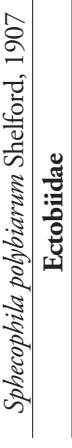 & . & 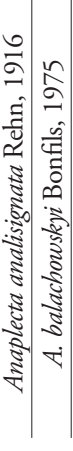 & 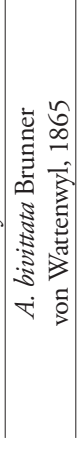 & 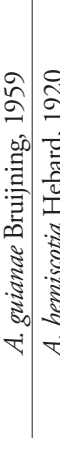 & 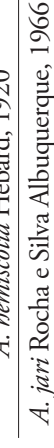 & 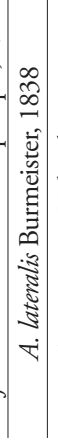 & 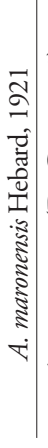 & 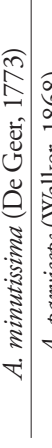 & 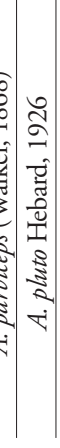 & 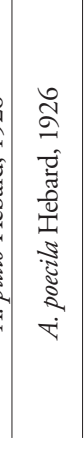 \\
\hline
\end{tabular}




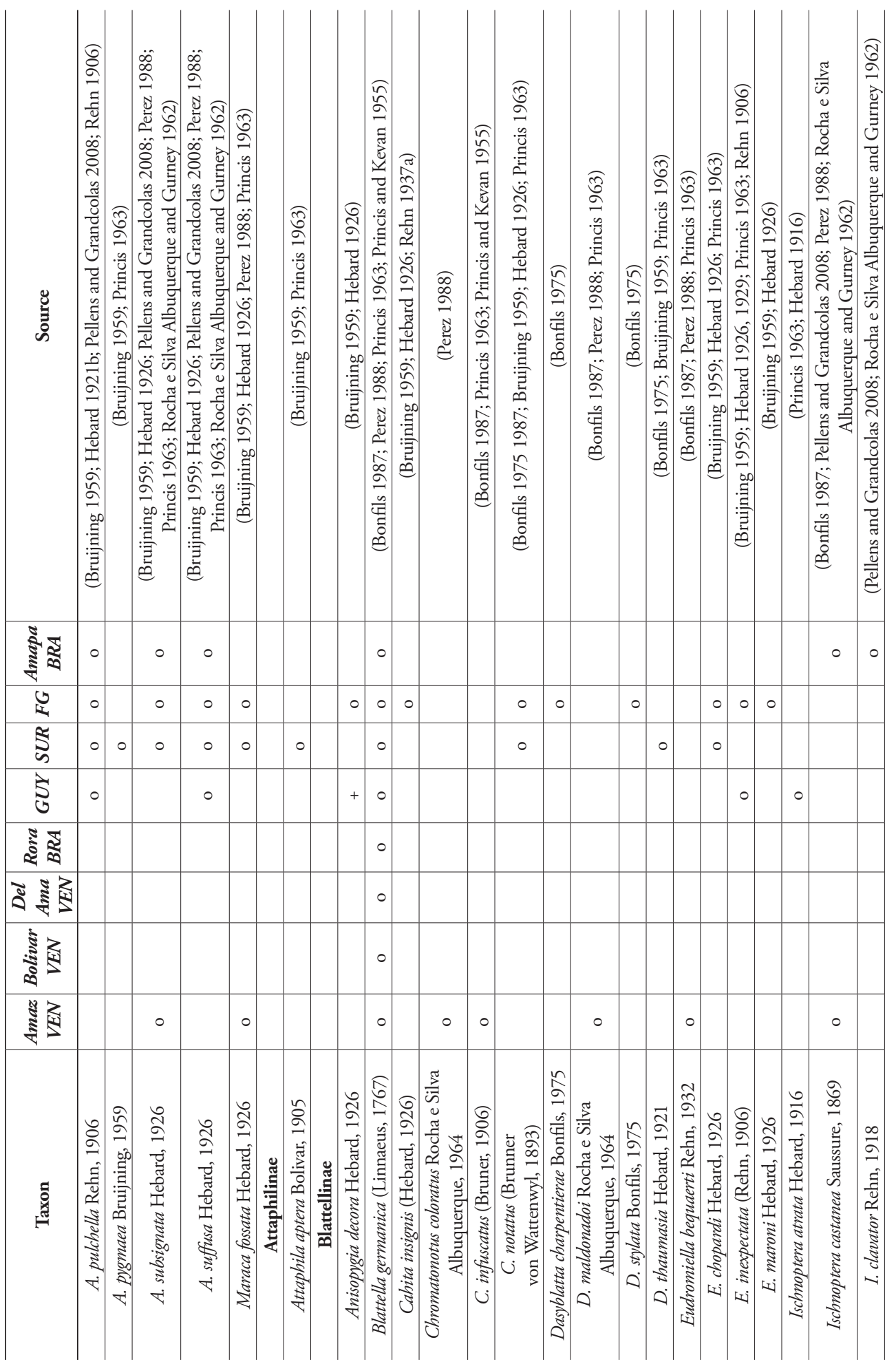




\begin{tabular}{|c|c|c|c|c|c|c|c|c|c|c|c|c|c|c|c|c|c|c|c|c|c|c|c|}
\hline ڤั & 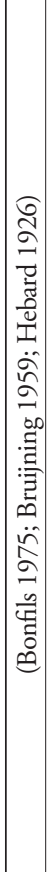 & 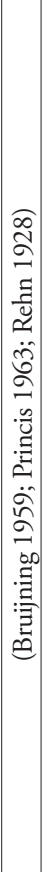 & 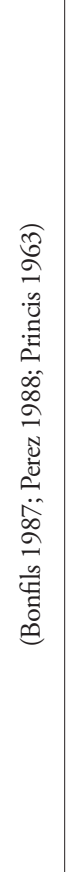 & 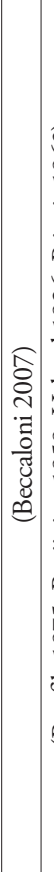 & 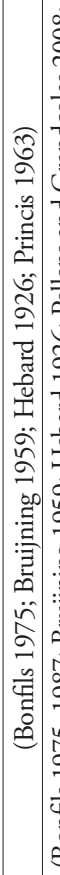 & 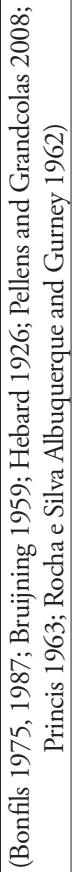 & 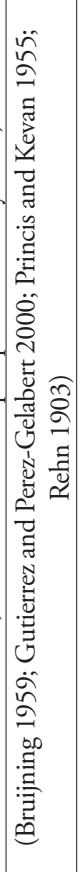 & 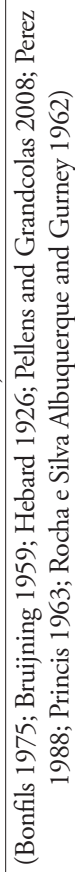 & 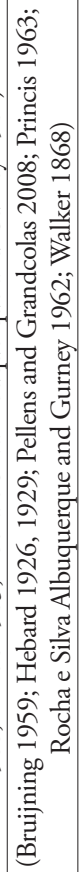 & 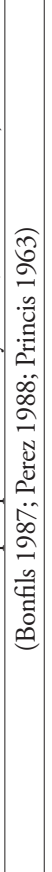 & 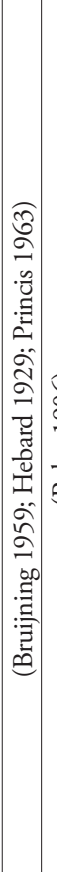 & 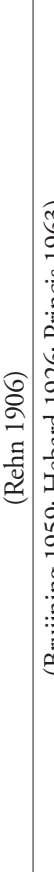 & 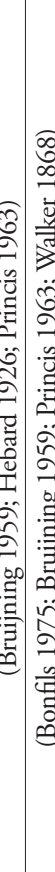 & 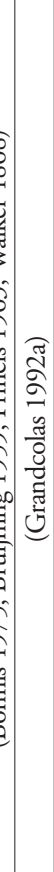 & 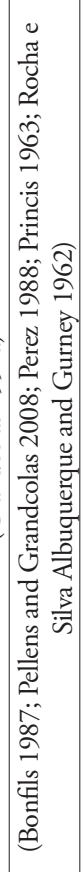 & 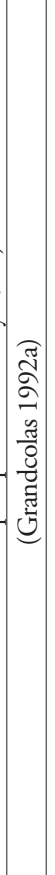 & 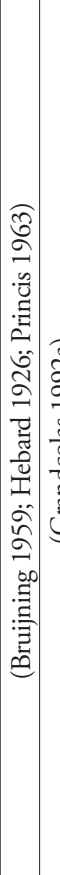 & & 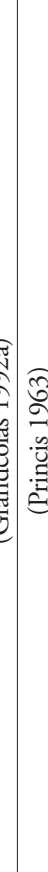 & 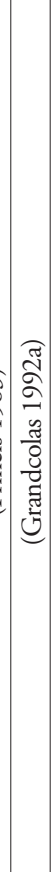 & 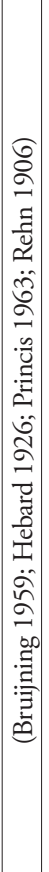 & : & 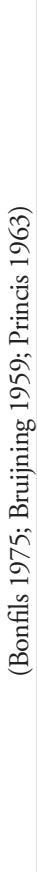 \\
\hline 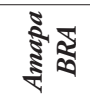 & & & & & & 0 & & 0 & 0 & & & & & & $\circ$ & & & & & & & & \\
\hline 诖 & 0 & & & 0 & 0 & $\circ$ & & 0 & $\circ$ & & & & $\circ 0$ & 0 & & $\circ$ & 0 & 0 & $\circ$ & 0 & 0 & & $\circ$ \\
\hline క్ & 0 & 0 & & & 0 & 0 & 0 & $\circ$ & $\circ$ & & $\circ$ & & 0 & & & & 0 & & & & & & 0 \\
\hline Ș & & $\circ$ & & & 0 & $\circ$ & $\circ$ & 0 & 0 & & 0 & 0 & 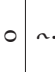 & + & & & & & $\circ$ & & 0 & + & \\
\hline : & & & & & & & & & & & & & & & & & & & & & & & \\
\hline จั & & & & & & & & & & & & & & & & & & & & & & & \\
\hline 离 & & & & & & & & & & & & & & & & & & & & & & & \\
\hline 논 & & & $\circ$ & & & & & 0 & & 0 & & & & & 0 & & & & & & & & \\
\hline 㺼 & 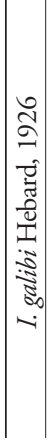 & 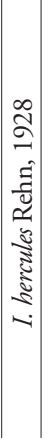 & 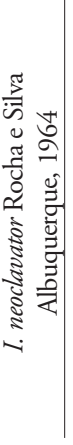 & 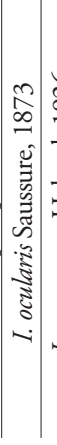 & 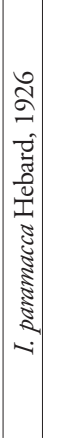 & 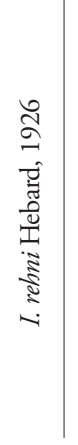 & 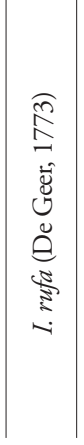 & 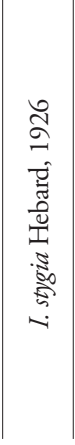 & 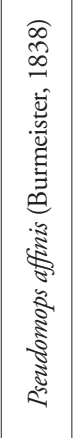 & 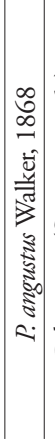 & 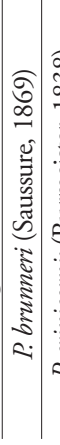 & 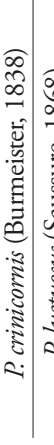 & 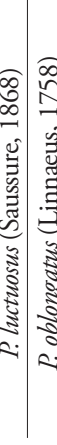 & 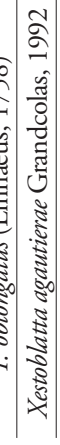 & 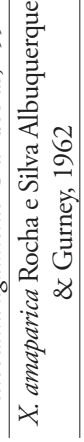 & 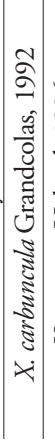 & 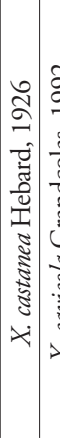 & 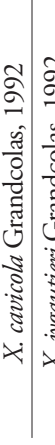 & 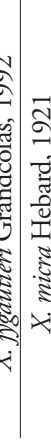 & 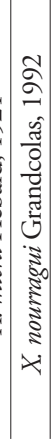 & 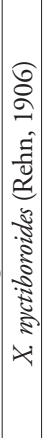 & 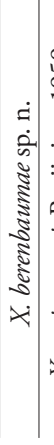 & 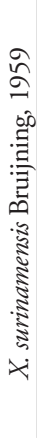 \\
\hline
\end{tabular}




\begin{tabular}{|c|c|c|c|c|c|c|c|c|c|c|c|c|c|c|c|c|c|c|c|c|c|}
\hline 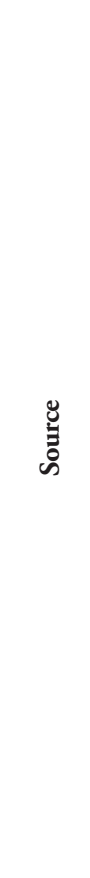 & & 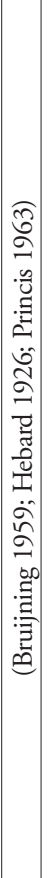 & 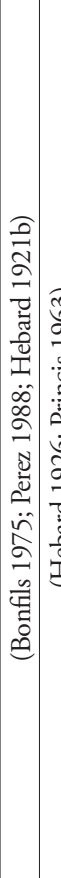 & 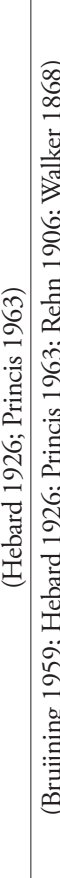 & 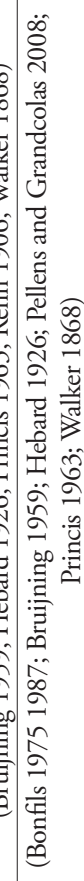 & 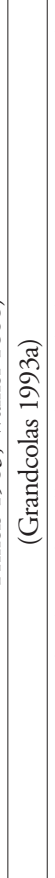 & 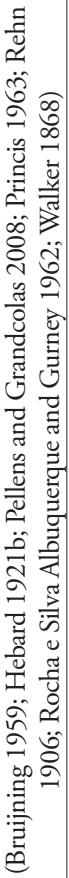 & 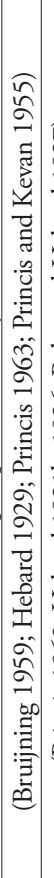 & 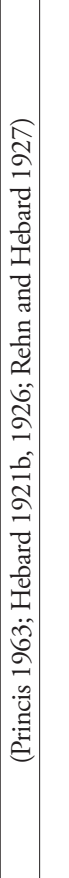 & 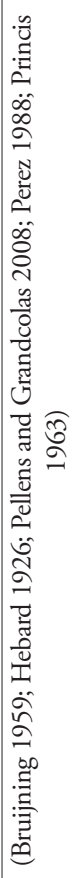 & 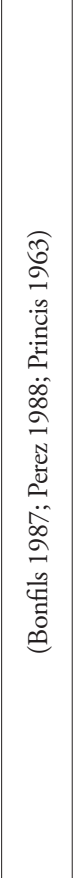 & 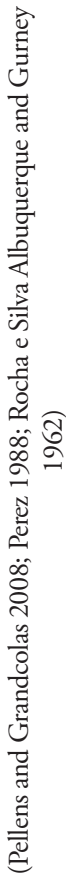 & 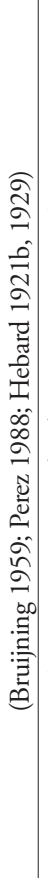 & 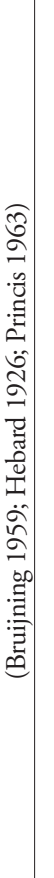 & 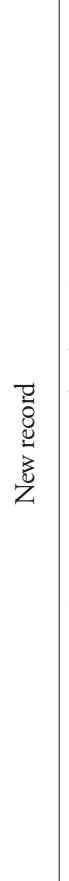 & 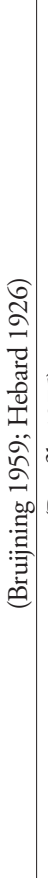 & & \begin{tabular}{l}
0 \\
\multirow{2}{0}{} \\
0 \\
0 \\
0 \\
0 \\
0
\end{tabular} & 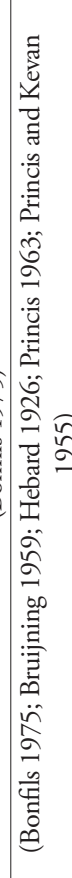 & 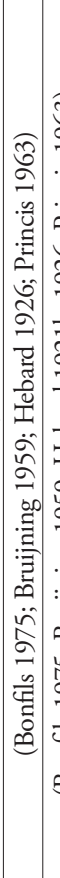 & 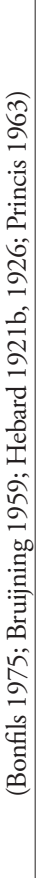 \\
\hline ș & & & & & $\circ$ & & 0 & & & $\circ$ & & $\circ$ & & & & & & & & & \\
\hline 证 & & $\circ$ & $\begin{array}{ll}0 & 0\end{array}$ & 0 & 0 & 0 & 0 & 0 & 0 & 0 & & & 0 & 0 & & 0 & $\circ$ & 0 & $\circ$ & 0 & $\circ$ \\
\hline $\begin{array}{l}5 \\
5 \\
5\end{array}$ & & 0 & & 0 & $\circ$ & & 0 & 0 & 0 & $\circ$ & & & 0 & & & & & & $\circ$ & 0 & 0 \\
\hline Ș & & & & +0 & $\circ$ & & $\circ$ & $\circ$ & & 0 & & & 0 & 0 & + & & & & & & $\circ$ \\
\hline 范 & & & & & & & & & & & & & & & & & & & & & \\
\hline จั & & & & & & & & & & & & & & & & & & & & & \\
\hline 离 & & & & & & & & & & & & & & & & & & & & & \\
\hline 논 & & & 0 & & & & & & & $\circ$ & 0 & $\circ$ & & & & & & & & & \\
\hline 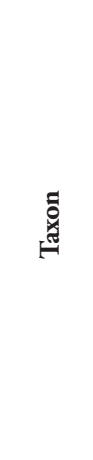 & 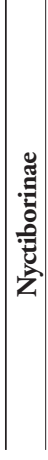 & 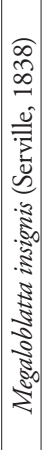 & 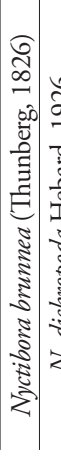 & 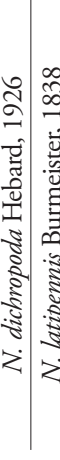 & 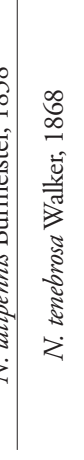 & 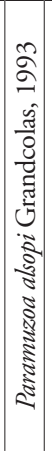 & 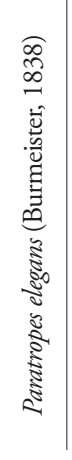 & 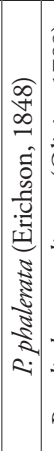 & 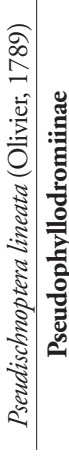 & 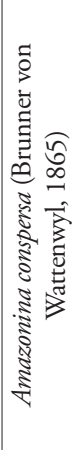 & 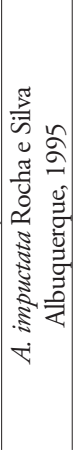 & 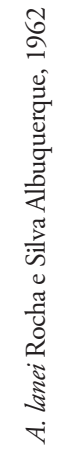 & 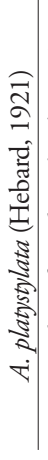 & 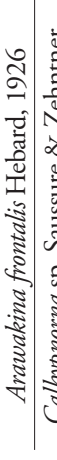 & 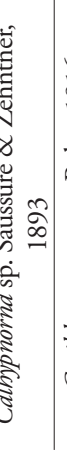 & 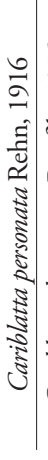 & 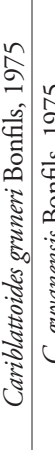 & 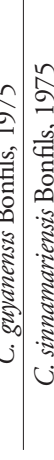 & 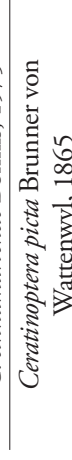 & 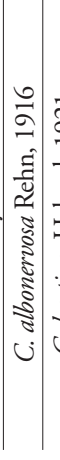 & 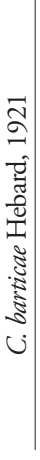 \\
\hline
\end{tabular}




\begin{tabular}{|c|c|c|c|c|c|c|c|c|c|c|c|c|c|c|c|c|c|c|c|c|c|c|c|c|}
\hline ڤ્ & 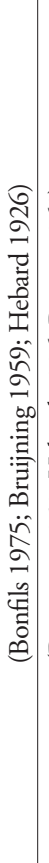 & 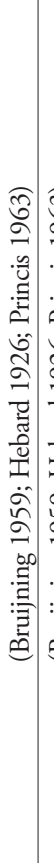 & 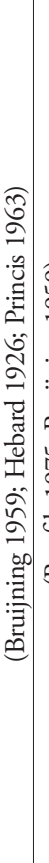 & 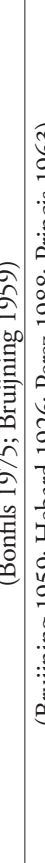 & 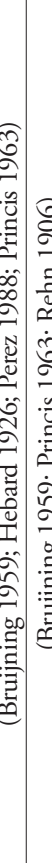 & 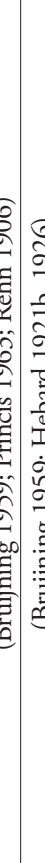 & 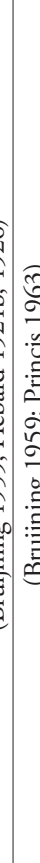 & 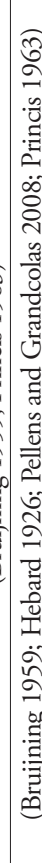 & 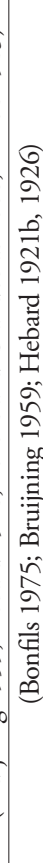 & 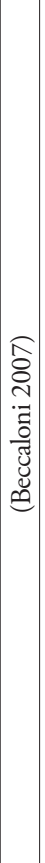 & 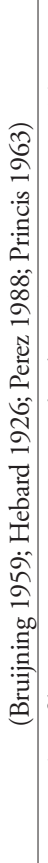 & 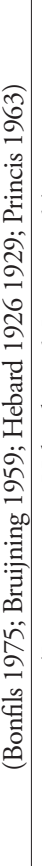 & 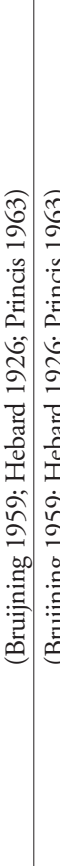 & 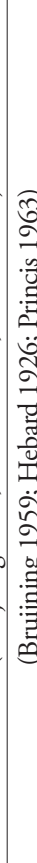 & 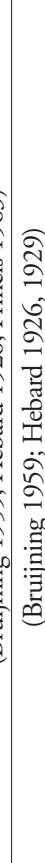 & 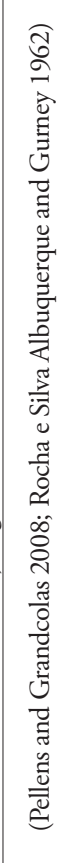 & 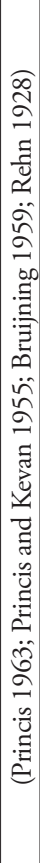 & 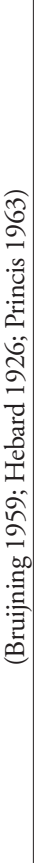 & 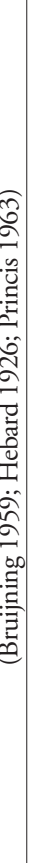 & 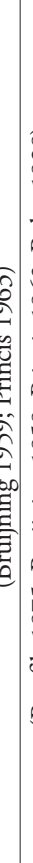 & 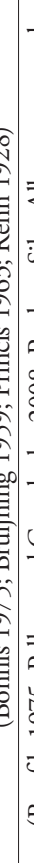 & 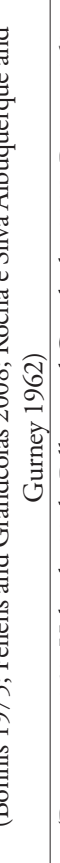 & 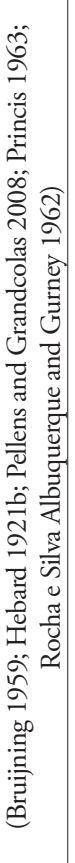 & 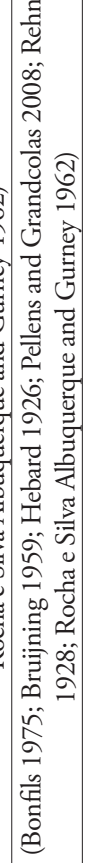 \\
\hline 起 & & & & & & & & $\circ$ & & & & & & & & 0 & & & & & & 0 & & \\
\hline 足 & 0 & 0 & $\circ$ & 0 & 0 & 0 & & $\circ$ & $\circ$ & 0 & 0 & 0 & $\circ$ & 0 & 0 & & & 0 & 0 & & 0 & $\circ$ & 0 & \\
\hline 5 & 0 & 0 & 0 & 0 & 00 & 0 & $\circ$ & 0 & $\circ$ & & 0 & 0 & 0 & 0 & 0 & & 0 & 0 & $\circ$ & 0 & 0 & & & \\
\hline క్ర & & & & & 0 & 0 & & $\circ$ & & & 0 & 0 & $\circ$ & & $\circ$ & & 0 & & & & 0 & & 0 & \\
\hline 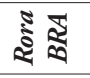 & & & & & & & & & & & & & & & & & & & & & & & 0 & \\
\hline ฐ ฐ & & & & & & & & & & & & & & & & & & & & & & & & \\
\hline : & & & & & & & & & & & & & & & & & & & & & & & & \\
\hline 资过 & & & & & & & & & & & & & & & & & & & & & & & & \\
\hline 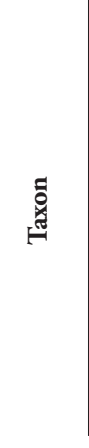 & 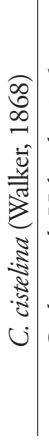 & 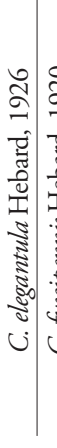 & 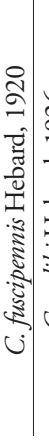 & 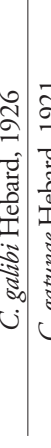 & 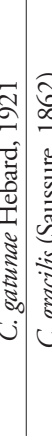 & 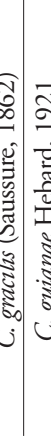 & 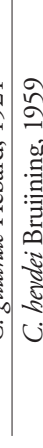 & 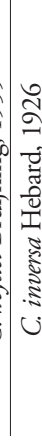 & 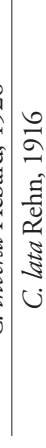 & 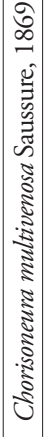 & 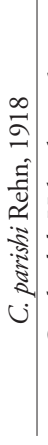 & 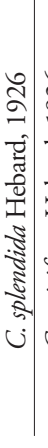 & 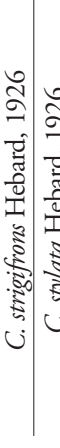 & $\begin{array}{l}\infty \\
0 \\
\infty \\
0 \\
0 \\
0 \\
= \\
0 \\
\text { है } \\
\text { है } \\
\text { है }\end{array}$ & 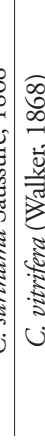 & 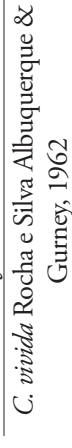 & 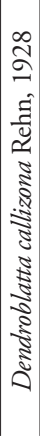 & 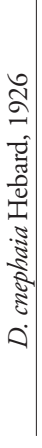 & 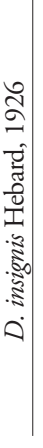 & 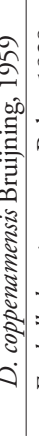 & בֶّ & 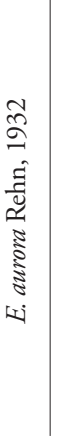 & 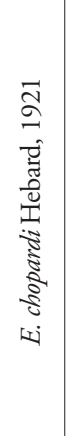 & 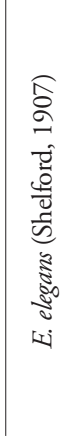 \\
\hline
\end{tabular}




\begin{tabular}{|c|c|c|c|c|c|c|c|c|c|c|c|c|c|c|c|c|c|c|c|c|c|c|}
\hline ڤั & 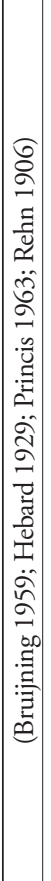 & 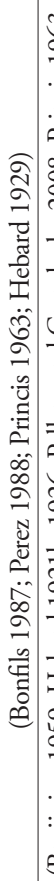 & 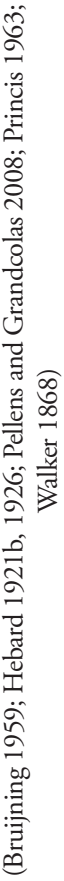 & 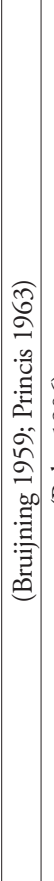 & 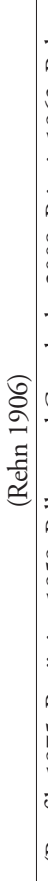 & 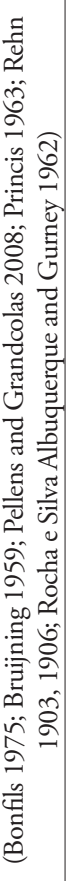 & 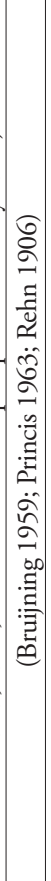 & 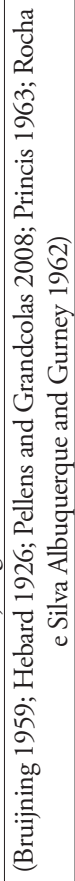 & 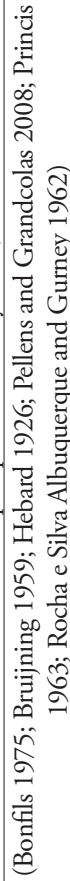 & 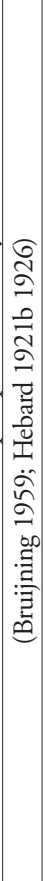 & 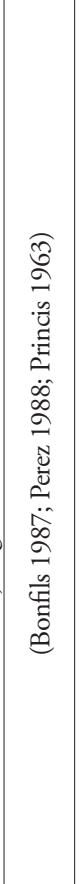 & 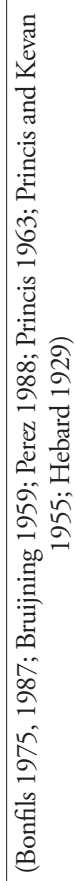 & 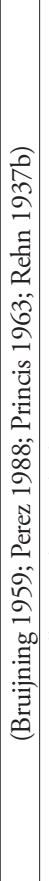 & 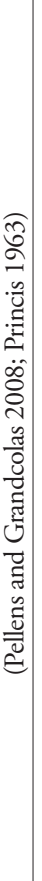 & 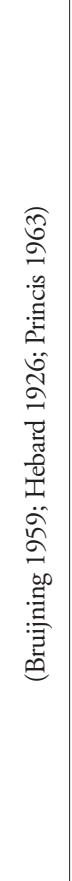 & 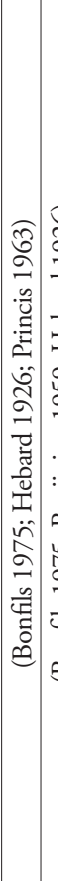 & 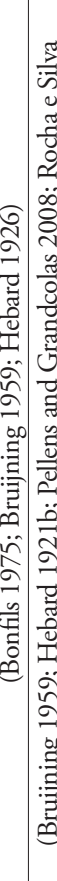 & 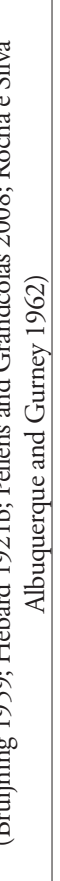 & 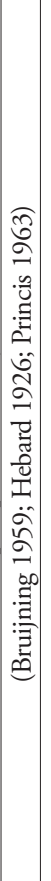 & 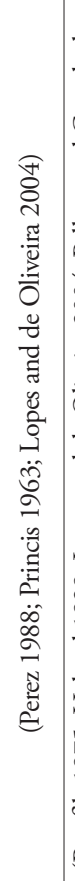 & 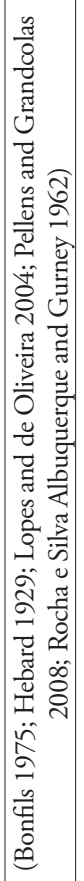 & 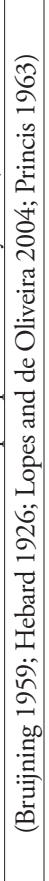 \\
\hline 논 & & & $\circ$ & & & 0 & & $\circ$ & 0 & & & & & 0 & & & & $\circ$ & & & & \\
\hline 诖 & & & 0 & & & 0 & & 0 & 0 & 0 & & & & $\circ$ & $\circ$ & 0 & 0 & & 0 & & 0 & ○ \\
\hline క & 0 & & ० & 0 & & 0 & & 0 & ० & & & $\circ$ & 0 & 0 & 0 & $\circ$ & & & 0 & & & 0 \\
\hline S & 0 & & $\circ$ & & 0 & $\circ$ & 0 & & & & & $\circ$ & 0 & & 0 & & & & & & $\circ$ & \\
\hline 竎 & & & & & & & & & & & & & & & & & & & & & & \\
\hline ฉัฐ & & n. & & & & & & & & & & & & & & & & & & & & \\
\hline 离 & & $n$. & & & & & & & & & & & & & & & & & & & & \\
\hline 논 & & n. & & & & & & & & & 0 & & 0 & & & & & & & 0 & & \\
\hline 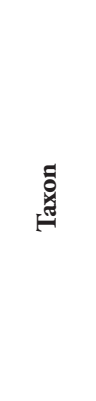 & 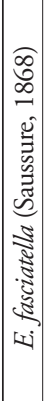 & 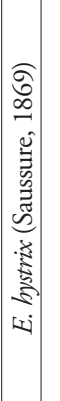 & 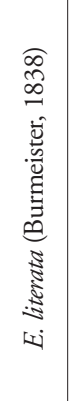 & 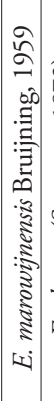 & 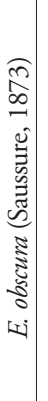 & 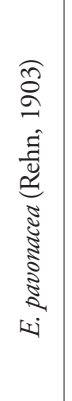 & 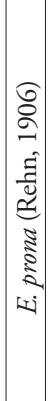 & 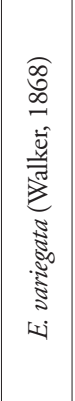 & 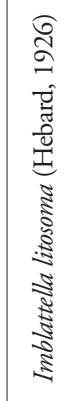 & 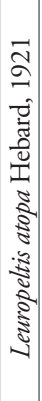 & 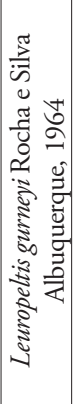 & 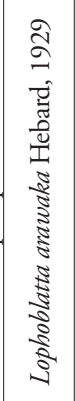 & 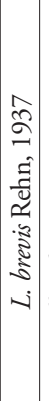 & 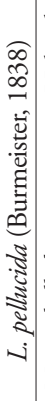 & 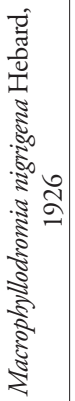 & 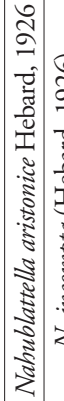 & 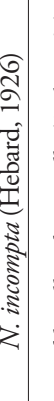 & 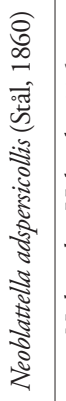 & 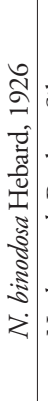 & 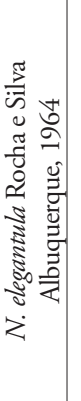 & 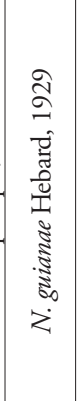 & 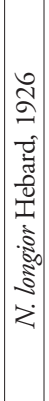 \\
\hline
\end{tabular}




\begin{tabular}{|c|c|c|c|c|c|c|c|c|c|c|c|c|c|c|c|c|c|c|c|c|}
\hline $\begin{array}{l}8 \\
\dot{\Xi}\end{array}$ & 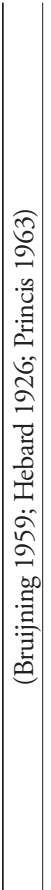 & 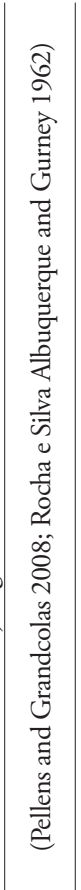 & 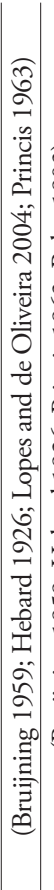 & 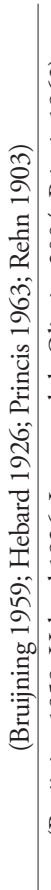 & 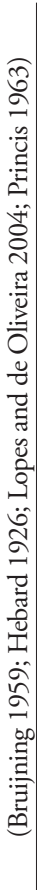 & 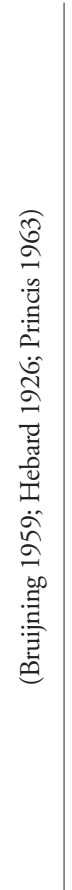 & 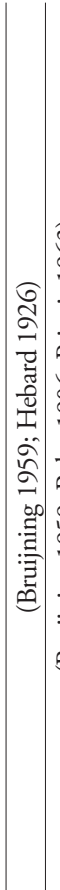 & 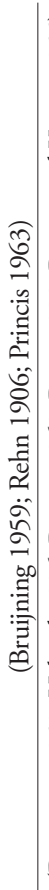 & 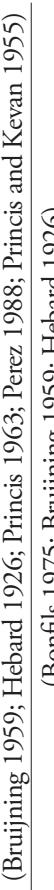 & 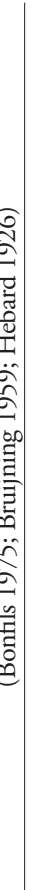 & 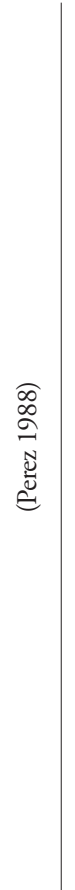 & 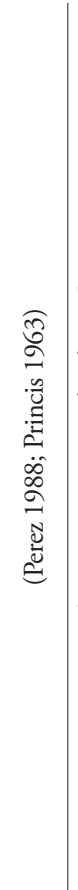 & 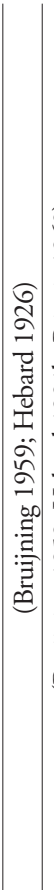 & 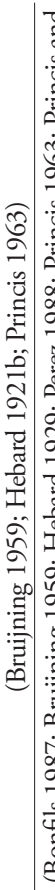 & 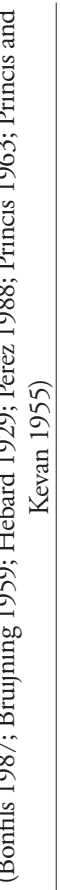 & 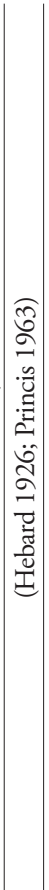 & 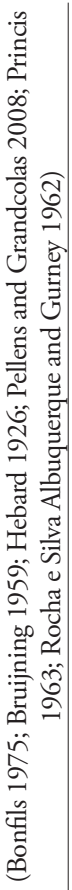 & & 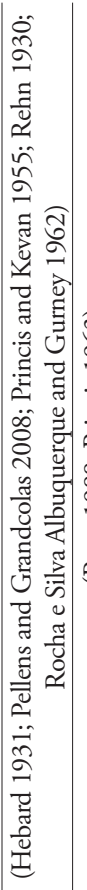 & 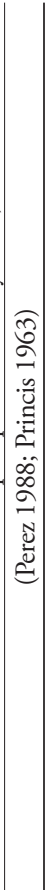 \\
\hline 촌 & & 0 & & & & & & & & & & & & & 0 & & $\circ$ & & $\circ$ & \\
\hline v & 0 & & 0 & 0 & 0 & $\circ$ & 0 & & 0 & 0 & & & 0 & 0 & 0 & 0 & 0 & & & \\
\hline S & 0 & & 0 & 0 & & 0 & 0 & 0 & 0 & 0 & & & & & ० & 0 & & & & \\
\hline Ș & & & & 0 & & & & 0 & 0 & & & & & 0 & ० & & 0 & & & \\
\hline కัฐ & & & & & & & & & & & & & & & 0 & & & & & \\
\hline จั & & & & & & & & & & & & & & & 0 & & & & & \\
\hline 离 & & & & & & & & & & & & & & & $\circ$ & & & & & \\
\hline 닌 & & & & & & & & & 0 & & 0 & $\circ$ & & & 0 & & & & & \\
\hline 㺼 & 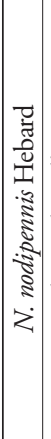 & 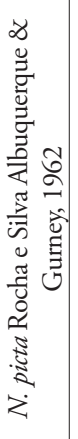 & 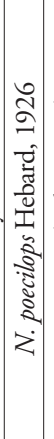 & 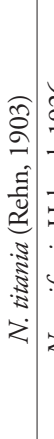 & 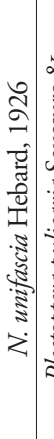 & 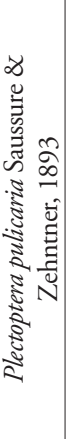 & 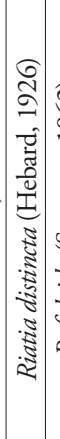 & 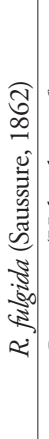 & 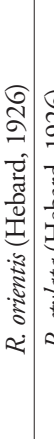 & 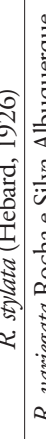 & 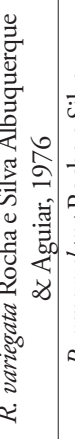 & 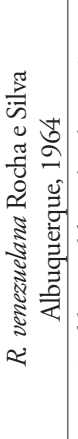 & 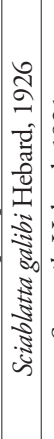 & 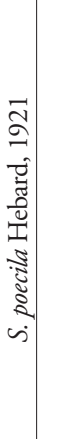 & 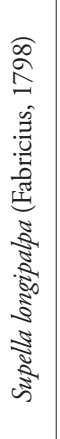 & 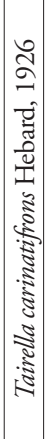 & 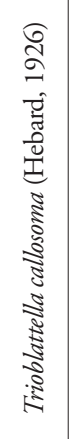 & 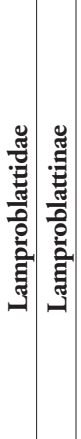 & 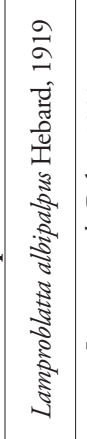 & 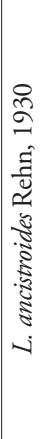 \\
\hline
\end{tabular}


意最量

言量

纪 莺

के

○ छ

苛密

1.

乙

它

岂

के

है

$\Xi$ ते

펑

\&

巳 일

苛

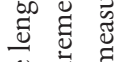

글

ญ ๕

ร 둰

娄

这

号

$\Xi 3$ के

चै

:

월

궐

के के

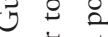

छํㅝㄴ

क के

प्ष

०

気 离

Чั

苛苛

ญ्ठ

วับ

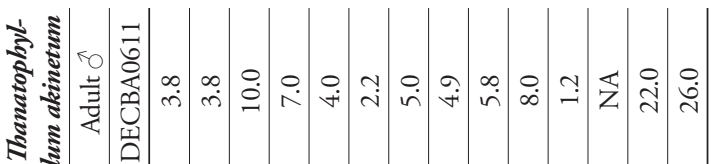

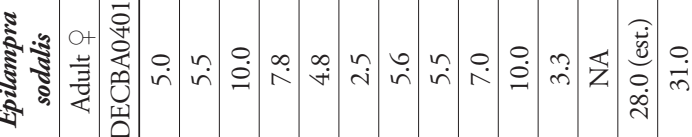

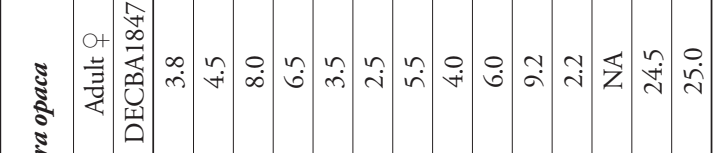

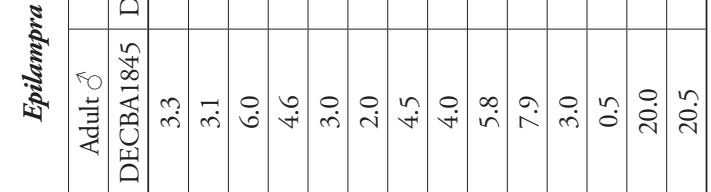

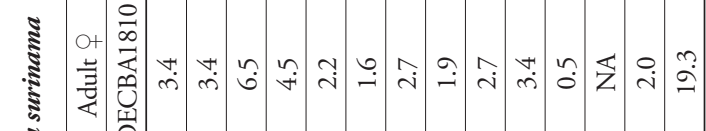

క్

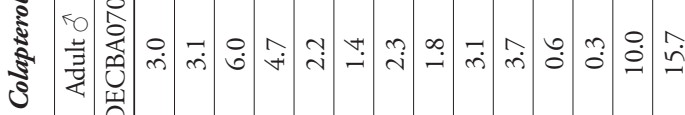

पे 싱

돈

च

을 त

《

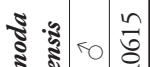

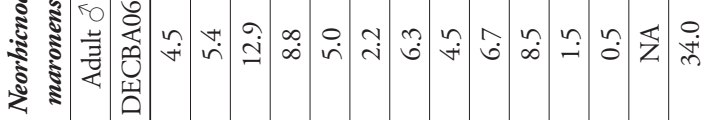
政

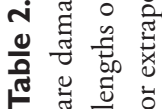

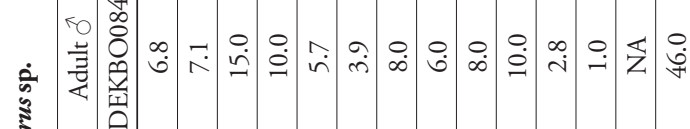

है

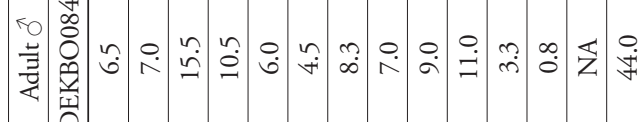

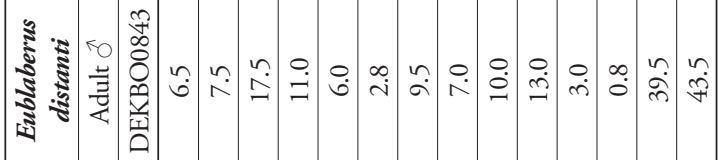

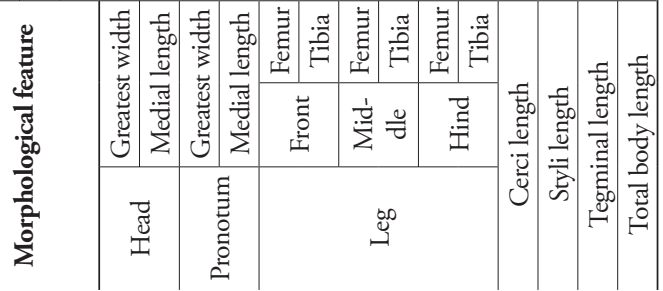




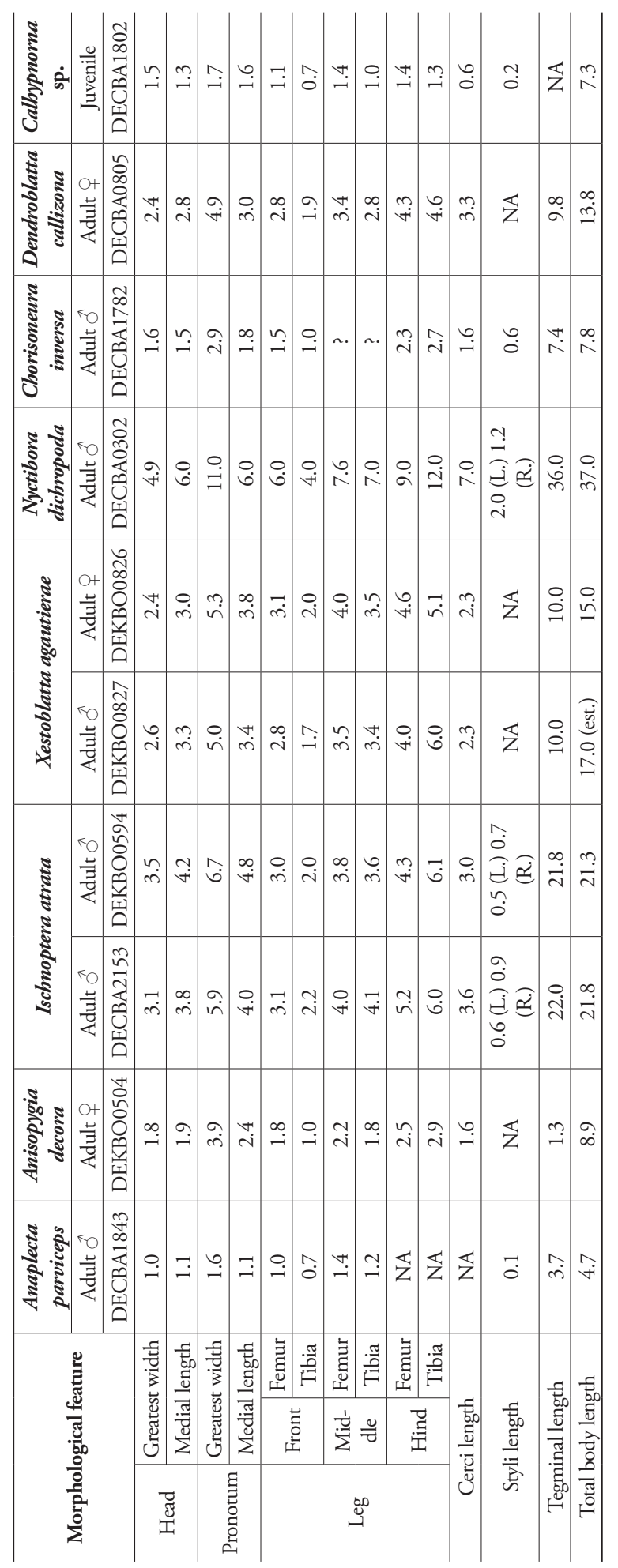




\section{Subfamily: Blaberinae}

Eublaberus distanti (Kirby, 1903)

Materials. Adult $\widehat{\partial}$.

Voucher number: DEKBO0843.

Collection locale. Karanambu Ranch, Rupununi, Guyana.

GPS: $3^{\circ} 45^{\prime} 2.2^{\prime \prime} \mathrm{N}, 59^{\circ} 18^{\prime} 31.2^{\prime \prime} \mathrm{W}$.

Date: 7 - June - 2013.

Collectors. Dominic A. Evangelista, Oswin Ambrose, Susan George, and Megan M. Wilson.

Collection/ecological information. This specimen was collected in the bathroom of one of the cabins at the camp of Karanambu Ranch.

Known geographic distribution. Guatemala, Costa Rica, Panama, Colombia, Trinidad and Tobago, French Guiana, Suriname, Guyana and Brazil

\section{Neorbicnoda maronensis (Hebard, 1921)}

Materials. Adult $\widehat{\jmath}$ Figure 1.

Voucher number: DECBA0615.

GenBank accession number: KF155090.

Collection locale. CEIBA Biological Station, Madewini, Guyana.

GPS: $6^{\circ} 29^{\prime} \mathrm{N}, 58^{\circ} 13^{\prime} \mathrm{W}$.

Date: 02 - January - 2012 .

Collectors. Dominic A. Evangelista, Ian Biazzo, Joseph A. Evangelista, Paul Frandsen, William R. Kuhn, and Jessica L. Ware.

Collection/ecological information. This specimen was caught in a pitfall trap baited with beer in an uplands secondary forest.

Morphological identification. This specimen agrees with the description of the male genitalia in Grandcolas (1992b).

Known geographic distribution. Guyana (new record), Suriname, and French Guiana.

\section{Subfamily: Epilamprinae}

Colapteroblatta surinama (Saussure, 1868)

Materials. Adult $\widehat{~}$ Figure 2E.

Voucher number: DECBA0703.

GenBank accession number: KF155029.

Collection locale. CEIBA Biological Station, Madewini, Guyana. 


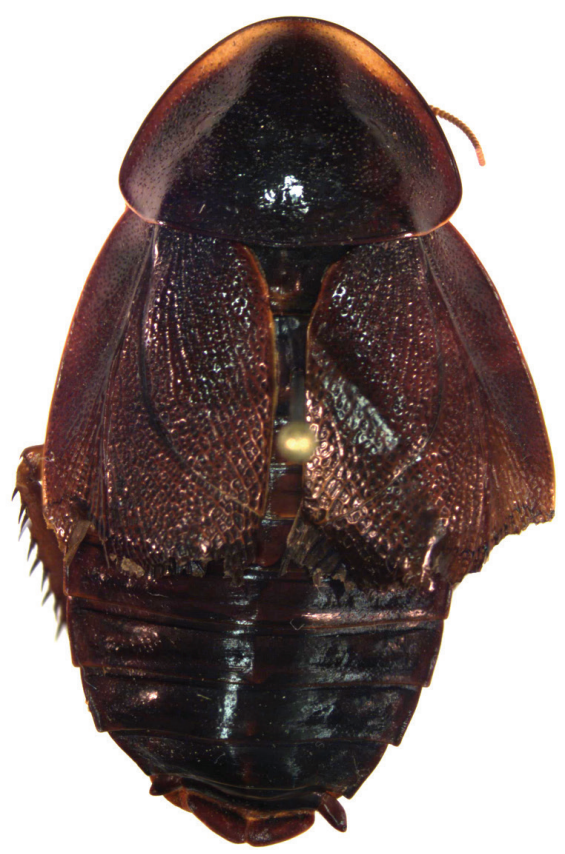

Figure I. Neorhicnoda maronensis adult male (DECBA0615).

GPS: $6^{\circ} 29^{\prime} \mathrm{N}, 58^{\circ} 13^{\prime} \mathrm{W}$.

Date: 05 - August - 2011.

Collectors. Dominic A. Evangelista, Ian Biazzo, Manpreet K. Kohli, Melissa Sanchez-Herrera, Nicole Sroczinski, and Jessica L. Ware.

Collection/ecological information. This specimen was collected in an uplands secondary forest from within a rotting vine.

Morphological identification. This specimen was identified using Roth and Gutierrez (1998).

Adult + Figure 2D.

Voucher number: DECBA1810.

GenBank accession number: KF155126.

Collection locale. CEIBA Biological Station, Madewini, Guyana.

GPS: $6^{\circ} 29^{\prime} \mathrm{N}, 58^{\circ} 13^{\prime} \mathrm{W}$.

Date: 20 - August -2011.

Collectors. Dominic Evangelista and William R. Kuhn.

Collection/ecological information. This specimen was collected in an uplands secondary forest from within an arboreal bromeliad.

Morphological identification. This specimen was identified using Roth and Gutierrez (1998). 


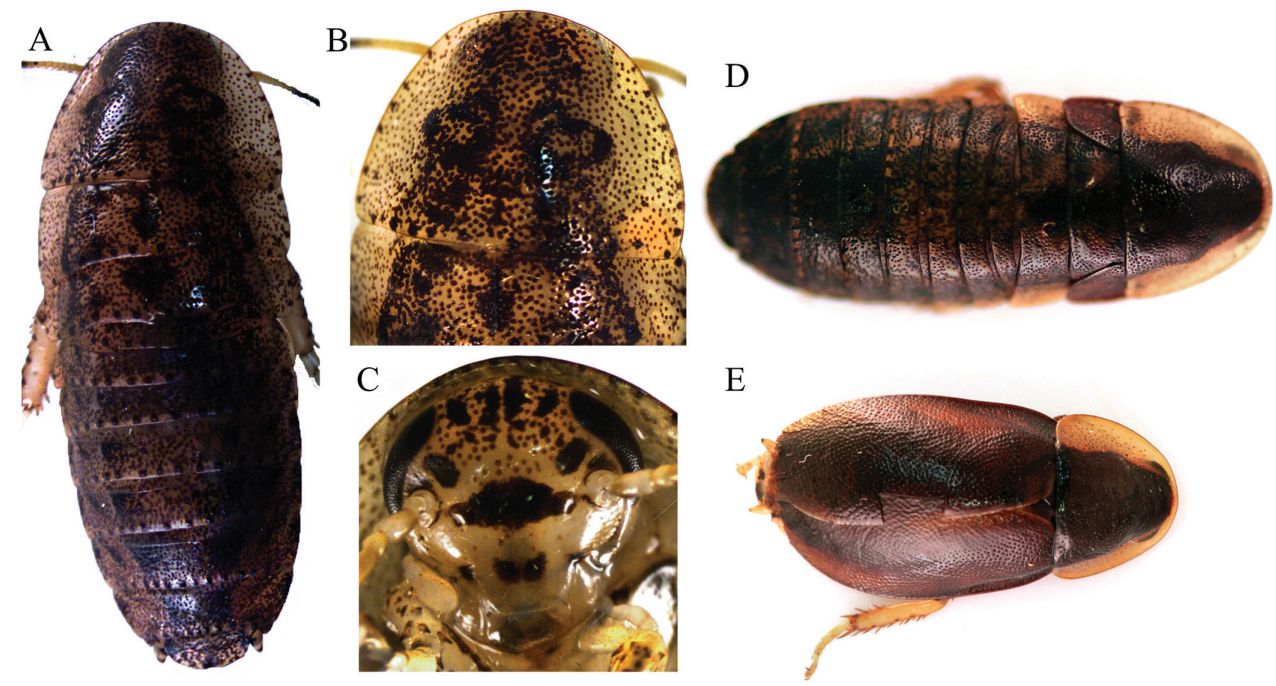

Figure 2. Colapteroblatta surinama. A-C Juvenile (dorsal aspect, pronotum, ventral aspect of head) D Adult female, dorsal aspect E Adult male, dorsal aspect. Photos not to scale.

Juvenile Figure 2A-C.

Voucher number: DECBA1811.

GenBank accession number: KF155112.

Collection locale. CEIBA Biological Station, Madewini, Guyana.

GPS: $6^{\circ} 29^{\prime} \mathrm{N}, 58^{\circ} 13^{\prime} \mathrm{W}$.

Date: 17 - August - 2013.

Collectors. Dominic Evangelista and William R. Kuhn.

Collection/ecological information. This specimen was collected on vegetation in an uplands secondary forest.

Morphological identification. This specimen was associated to its adult morph using barcodes in Evangelista et al. (2014). The overall coloration of the juvenile specimens of this species is more similar to that of $C$. darlingtoni Roth \& Gutiérrez, 1998 and $C$. rehni Roth \& Gutiérrez, 1998 than to that of the adults of its own species (see Figure 2).

Genetic information and evolutionary placement. All three specimens have nearly identical cytochrome oxidase I (COI) haplotypes but their position could not be determined relative to other cockroach species with the data evaluated by Evangelista et al. (2014).

Known geographic distribution. Guyana, Suriname.

\section{Epilampra opaca Walker, 1868}

Materials. Adult के Figure 3B. 

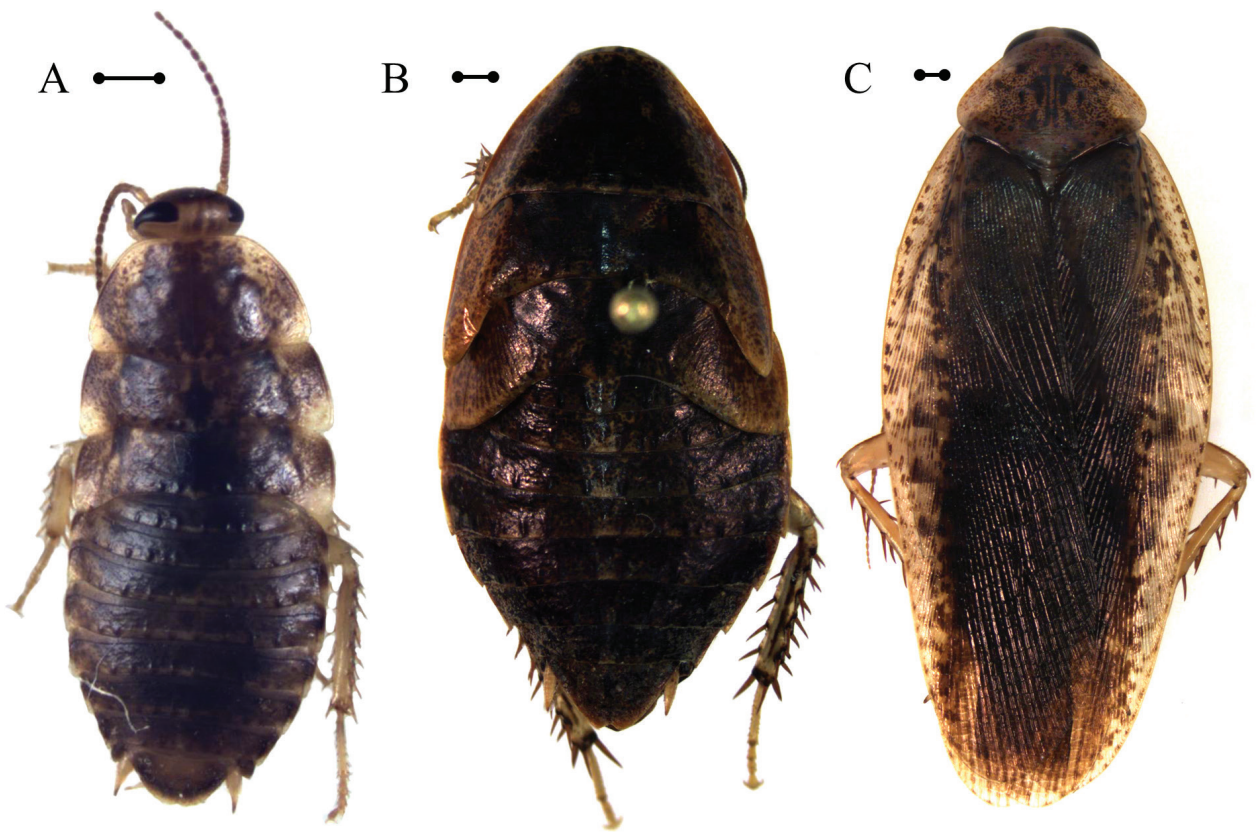

Figure 3. Epilampra opaca. A Early juvenile instar (DEDSM0141) B Late juvenile instar (DECBA1706) C Adult (DECBA1845). Scale bars approximate $1 \mathrm{~mm}$.

Voucher number: DECBA1845.

GenBank accession number: KF155125.

Collection locale. CEIBA Biological Station, Madewini, Guyana.

GPS: $6^{\circ} 29^{\prime} \mathrm{N}, 58^{\circ} 13^{\prime} \mathrm{W}$.

Date: 18 - August - 2012.

Collectors. Dominic A. Evangelista and William R. Kuhn.

\section{Adult 우}

Voucher number: DECBA1847.

GenBank accession number: KF155124.

Collection locale. CEIBA Biological Station, Madewini, Guyana.

GPS: $6^{\circ} 29^{\prime \prime N}, 58^{\circ} 13^{\prime \prime W}$.

Date: 5 - August - 2011 .

Collectors. Dominic A. Evangelista, Ian Biazzo, Manpreet K. Kohli, Melissa Sanchez-Herrera, Nicole Sroczinski and Jessica L. Ware.

Collection/ecological information. The adult male (DECBA1845) was collected at a light trap. Adult female (DECBA1847) was collected by hand in the leaf litter by a small pond. Most late instar individuals of this species were also collected at the edge of this pond and some were collected in pitfall traps baited with beer. Early instar individuals of this species were collected from within bromeliads. 
Genetic information. The two adult specimens reported here, as well as three juvenile individuals (Voucher and accession numbers: DEDSM0141 - KF155097, DECBA1706 - KF155089, DECBA0205 - KF155088) have identical COI barcodes and are sister to each other on the tree. However, other individuals (similar to E. opaca) included in the analysis (Voucher and accession numbers: DECBA0214 - KF155018, DECBA0216 - KF155017, DECBA0606 - KF155013, DECBA1101 - KF155016, DECBA0605 - KF155012, DECBA0608 - KF155015) are more genetically diverse and are only supported as monophyletic by $63 \%$ bootstrap support.

Morphological identification. There is a great deal of intraspecific variation in the morphology of this species. Early instar nymphs are difficult to associate to later instar nymphs, all of which are entirely unrecognizable from the adults (Figure 3A-C). Furthermore, there is variation within instars, where some later instar nymphs will appear to have a medially divided subgenital plate and others do not. This trait was not found to correlate with genetic differences (Evangelista et al. 2014).

The external morphology of this species provides little assistance in its identification, as most descriptions of it emphasize coloration that is both subtle and variable. However, the allometry of our specimens (Table 2) agree with those of Bruijning (1959). A definitive identification was made by comparison of genital morphology using Roth (1970b), particularly in the shape of the prepuce.

Known geographic distribution. Venezuela (unverified), Guyana, Suriname, French Guiana and Brazil

History and synonymy. Walker (1868) first described both E. opaca Walker, 1868 and E. substrigata Walker, 1868. Hebard (1926) noted that E. opaca Walker, 1868 has a highly variable morphology and may be synonymous with a few other $E p$ ilampra (e.g. E. conferta Walker, 1868 syn. stigmosa Giglio-Tos, 1898, E. maculicollis (Serville, 1838)). This variability is evident in the work published by Roth (1970b), which shows a great deal of variation in the genital morphology, in particular for L2d. Although it is not clear if anyone before Roth (1970b) examined the genitalia of these two species, both Shelford (1910) and Princis (1963) considered them to be synonyms. Roth's (1970b) photos show that, although each species is intraspecifically variable, both are distinct and separable by the shape of L2d and the prepuce. Roth himself acknowledged this and considered the species as being separate. Although we have not examined any E. substrigata Walker, 1868, we agree with Roth's interpretation of the morphology and follow from his precedence in considering these separate (see Roth $1970 \mathrm{~b}$ for the opinions of Princis and Gurney on the status of these two species).

\section{Epilampra sodalis Walker, 1868}

Materials. Adult §̂ Figure 4A.

Voucher number: DECBA0401.

GenBank accession number: KF155063. 

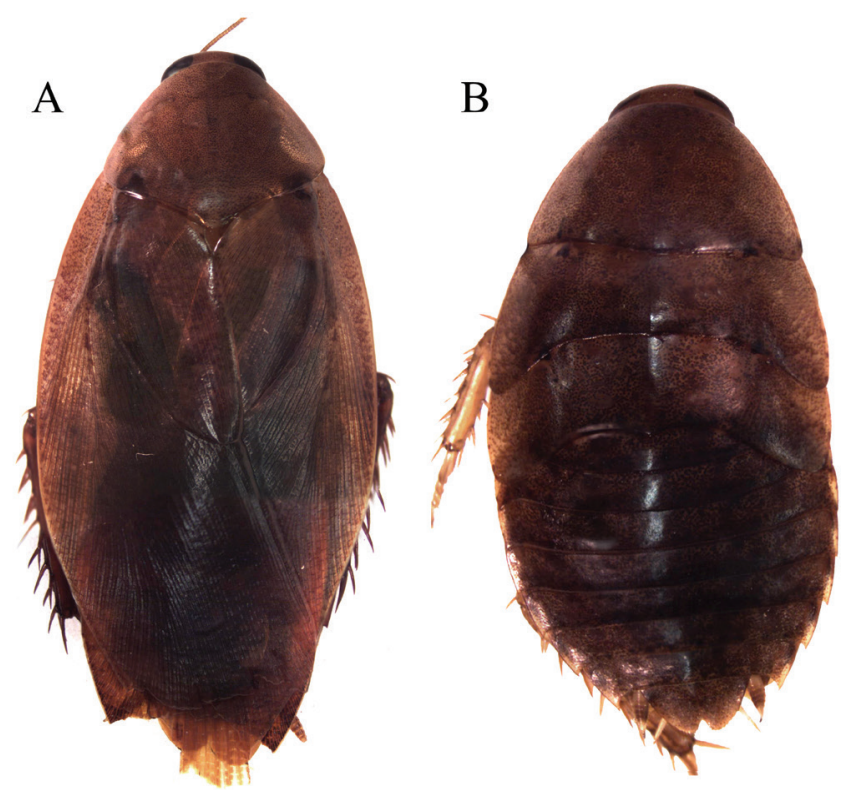

Figure 4. Epilampra sodali. A Adult male dorsal view (DECBA0401) B Juvenile dorsal view (DECBA2163). Add scale bar.

Collection locale. CEIBA Biological Station, Madewini, Guyana.

GPS: $6^{\circ} 29^{\prime} \mathrm{N}, 58^{\circ} 13^{\prime} \mathrm{W}$.

Date: 31 - July to 6 - August - 2011.

Collectors. Dominic A. Evangelista, Ian Biazzo, Manpreet K. Kohli, Melissa Sanchez-Herrera, Nicole Sroczinski, and Jessica L. Ware.

Collection/ecological information. This specimen was collected at a light trap.

Morphological identification. This specimen agrees with the description the synonym E. cinnamomea (Hebard 1926).

\section{Juvenile}

Voucher number: DECBA1702.

GenBank accession number: KF155068.

Collection locale. CEIBA Biological Station, Madewini, Guyana.

GPS: $6^{\circ} 29^{\prime} \mathrm{N}, 58^{\circ} 13^{\prime} \mathrm{W}$.

Date: 27 - December - 2011.

Collectors. Dominic A. Evangelista, Ian Biazzo, Joseph A. Evangelista, Paul Frandsen, William R. Kuhn and Jessica L. Ware.

\section{Juvenile}

Voucher number: DECBA1701.

GenBank accession number: KF155069.

Collection locale. CEIBA Biological Station, Madewini, Guyana. 
GPS: $6^{\circ} 29^{\prime} \mathrm{N}, 58^{\circ} 13^{\prime} \mathrm{W}$.

Date: 10 - January - 2012.

Collectors. Dominic A. Evangelista, Ian Biazzo, Joseph A. Evangelista, Paul Frandsen, William R. Kuhn and Jessica L. Ware.

Collection/ecological information. Both of these juvenile specimens were collected at the edge of a small pond.

Genetic information and evolutionary placement. These three specimens (previous reported as "Blaberidae sp. 04") were placed in the same clade with 90\% bootstrap support.

Known geographic distribution. Venezuela, Guyana (new record), Suriname, French Guiana and Brazil

\section{Subfamily: Zetoborinae}

\section{Thanatophyllum akinetum Grandcolas, 1991}

Materials. Adult §̋ Figure 5.

Voucher number: DECBA0611.

GenBank accession number: KF155066.

Collection locale. CEIBA Biological Station, Madewini, Guyana.

GPS: 6²9'N, 58²'W.

Date: 28 - December - 2011.

Collectors. Dominic A. Evangelista, Ian Biazzo, Joseph A. Evangelista, Paul Frandsen, William R. Kuhn and Jessica L. Ware.

Collection/ecological information. This specimen was collected by hand on vegetation in an uplands secondary forest.

Morphological identification. This specimen agrees with the description of the head and male genitalia of Grandcolas (1990).

Known geographic distribution. Guyana (new record) and French Guiana.

\section{Family: "Ectobiidae"}

Subfamily: Anaplectinae

Anaplecta parviceps (Walker, 1868)

Materials. Adult ふ̋ Figure 6.

Voucher number: DECBA1843.

GenBank accession number: KF155137.

Collection locale. CEIBA Biological Station, Madewini, Guyana.

GPS: 6²9'N, 58² $13^{\prime} \mathrm{W}$.

Date: 16 -August - 2012 . 

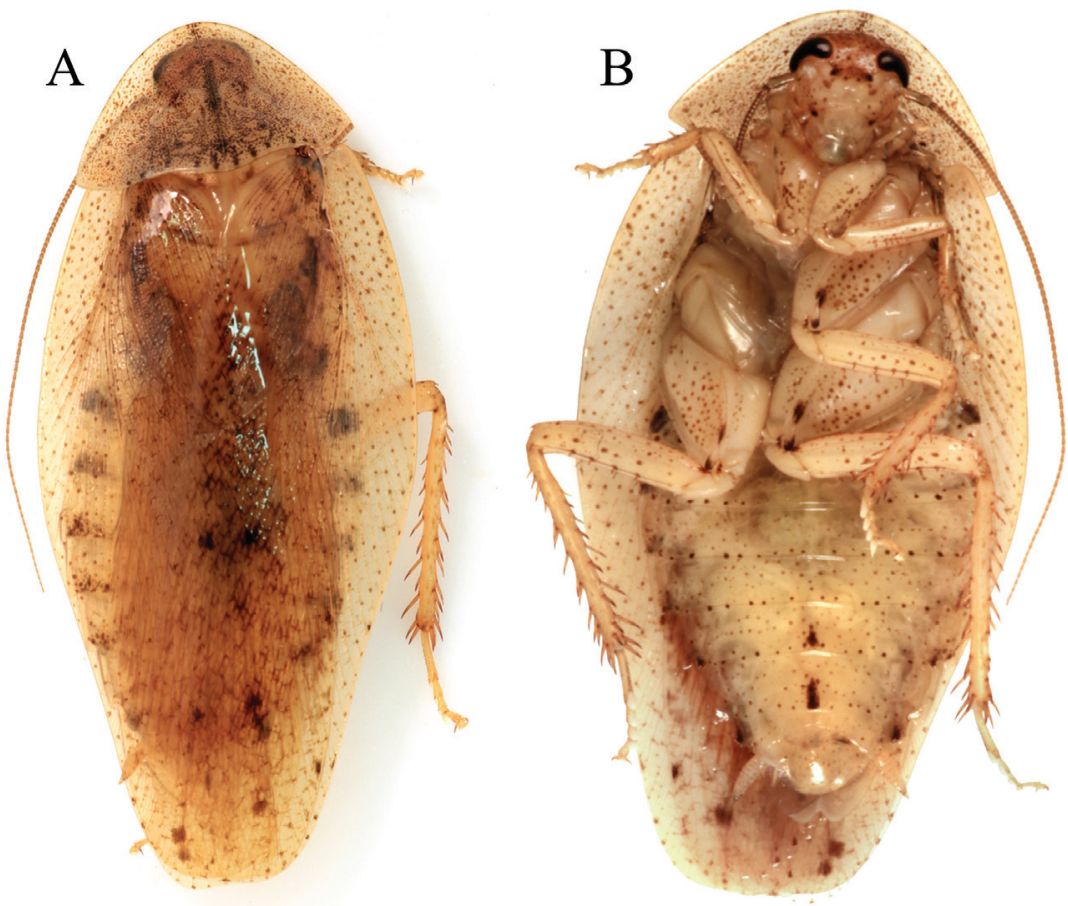

Figure 5. Thanatophyllum akinetum adult male (DECBA0611). A Dorsal view B Ventral view.

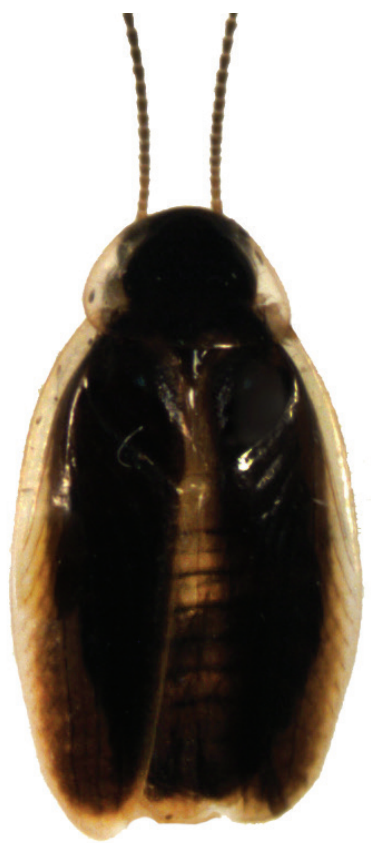

Figure 6. Anaplecta parviceps adult male (DECBA1843). 
Collectors. Dominic A. Evangelista and William R. Kuhn.

Collection/ecological information. This specimen and another adult male (Voucher number: DECBA1841) were collected at a light trap near the camp of CEIBA Biological Station on the date noted above. A juvenile of this species was also collected at the same locale, found crawling through a benab between 21 and 24 of August 2012 (Voucher number: DECBA1842).

Morphological identification. The specimen agrees with the description of the synonym A. insignis of Hebard (1926). Other specimens were identified by comparison with specimen DECBA1843.

Genetic information and evolutionary placement. The COI barcodes of this specimen (previously reported as "Blattodea sp. 18") falls sister to another specimen identified as Anaplecta sp. (previously reported as "Ectobiidae sp. 04"; Voucher number: DEDSM0111; GenBank accession number: KF155041) but with 25\% bootstrap support. This other species is not reported in this paper due to an uncertainty in specific identification.

Known geographic distribution. Guyana, Suriname, French Guiana, Brazil (Rio de Janeiro), Brazil (Pará), and Brazil (Amapá).

\section{Subfamily: Blattellinae}

\section{Anisopygia decora Hebard, 1926}

Materials. Adult $q$ Figure 7.

Voucher number: DEKBO0504.

Collection locale. Capuchin Trail, Karanambo Ranch, Rupununi, Guyana.

GPS: $3^{\circ} 44^{\prime} 43.70 " \mathrm{~N}, 59^{\circ} 18^{\prime} 51.88^{\prime \prime W}$.

Date: 10 - June - 2013.

Collectors. Dominic A. Evangelista, Oswin Ambrose, Susan George, and Megan M. Wilson.

Collection/ecological information. This specimen was collected by hand in an undisturbed forested area. This is the first record of this specimen from Guyana.

Morphological identification. This specimen was identified by comparison with Hebard's description (1926).

Known geographic distribution. Guyana (new record) and French Guiana.

\section{Ischnoptera atrata Hebard, 1916}

Materials. Adult $\widehat{O}$.

Voucher number: DECBA2153.

Collection locale. CEIBA Biological Station, Madewini, Guyana.

GPS: $6^{\circ} 29^{\prime} \mathrm{N}, 58^{\circ} 13^{\prime} \mathrm{W}$. 


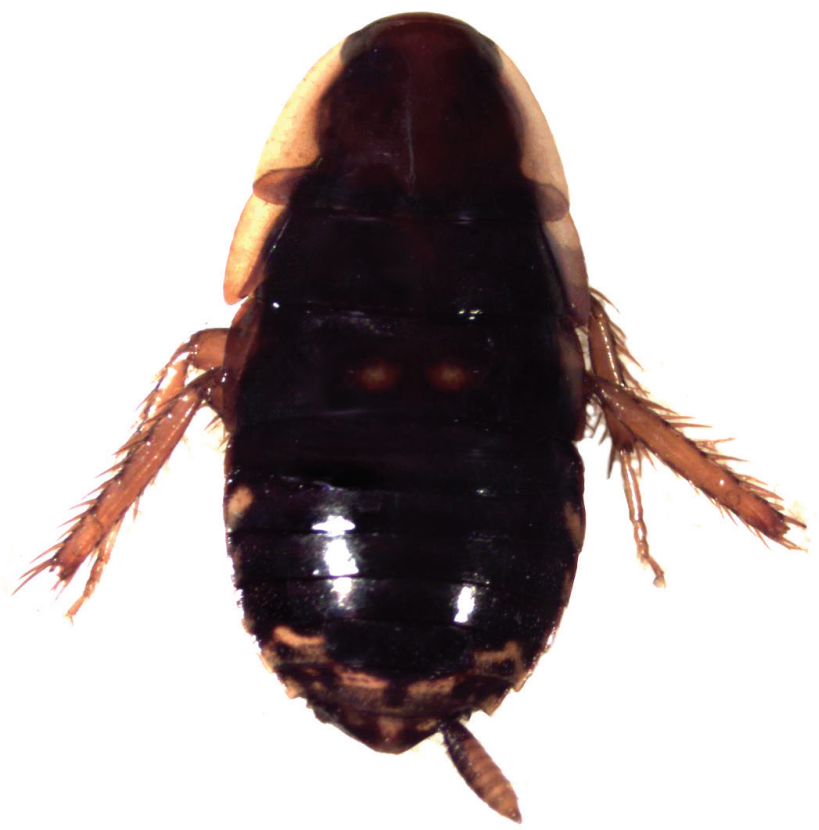

Figure 7. Anisopygia decora adult female (DEKBO0504).

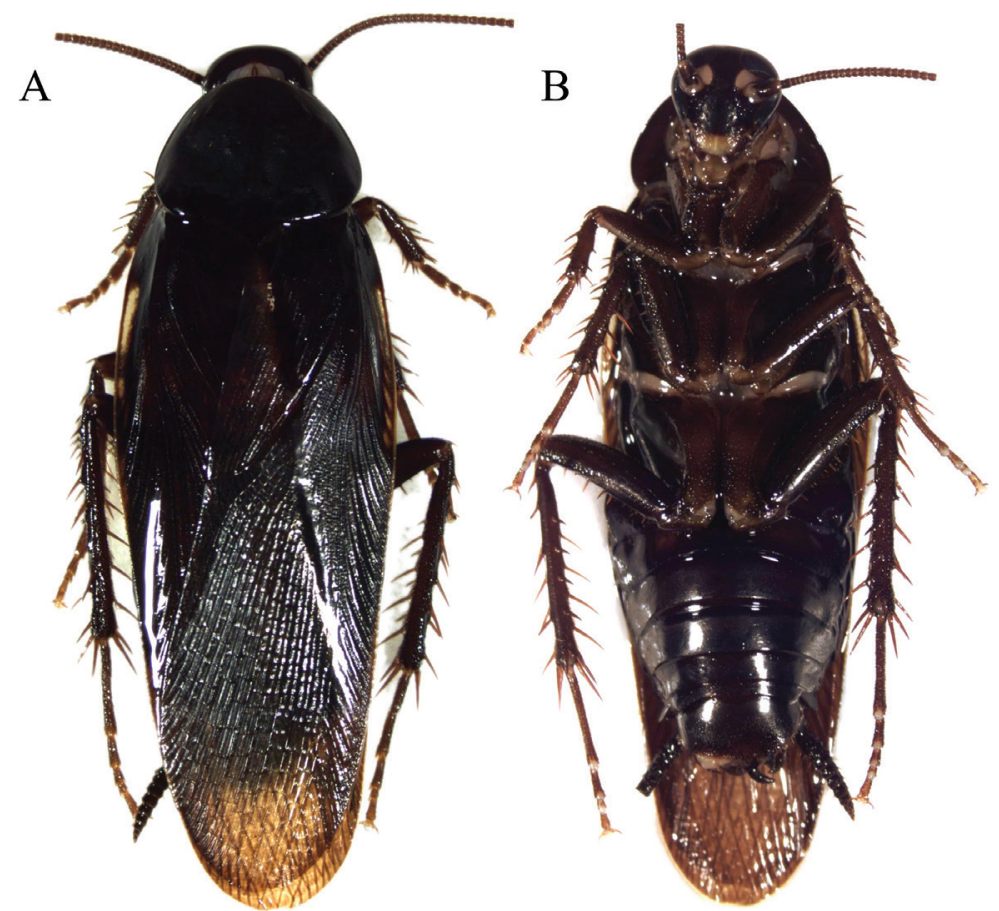

Figure 8. Ischnoptera atrata adult male (DEKBO0594). A Dorsal view B Ventral view. 
Collection date: December - 2011.

Collectors. Dominic Evangelista, Ian Biazzo, Joseph A. Evangelista, Paul Frandsen, William R. Kuhn, and Jessica L. Ware.

Collection/ecological information. This specimen was collected in a pitfall trap baited with beer in an uplands secondary forest area.

Adult đ̋ Figure 8.

Voucher number: DEKBO0594.

Collection locale. Karanamabu Ranch, Rupununi, Guyana.

GPS: 345'0.1"N, 59¹8'53.7"W.

Collection date: 10 - June - 2013.

Collectors. Dominic A. Evangelista, Oswin Ambrose, Susan George, and Megan M. Wilson.

Collection/ecological information. This specimen was collected in a pitfall trap baited with beer in a forest proximal to the Rupununi River.

Morphological identification. Both specimens mostly agree with the description and figures of Hebard (1916). However, there are slight differences in the supra-anal plate when compared to Hebard's illustration. The white region on the SA plate of our specimen is slightly larger than in Hebard's illustration. It is possible that this is a different species than that described by Hebard, but this cannot be fully determined without a full phylogenetic treatment of sexual morphology and genetic information of individuals from both Trinidad and Guyana.

Known geographic distribution. Trinidad and Tobago, and Guyana

Xestoblatta berenbaumae Evangelista, Kaplan, \& Ware, sp. n. http://zoobank.org/0DCFF043-F783-49E4-8576-4A2AD402AF82

Authors of description. Evangelista, Kaplan, \& Ware.

Holotype. Adult $\widehat{\jmath}$ Figure 9B-E, G.

Voucher number: DECBA2109.

Type locality. CEIBA Biological Station, Madewini, Guyana.

GPS: 6²9'N, 58²'W.

Collection date: 17 to 18 - August - 2012 .

Collectors. Dominic A. Evangelista and William R. Kuhn.

Type information. The holotype specimen is stored in ethanol with genitalia in a separate ethanol vial and is deposited at the Center for Biodiversity at the University of Guyana.

Collection/ecological information. This specimen was collected in a pitfall trap baited with beer and fruit in an uplands secondary forest in CEIBA Biological Station.

Morphological identification. This specimen was identified as Xestoblatta Hebard, 1916 by the position of the hooked phallomere (left), the presence of the external modification of the tergum as part of the dorsal tergal gland (Figure 9A), incomplete 


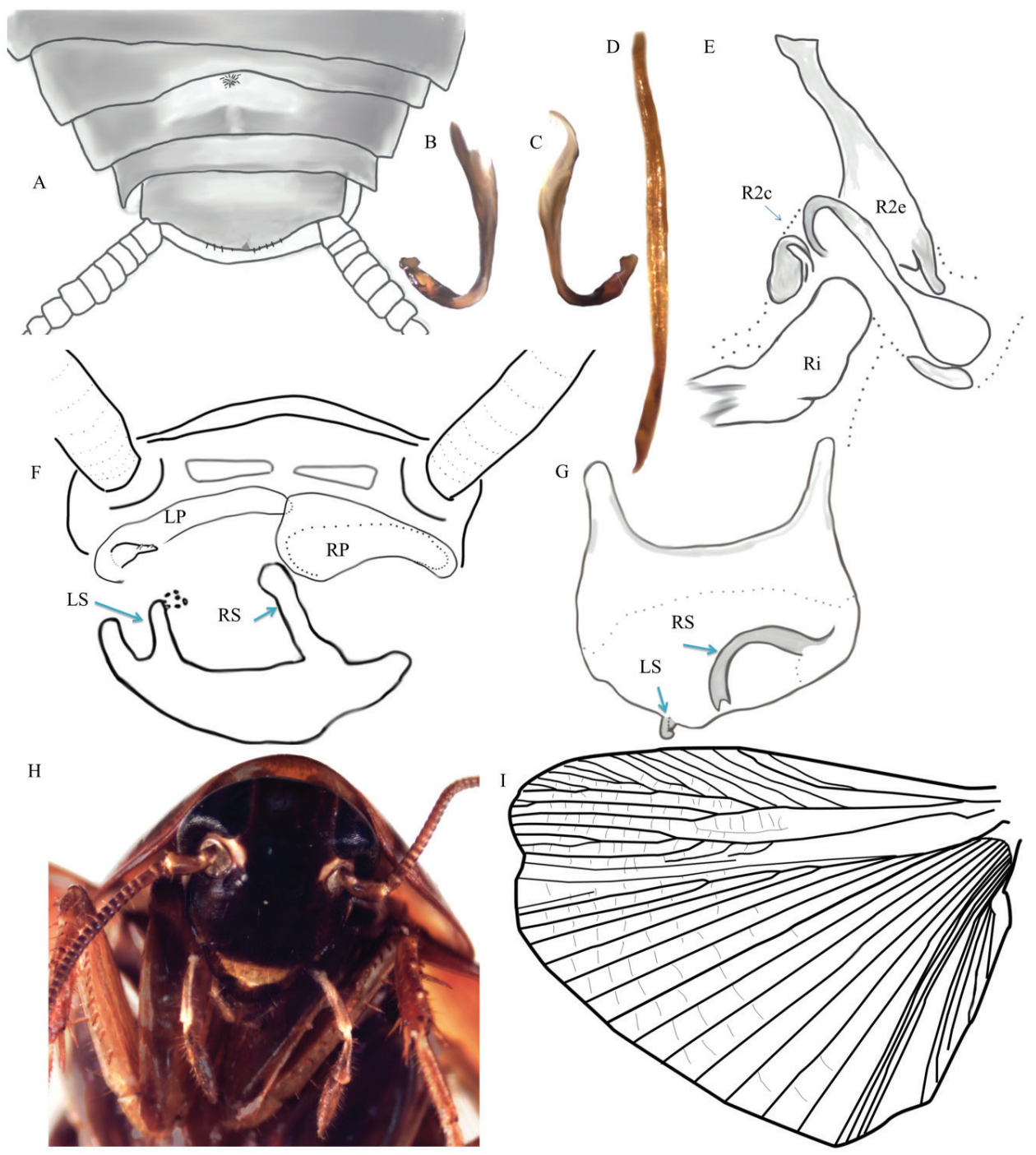

Figure 9. Xestoblatta berenbaumae sp. n. A Dorsal view of abdomen showing the simple tergal gland (DECBA2023) B, C Hooked left phallomere D Ventral medial phallomere (L2vm) E Right phallomere. R2e - external sclerite, R2i - internal sclerite, R2c - cleft sclerite F Posterior view of abdomen showing paraprocts and subgenital plate. RS-right stylus, LS-left stylus with small translucent ball at tip, LP-left paraproct reduced and specialized with polydentate spine, RP-unspecialized right paraproct. Illustration is a composite of multiple individuals $\mathbf{G}$ Dorsal view of sub-genital plate (DECBA1967) $\mathbf{H}$ Head of adult male I Hindwing (DECBA0801). Photos and illustrations contributed by Kayla Kaplan and Dominic A. Evangelista.

rami on the ulnar vein of the hind wing (Figure 9I) and the spination (type A) on the ventro-anterior margin of the fore-femur.

Holotype morphological description. Head uniformly colored a deep mahogany. Clypeus pale buffy. Ocellar spots easily distinguishable, smaller than antennal 
pits and white. Head otherwise without distinguishing features. Ocellar spots slightly closer together than eyes. Facial grooves on lateral most edge. See Figure 9H for a representative photo of the head.

Pronotum a uniformly reddish mahogany color (Figure 10A). Medial expansion on posterior margin of pronotum is barely noticeable. Ventral margin of pronotum not lined with hairs. Anterior margin of pronotum significantly conformed around the head. Leg coloration deep orange amber. Coxae with some diffuse black regions. Ventro-anterior margin of fore-femur with 14 (left) or 13 (right) spines decreasing in size from basal to apical, one slightly larger pre-apical spine and one large apical spine (16 total left, 15 total right). Ventro-posterior margin of forelimbs with 4 large spines and 1 apical spine. Ventro-anterior margin of middle leg has seven large spines and one apical spine. Middle leg also with one large genicular spine. Hind leg ventro-anterior margin has six spines, one apical spine, and one genicular spine. Pulvilli present on all tarsomeres. Arolia present but not surpassing the tips of the pretarsal claws. Claws symmetrical and unspecialized.

Ulnar vein with three incomplete rami and three complete rami (Figure 9I). Tegmina reddish mahogany with small patch of white under the base of the subcostal vein.

Supra-anal plate subtriangular with a blunt tip from dorsal view. Left paraproct modified into a tri-dentate spine (Figure 9F; bi-dentate in some other specimens). Sub-genital plate has both styli highly modified (Figure 9F, G). The right stylus is projecting dorso-medially from posterior margin, curving back posteriorly and terminating in a shape reminiscent of a bifurcated serpentine tongue. Left stylus projecting dorsally, shorter than right stylus and tipped with a small, translucent, irregularly shaped ball (Figure 9F, G).

Left phallomere (Figure 9B, C) hooked in apical third. (Hooked phallomere is about $1.5 \mathrm{~mm}$ long). Medial phallomere (Figure 9D) approximately three times the length of the left phallomere, roughly uniform width, and a slight slender curve in the posterior end. R2c (Figure 9E) divided into two sclerites that form dual concave cups that meet dorsally.

Dorsal modification of terga as part of the dorsal tergal gland. Modification represented by a small patch of hairs with a concave semi-circular modification of the margin of the segment anterior to the gland. See Figure 9A for an illustration of a representative dorsum.

Medium sized hairs $(-2 \mathrm{~mm})$ covering entire body roughly uniformly, yet sparsely.

Other adult male paratypes. Voucher numbers: DECBA1967, DECBA0801, DECBA1958, DECBA2182, DECBA2092, DECBA2039

Collection/ecological information. All additional male individuals reported here were collected in leaf litter pitfall traps baited with beer at various locations (dryer secondary uplands forest and wet primary lowlands forest) in CEIBA biological station.

Adult female paratype morphological description.Voucher number: DECBA2074.

Head slightly darker in color than male with a more reflective surface. Other features of head similar to male. 

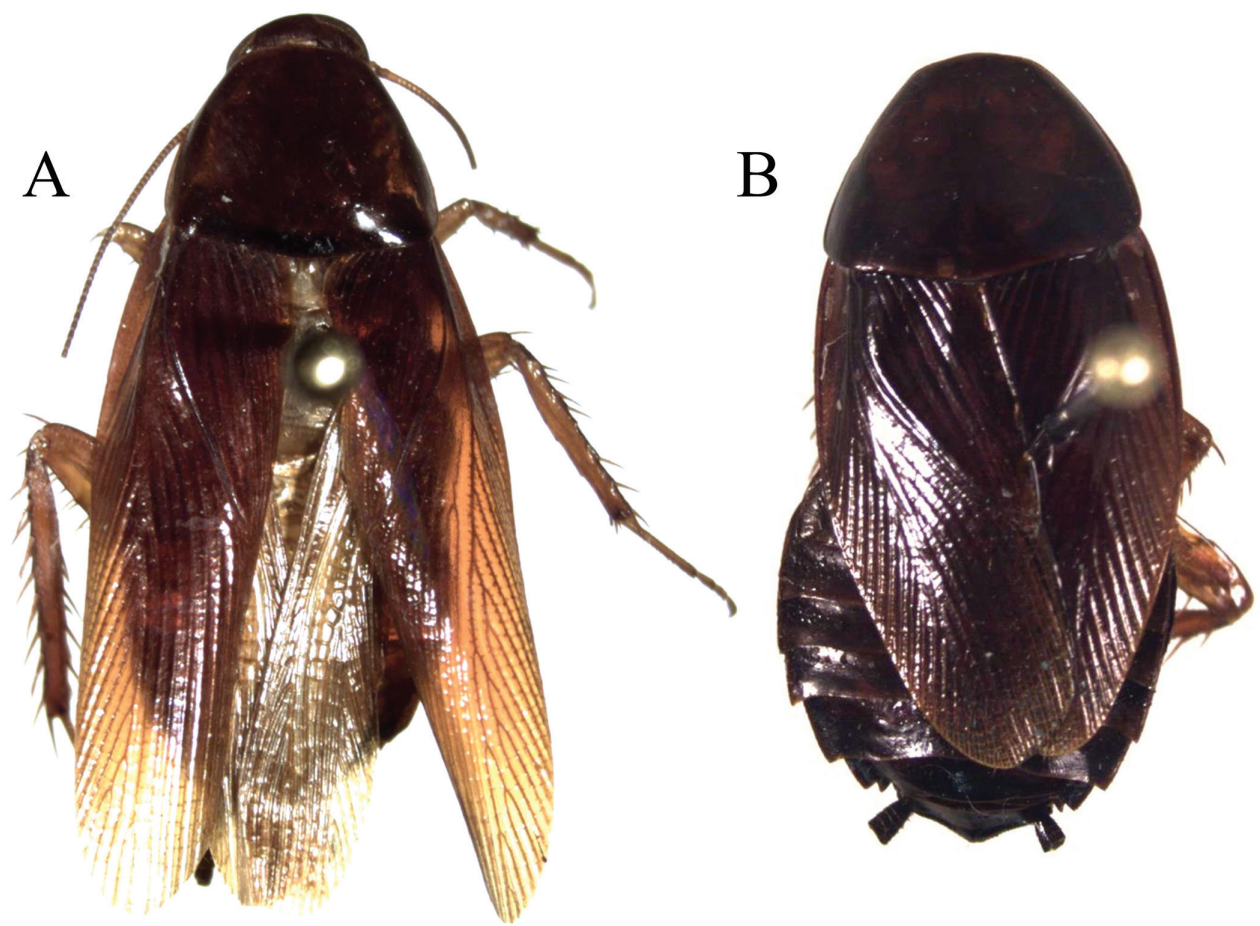

Figure 10. Xestoblatta berenbaumae sp. n. A Adult male dorsal view (DECBA2182) B Adult female dorsal view (DECBA2210).

Description of legs similar or identical to that of male with the following spination on the ventro-anterior margin of fore-femur: 13 (left) and 12 (right) spines decreasing in size from basal to apical, two larger preapical spines and one large apical spine (16 total left and 15 total right). Ventro-posterior margin of fore-femur four large spines and one apical spine. Ventro-anterior margin of mid-leg with seven large spines, one apical spine, and one genicular spine. Ventro-anterior margin of hind-leg with five large spines, one apical spine, and one genicular spine.

Tegmina and wings reduced and not reaching end of abdomen. Three incomplete and three complete rami on ulnar vein. Ulnar vein very faint in the reduced wings of the female (Figure 10B; Table 3).

Pronotum matches description of the male.

Subgenital plate slightly more abbreviated than in male. Paraprocts simple and unspecialized. Sub-genital plate simple and symmetrical.

Other adult female paratypes. Voucher numbers: DECBA1787, DECBA1791, DECBA1792, and DECBA1793

Collection/ecological information. All additional female individuals reported here were collected in leaf litter pitfall traps baited with beer in an uplands secondary forest at CEIBA biological station. 


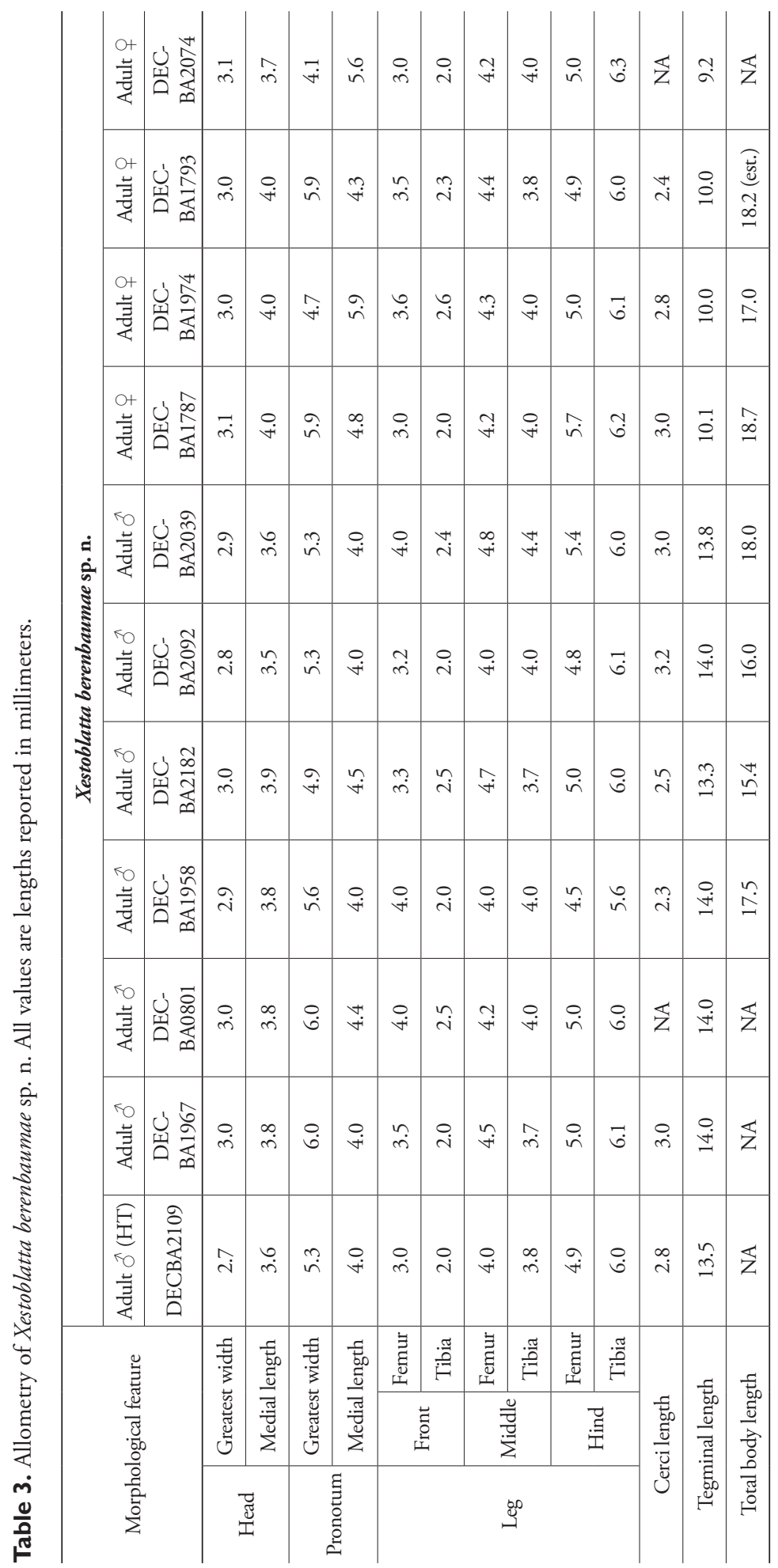


Summary of female morphology. All individuals match the description of the above female and have the following spination on the vento-anterior margin of the fore-limb: 13 spines decreasing in size from basal to apical, one or two slightly larger preapical spines and one large apical spine making a total of 15 or 16 spines.

Juvenile paratypes. Voucher numbers: DECBA1788, DECBA1789, DECBA1790, DECBA1796.

Collection/ecological information. All additional juvenile individuals reported here were collected in leaf litter pitfall traps baited with beer in an uplands secondary forest at CEIBA biological station.

Summary of juvenile morphology. Juveniles are apterous and largely match the morphology of adults except for in the following. Simple styli present on the subgenital plate in some individuals but are short and abbreviated. Spines on ventro-anterior margin of forelimb are as follows: 12 to 14 spines decreasing in size basally to apically, one or two slightly larger preapical spines and one large apical spine making a sum total of 15 or 16 total spines.

Molecular data and evolutionary placement. Vouchers numbers and GenBank accession numbers: DECBA1791 - KF155114, DECBA1789 - KF155105, DECBA0801 - CBA0801, DECBA1827 - KF155103, DECBA1826 - KF155107, DECBA1814 KF155115. The clade containing the above haplotypes (formerly reported as "Blattodea sp. 1 ") is supported by $96 \%$ bootstrap support and the haplotypes are nearly identical.

Diagnostic features of $X$. berenbaumae. The morphology of modified styles on the subgenital plate is the most useful trait for discerning this species with other Xestoblatta Hebard, 1916. The simple dorsal tergal gland, shape of the paraprocts (left modified into a tri-dentate or bi-dentate spine), and morphology of the internal genital sclerites of the male are also useful in identifying this species. Unfortunately the adult females and juveniles are largely lacking obvious identifying characteristics and there may be errors made in associating juveniles to the adults without the use of genetic information.

Etymology. We give this species the specific epithet "berenbaumae" in honor of the esteemed entomologist, Dr. May Berenbaum, who has made huge contributions to entomology through scientific products, service and public outreach.

Known geographic distribution. Guyana

\section{Xestoblatta agautierae Grandcolas, 1992}

Materials. Adult ô.

Voucher number: DEKBO0827.

Collection locale. Wilson's pond trail (Honey pond trail), Karanambu Ranch, Rupununi, Guyana.

GPS: $3^{\circ} 44^{\prime} 42.36 " \mathrm{~N}, 59^{\circ} 19^{\prime} 15.21^{\prime \prime W}$.

Collection date: 10 - June - 2013.

Collectors. Dominic A. Evangelista, Oswin Ambrose, Susan George, and Megan M. Wilson. 


\section{Adult ㅇ․}

Voucher number: DEKBO0826.

Collection locale. Forest Island "Darwin", Karanambu Ranch, Rupununi, Guyana. GPS: $3^{\circ} 47^{\prime} 47.62 " \mathrm{~N}, 59^{\circ} 22^{\prime} 6.77^{\prime \prime W}$.

Collection date: 14 - June - 2013.

Collectors. Dominic A. Evangelista, Oswin Ambrose, Susan George, and Megan M. Wilson.

Collection/ecological information. Both specimens above were collected in pitfall traps baited with beer in the forests of the Rupununi savannah.

Morphological identification. The left genital phallomere, right genital phallomere, absence of a dorsal tergal gland and body coloration match closely with the species description (Grandcolas 1992a). The styli differ slightly to the illustrations in the original description in that the left stylus of our specimen is shorter and originates more medially. The female was associated to the male by comparison of gross morphology and body coloration. See Figure 11 for photos of adult male and adult female.

Collection/ecological information for other specimens not reported here. We collected many individuals of this species from most forested areas surrounding Karanambu Ranch. We collected only one individual of this species in a similar trap at the edge of a forest, near open savannah. We found this species and $X$. berenbaumae sp. n. to be extremely abundant in their respective localities ( $>100$ individuals of each collected). However, both are previously unreported for Guyana. We believe this can be attributed to the fact that we used beer and fermenting fruit to bait out pitfall traps. As Gurney (1939) reports, Xestoblatta Hebard, 1916 were rare in collections until the contributions of an entomologist trapping fruit flies in Panama. We can speculate that these fruit flies were also collected with some sort of aromatic bait (as this is common for fruit fly trapping) that attracted the Xestoblatta Hebard, 1916 as by-catch.

Known geographic distribution. Guyana (new record) and French Guiana.

\section{Subfamily: Nyctiborinae}

\section{Nyctibora dichropoda Hebard, 1926}

\section{Materials. Adult $\widehat{O}$ Figure 12.}

Voucher number: DECBA0302.

GenBank accession number: KF155061.

Collection locale. CEIBA Biological Station, Madewini, Guyana.

GPS: $6^{\circ} 29^{\prime} \mathrm{N}, 58^{\circ} 13^{\prime} \mathrm{W}$.

Collection date: 29 - July - 2011.

Collectors. Dominic A. Evangelista, Ian Biazzo, Manpreet K. Kohli, Melissa Sanchez-Herrera, Nicole Sroczinski and Jessica L. Ware.

Collection/ecological information. This specimen was collected in the leaf litter. 

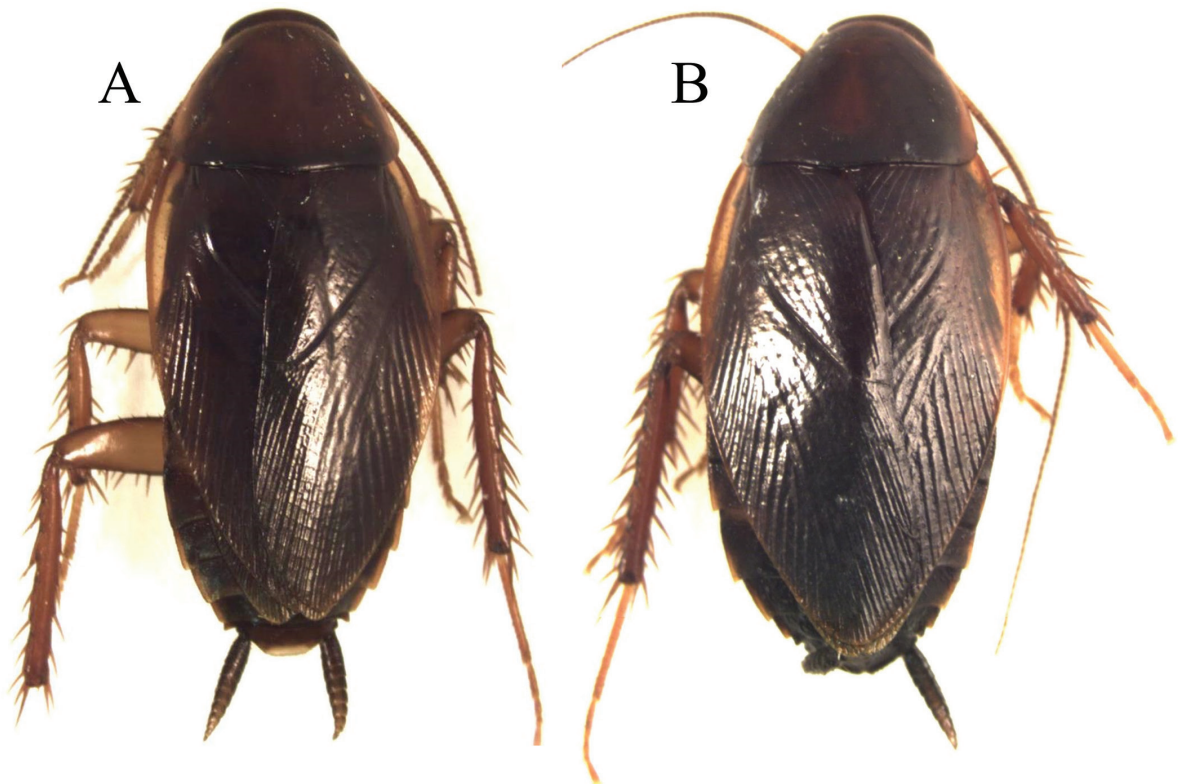

Figure I I. Xestoblatta agautierae. A Adult male dorsal view (DEKBO0442) B Adult female dorsal view (DEKBO0445).
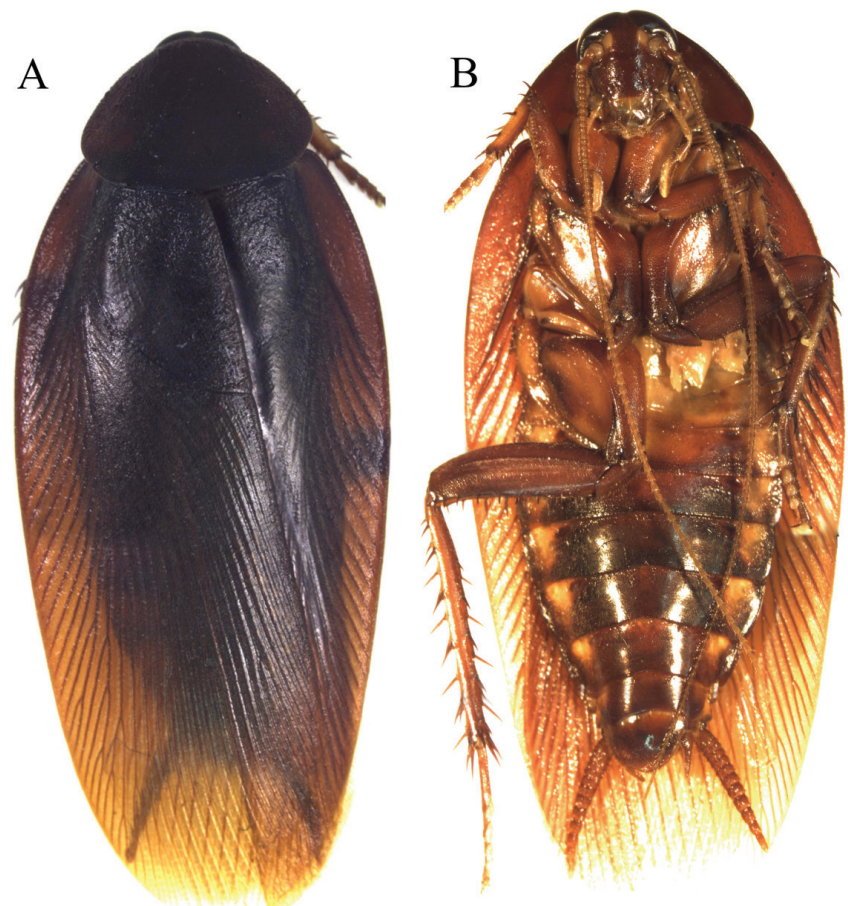

Figure I2. Nyctibora dichropoda adult male (DECBA0302). A Dorsal view B Ventral view. 
Morphological identification. This specimen matches the illustration and description by Hebard (1926) in the "striking pale" coloration on the surfaces of the tibiae, the definitive character for this species. However, the male we have is much larger than that which he described. It is matching in all other ways.

Molecular identification. The COI barcodes of this specimen are close to an adult female (Voucher number: DECBA0235; GenBank accession number: KF155062) and juvenile specimen (Voucher number: DECBA0104; GenBank accession number: KF155024) of Nyctibora. Based on both genetic distance and morphological dissimilarity, these individuals are likely members of a separate species. We do not report them further here.

Known geographic distribution. Guyana (new record), Suriname and French Guiana.

\section{Subfamily: Pseudophyllodromiinae}

\section{Chorisoneura inversa Hebard, 1926}

Materials. Adult ô Figure 13.

Voucher number: DECBA1782.

GenBank accession number: KF155130.

Collection locale. CEIBA Biological Station, Madewini, Guyana.

GPS: $6^{\circ} 29^{\prime} \mathrm{N}, 58^{\circ} 13^{\prime} \mathrm{W}$.

Date: 7 to 11 - August -2013 .

Collectors. Dominic A. Evangelista, Ian Biazzo, Manpreet K. Kohli, Melissa Sanchez-Herrera, Nicole Sroczinski and, Jessica L. Ware.

Morphological identification. This individual was recognizable when comparing to the description of Hebard (1926) and the presence of the anteriorly pointing "V" shape on pronotum.

Genetic information and evolutionary placement. As discussed below, this specimen was placed near Calhypnorna Saussure \& Zehntner, 1893 with 75\% bootstrap support.

Known geographic distribution. Guyana, Suriname, French Guiana and Brazil.

\section{Dendroblatta callizona Rehn, 1928}

Materials. Adult $\bigcirc$ Figure 14.

Voucher number: DECBA0805.

Collection locale. CEIBA Biological Station, Madewini, Guyana.

GPS: $6^{\circ} 29^{\prime} 57.75^{\prime \prime N}, 58^{\circ} 13^{\prime} 7.28^{\prime \prime W}$.

Date: 14 - August -2011 .

Collectors. Dominic A. Evangelista, Ian Biazzo, Manpreet K. Kohli, Melissa Sanchez-Herrera, Nicole Sroczinski, and Jessica L. Ware. 


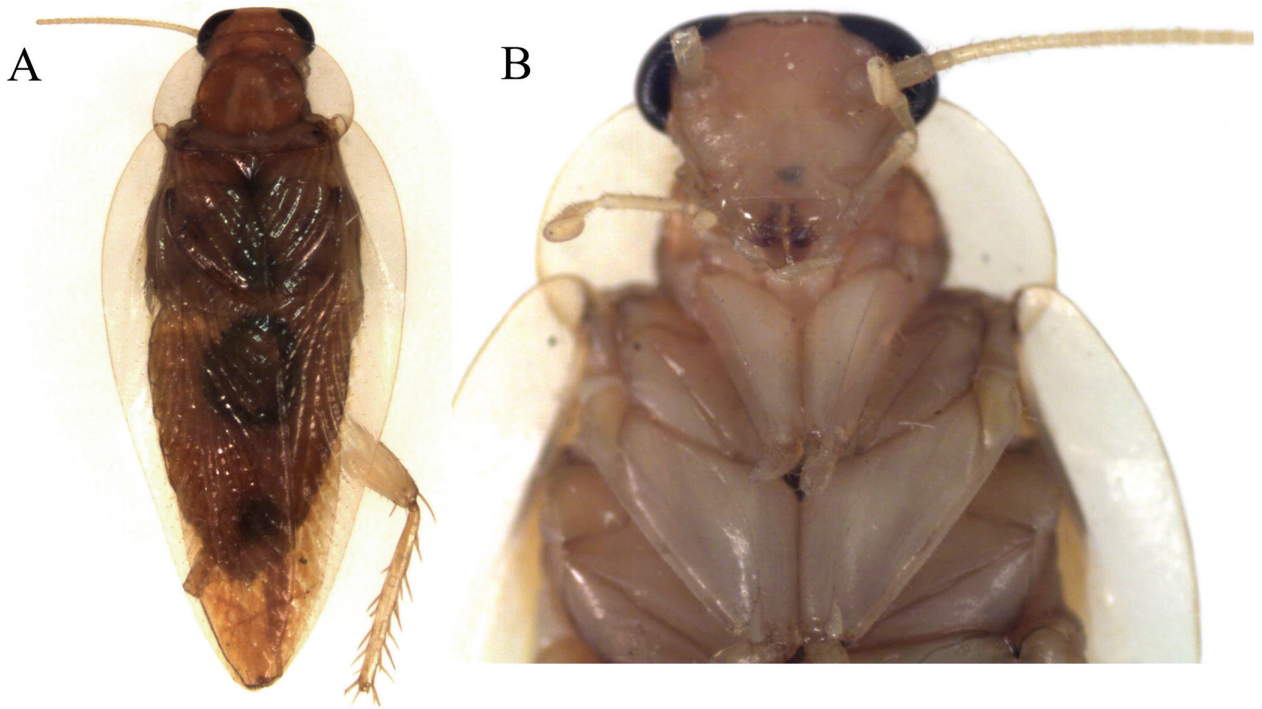

Figure 13. Chorisoneura inversa adult male (DECBA1782). A Dorsal view B Ventral view of head.

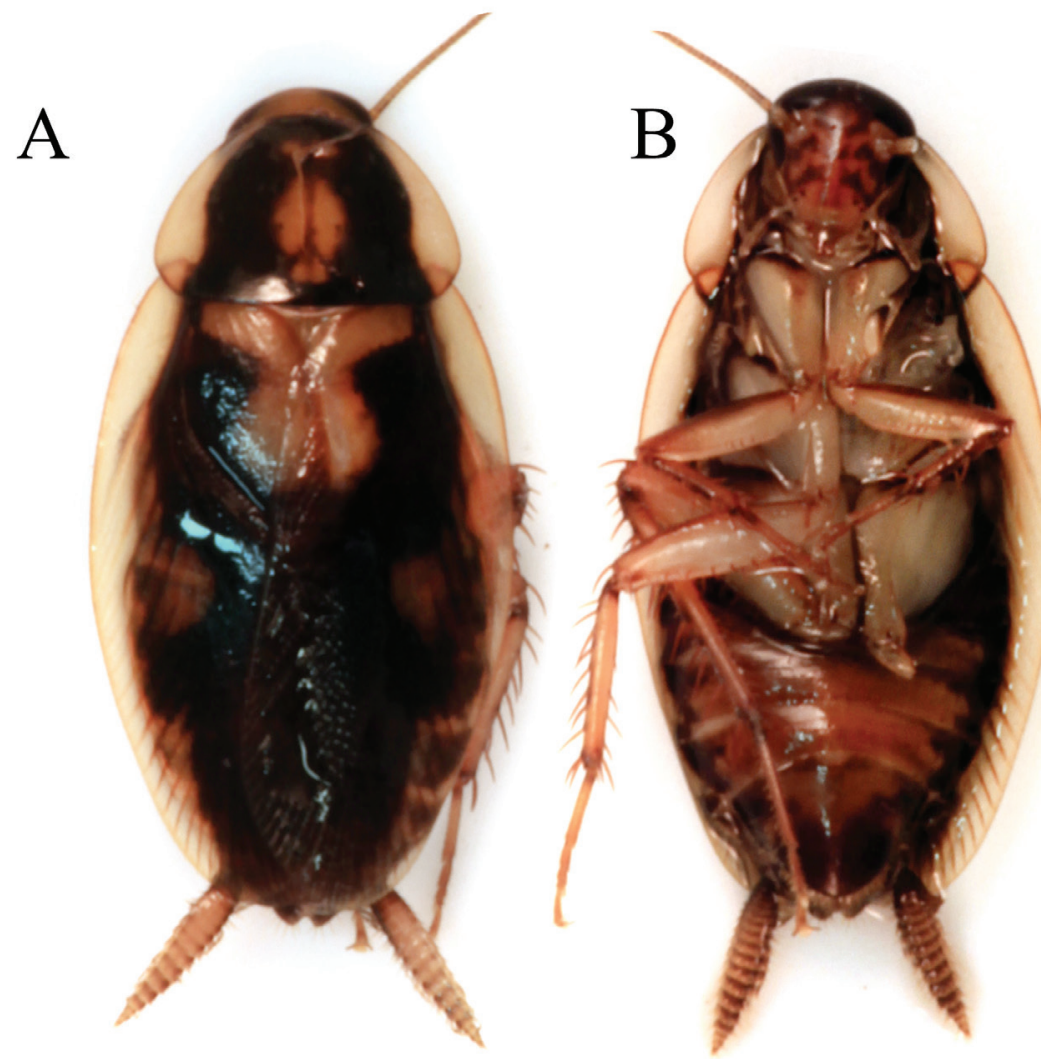

Figure 14. Dendroblatta callizona adult female (DECBA0805). A Dorsal view B Ventral view. 
Juvenile

Voucher number. DECBA0901.

GenBank accession number: KF155067.

Collection locale. CEIBA Biological Station, Madewini, Guyana.

GPS: 62 $29^{\prime} 57.75^{\prime \prime} \mathrm{N}, 58^{\circ} 13^{\prime} 7.28^{\prime \prime W}$.

Date: 13 - August - 2011.

Collectors. Dominic A. Evangelista, Ian Biazzo, Manpreet K. Kohli, Melissa Sanchez-Herrera, Nicole Sroczinski, and Jessica L. Ware.

Collection/ecological information. Both of these specimens were collected in a cup baited with beer placed in the canopy. The cup was tied to the trunk of a tree 13.8 meters above the ground. The tree chosen was close to a swampy primary forest area and on the edge of grassy hillside (most likely a plot that had been burned in the past). There were traps placed in the same tree at other heights but both individuals of this species were caught in this particular trap.

Morphological identification. Our female specimen of D. callizona Rehn, 1928 is within the variation described by Rehn (1928). The juvenile specimen was identified by comparison with the adult and using genetic data as well.

Genetic information. In the tree of Evangelista et al. (2014) this species is placed near two individuals reported as "Ectobiidae sp. 10". The morphology of these specimens is consistent with Dendroblatta cnephaia Hebard, 1926, although we do not report them here because of a lack of adults to confirm identification.

Known geographic distribution. Trinidad and Tobago, Guyana, and Suriname.

\section{Calhypnorna sp. A}

Authors of the description. Evangelista, Wilson, \& Ware.

Materials. Juvenile Figure 15.

Voucher number: DECBA1802.

GenBank accession number: KF155118.

Collection locale. CEIBA Biological Station, Madewini, Guyana.

GPS: $6^{\circ} 29^{\prime} \mathrm{N}, 58^{\circ} 13^{\prime} \mathrm{W}$.

Collection date: 15 - August -2012 .

Collectors. Dominic A. Evangelista and William R. Kuhn.

Specimen information. This specimen is stored in ethanol and is deposited in the Center for Biodiversity at the University of Guyana.

Identification and differential diagnosis. We identified this specimen as Calhypnorna based on the following comparisons. Our specimen is not lacking an interocular carina as in Hypnornoides (Rehn 1917). Our specimen also has a definitively truncate posterior margin of the pronotum (Figure 15B), which differentiates it from Euhypnorna (Hebard 1921). Our specimen is lacking the hairs covering most of the body as in Hypnorna (1893) and most closely matches the illustration of Calhypnorna by Saussure and Zehntner (1893). 


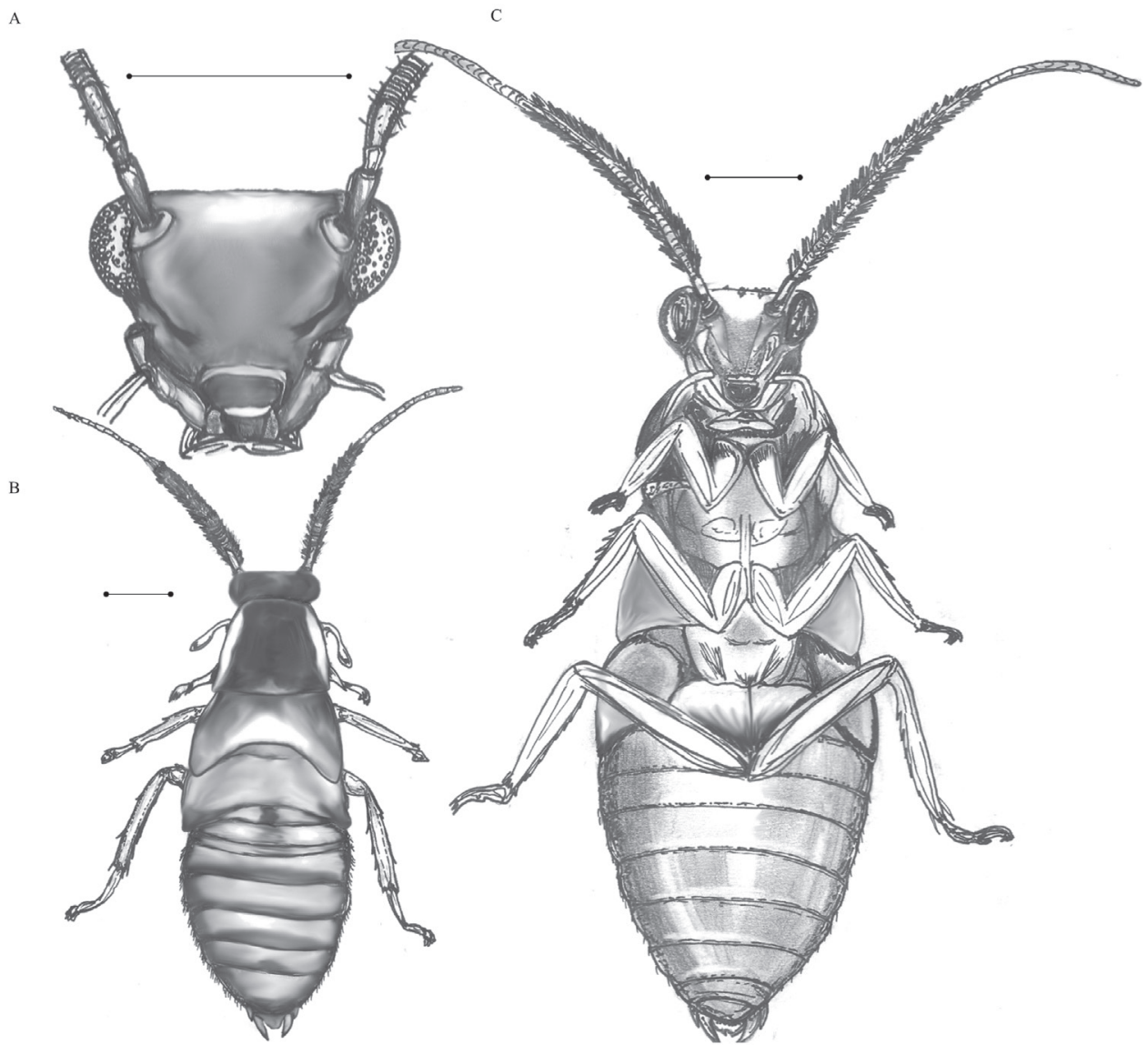

Figure I 5. Calhypnorna sp. A Ventral view of head B Dorsal view of body C Ventral view of body. Scale bar $=1 \mathrm{~mm}$. Illustrations contributed by Megan M. Wilson.

Description. The specimen is a juvenile that is likely in its penultimate instar. Overall, the body shape is elongated for a typical cockroach, and even for a typical Pseudophyllodromiinae. A large portion of the head is visible from a dorsal perspective, and reaches anteriorly past the pronotum significantly. The black coloration on the pronotum is the same width as the width of the head where it meets with the pronotal margin (Figure 15B).

Antennae are hirsute to nearly plumose. The antennae are slightly clubbed basally with the widest point occurring at first segment of the flagellum. There are two major color regions of the antennae: a dark basal region and a light distal region. The dark basal region begins as slightly lighter than the remainder but becomes a dark black color by the end of the dark region. The $25^{\text {th }}$ segment of the antennae is the final dark segment. The $26^{\text {th }}$ antennal segment begins the light region of the antennae. The $26^{\text {th }}$ or $27^{\text {th }}$ and subsequent segments are nearly white, becoming more brownish orange 
after the $7^{\text {th }}$ white segment $(33$ total). The total number of antennal segments on the specimen is 38 (left) and 44 (right).

The head is very large in relation to the remainder of the body, triangular, and wider than typical for a Pseudophyllodromiinae (Figure 15A). Inter-ocular space is sharply angled creating a carina that begins where the compound eye meets the antennae. The antennal pits are closer together than the eyes. Eyes are prominent and appear to bulge the head laterally. Facial grooves spanning from the posterior portion of the eye towards the mouthparts are prominent. Coloration on head is brown-orange overall with a slightly lighter, less brown, patch above and below the carina. Ocellar spots are either absent or not readily visible.

The pronotum is colored with a dark black region taking up the major two fifths of the medial area. The black area is opaque and reaches forward to the anterior margin but just stops short of completion in the posterior eighth of the segment. The black region is nearly rectangular, slightly rounded anteriorly and widened posteriorly (Figure 15B). Bordering the black region laterally and posteriorly are translucent regions colored brown-orange similar to the remainder of the body.

Meta- and meso-thoracic segments are both strongly lobed, presumably due to the developing wings within. Color is orange-brown overall with small amounts of black on the tips of the posterior pair of wing pads. Legs are light in color with a slight orange tinge overall. Dark regions are present on the medial side of the base of the fore-coxae.

The ventro-anterior margin of the fore-femur have five (right) or eight (left) large piliform spines basally followed by 27 (right) and 20 (left) shorter piliform spines, which are then each followed by one larger piliform spine and finally one large distal spine that is not piliform. Arolia are large and extend beyond the tips of the pretarsal claws on all legs. Claws are symmetrical and unspecialized.

Both the venter and dorsum of the abdomen is the same orange-brown color as the remainder of the body, but with a slightly redder tinge. Soft black color borders the abdomen laterally and posteriorly.

The dorsal abdomen is mostly glabrous. Hairs that are present are most dense laterally and on segments five and six. Ventral abdomen is glabrous as well, with fewer hairs than on the dorsal side and no regions with any dense pubescence. Supra-anal plate is unspecialized and broadly subtrapezoidal or triangular. Subgenital plate is broadly subtrapezoidal with the posterior margin being broader than that of the subgenital plate. The posterior margin of the subgenital plate is not perfectly uniform and conforms around two large styli. Styli are equal in length to the entire subgenital plate. Their width is equal to half of the length of the visible portion of the styli.

Genetic information and evolutionary placement. Evangelista et al. (2014) recovers this sequence as being most closely related to a species reported as "Ectobiidae sp. 6" with 75\% bootstrap support. This species is identified above as Chorisonuera inversa Hebard, 1926. Hebard hypothesized that these are closely related genera (Hebard 1921a) and we can now say that genetic data supports this hypothesis. We cannot definitively say, however, that they are sister taxa because of incomplete phylogenetic 

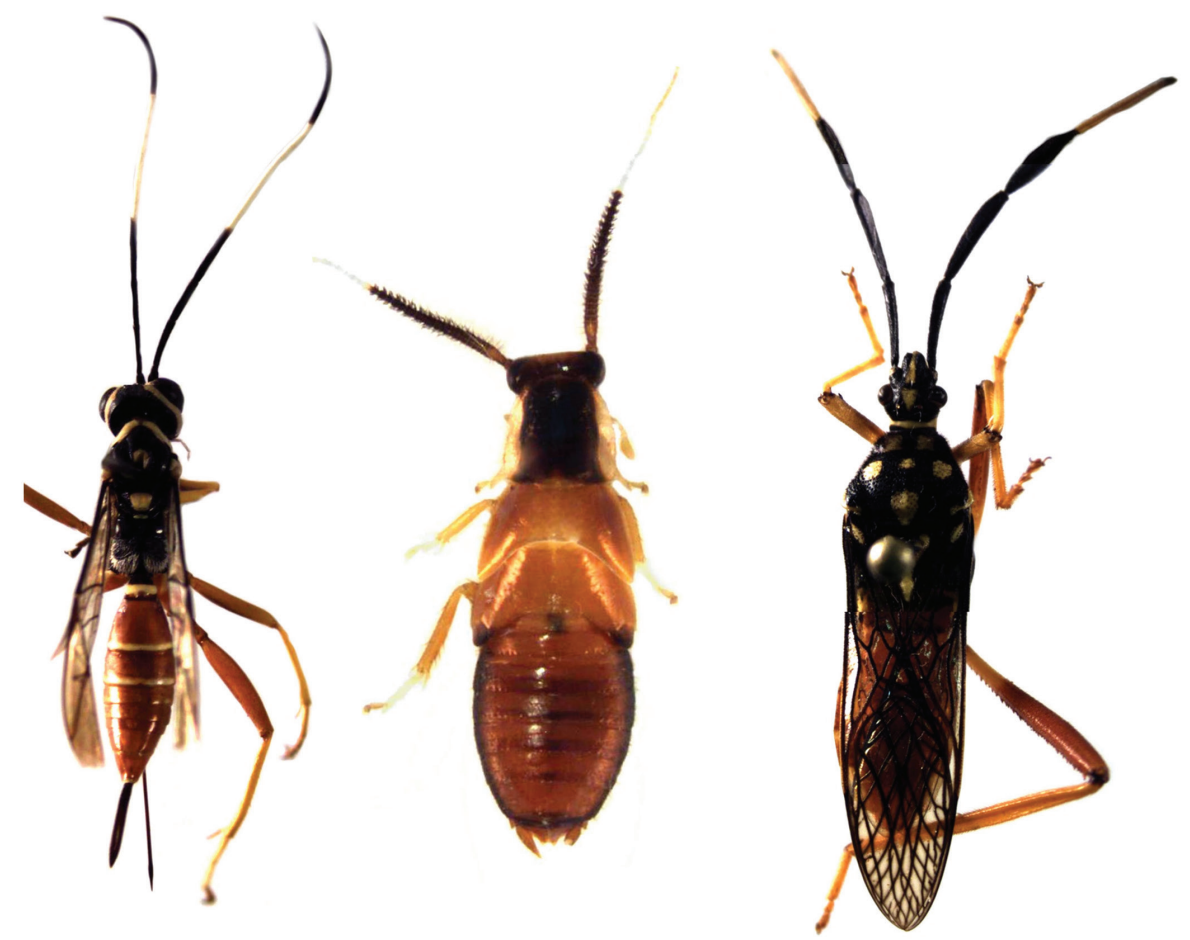

Figure 16. Comparison of overall body coloration of three sympatric species (Left: Ichneumonidae, Middle: Calhypnorna sp. "Aguyana", Right: Reduviidae) from northern Guyana. Calhypnorna sp. shares the orange hind section and dark forward section with the other two insects. Additionally, the antennae of the cockroach composed of: a white band shared with the wasp; an orange band shared with the assassin bug; and a black base share among all. Photos are not to scale.

sampling in this tree. Thus, we follow Hebard (1921a) and not Beccaloni (Beccaloni 2007) and consider this to be in the Psuedophylodromiinae.

Known geographical distribution of Calhypnorna. Guyana (new record), Para Brazil, Bolivia and Panama.

Collection/ecological information. This specimen was found crawling through a benab. The only individual of this species observed in the field was the one collected and described here. Given that our overall collecting effort was significant (>1000 individuals of Blattodea s.s.) and we only found a single individual of Calhypnorna sp. A, we consider this species to be quite rare.

Previous work (Shelford 1912) has cited species of this genus as being beetle mimics. However, we observed no beetle model in the field that this species may have been mimicking. We did notice a similarity in body coloration of a wasp and Hemipteran sympatric with this conspicuously colored Blattodea (Figure 16).

Notes on historical records of this genus. The genus Calhypnorna Saussure \& Zehntner, 1893 was originally established as a subgenus of Hypnorna Stål, 1860. It was then given generic status by Kirby (1904). The genera Calhypnorna, Hypnorna, 
Hypnornoides Rehn, 1917 and Euhypnorna Hebard, 1921 are thought to be closely related (Hebard 1921). These are known from a number of regions (Para and Rio de Janiero Brazil, Bolivia and Panama) but there are no records from the Guiana Shield. Therefore, a new record of this species from the coastal rainforests of Guyana is geographically disjointed from all other records of these taxa. On this basis alone, we might distinguish this specimen as a new species. However, since our lone specimen is a juvenile, we have limited morphological basis for differentiating this from known taxa. We refrain from establishing this as new species until adult specimens can be found but we still give a synopsis of the biological traits of this specimen. This new record extends the potential range of Calhypnorna Saussure \& Zehntner, 1893 and it has now been recorded from Para Brazil (south of Amazon), Bolivia, Panama, and Guyana (new record).

\section{Cockroach fauna of the Guyana Shield: Summary}

The checklist (Table 1) contains 5 families, 18 subfamilies, 79 genera, and 234 species. French Guiana and Suriname contribute the most to this richness, with 151 and 136 species respectively (Figure 17). The surprisingly low number of records from Guianan Venezuela, Roraima and Amapa Brazil (Figure 17) are most definitely due to an historical under sampling in these regions.

When pooling and examining the range data for all the taxa (Figure 18) we see that, as expected, small ranges are most common among species. This is also true when pooling taxa together into genera, although these range sizes are larger overall. 85 species $(36 \%)$ and 20 genera $(25 \%)$ are limited to a single region while 36 species $(15 \%)$ and 24 genera $(30 \%)$ are represented in four or more regions. Small ranges $(<4$ regions) are no longer the majority when lumping species into subfamilies or families.

The highest rates of endemism are seen in Guianan Venezuela, Amapa Brazil and French Guiana (Figure 19). However, we believe these values to be inaccurate due to lack of sampling. Compared on a pairwise basis, Guyana, Suriname and French Guiana had a high proportion of shared fauna (Figure 20). These are each proximal to each other and centrally located, thus their faunal similarity is expected. Roraima showed a high number of its own species shared among each other region. However, most of the species recorded from Roraima are circumtropical taxa and the region is severely under sampled.

Most of the species in the checklist have neotropical distributions. There were few taxa listed with distributions that may be considered circumtropical or cosmopolitan: Blatta orientalis Linnaeus, Neostylopygia rhombifolia (Stoll), Periplaneta americana (Linnaeus), P. australasiae (Fabricius), P. brunnea Burmeister, Holocompsa nitidula (Fabricius), Phoetalia pallida (Brunner von Wattenwyl), P. circumvagans (Burmeister), Nauphoeta cinerea (Olivier), Rhyparobia maderae (Fabricius), Panchlora nivea (Linnaeus), Pycnoscelus surinamensis (Linnaeus), Blattella germanica (Linnaeus), Supella longipalpa (Fabricius). Most of these may be considered non-native, or adventive. 


\section{Discussion}

The majority of records used to compile the checklist were lacking in specific biological, geographic or ecological information. Most historical records we encountered only gave general collection locales within their respective country. GPS information was non-existent for nearly all records.

We present eight new species records for Guyana. This includes one genus new to the entire shield (Calhypnorna Saussure \& Zehntner, 1893) and one new species (Xestoblatta berenbaumae). Given the somewhat high local richness of cockroaches (Evangelista et al. 2014) in one small plot compared to the richness of the entire country (Figure 17) we believe that much of this country's diversity has yet to be discovered.

Among the regions considered here, Guyana and Amapa are moderately well sampled. Guianan Venezuela, and Roraima Brazil are sampled especially poorly and our knowledge of the Blattodea of these regions is very much preliminary. In contrast, French Guiana and Suriname are some of the most well sampled cockroach faunas in all the neotropics, ranking as the $2^{\text {nd }}$ and $6^{\text {th }}$ most species dense regions respectively (Table 4). The most well sampled region in the neotropics, Rio de Janeiro, has a species density of 0.01 species per square mile (Table 4). If we consider this value as being typical of true species density, which is purely speculation, then no other neotropical region has been sampled thoroughly.

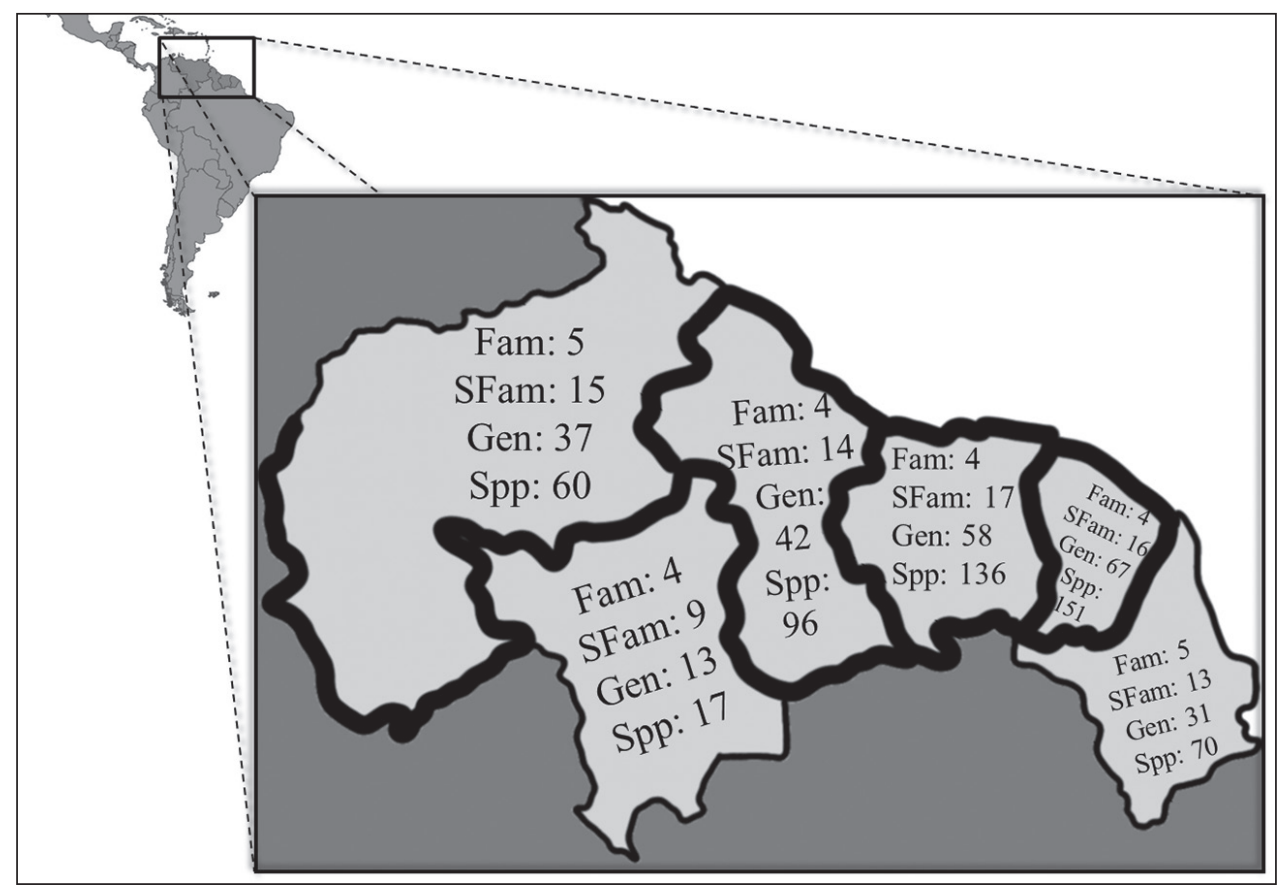

Figure I7. Known richness of cockroach fauna at different taxonomic levels for six regions of the Guiana Shield. 


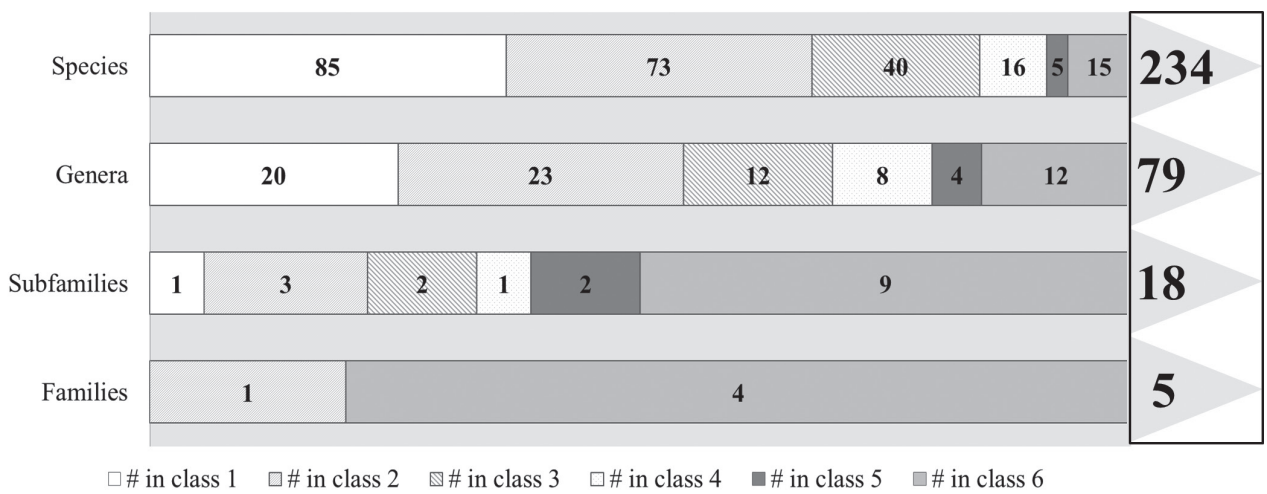

Figure 18. Extent of range for cockroach taxa. Classes represent the number of regions a taxon was present in: present in only one region - class one; present in all six regions - class 6; etc. Total number of taxa for each level shown on the right.

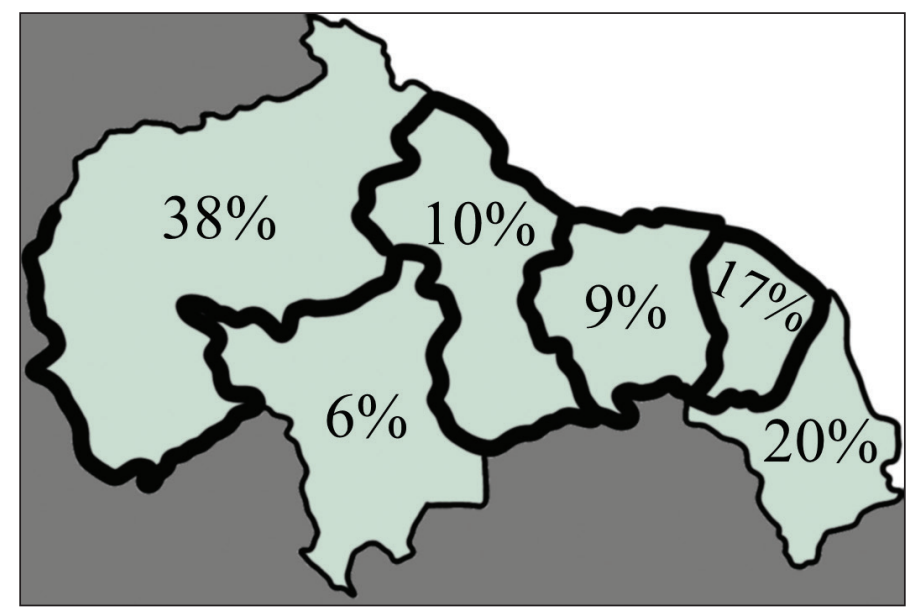

Figure 19. Proportion of cockroach fauna endemic to a region. Endemism is only referred to within the context of the shield.

The levels of endemism we see (Figure 19) are surprisingly low compared with other known rates of endemism for the Guiana Shield (Funk et al. 2007; Hollowell and Reynolds 2005; Kelloff and Funk 2004; Naka 2011). One possible explanation would simply be that cockroaches have low rates of tropical endemism. However, this is contradicted by other cockroach faunas showing much higher rates of endemism (e.g. $-60 \%$ of all taxa in Hispaniola; Gutierrez and Perez-Gelabert 2000). The alternate explanation is that there is a collection bias for taxa with broad ranges. This could be true if geographic sampling is very sparse, which may be the case. The levels of endemism we report (Figure 19) are actually higher than what they are in reality, since we only considered strictly Guianan regions. There are likely a few species that appear endemic when only considering these regions but by expanding the geographic scope 


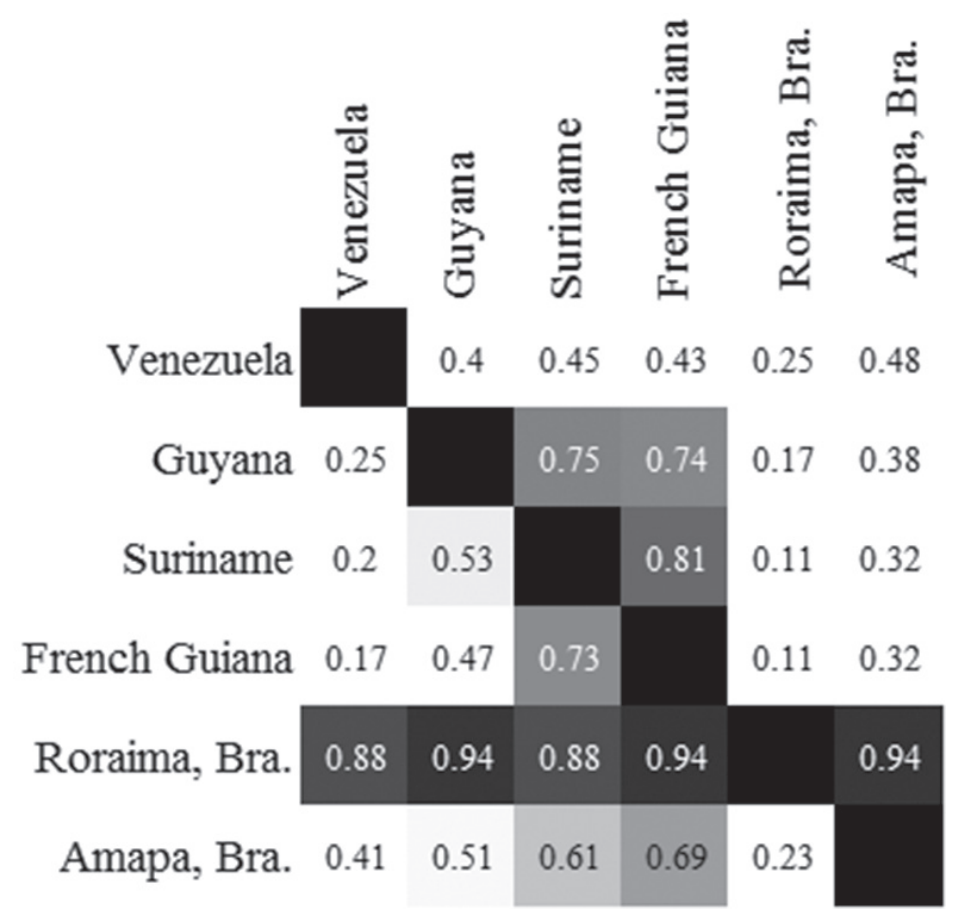

Figure 20. Proportion of fauna in a region (left) shared with each other region (top). Values greater than .5 are shaded by magnitude. The three central regions (Guyana, Suriname and French Guiana) have a high degree of similarity with each other.

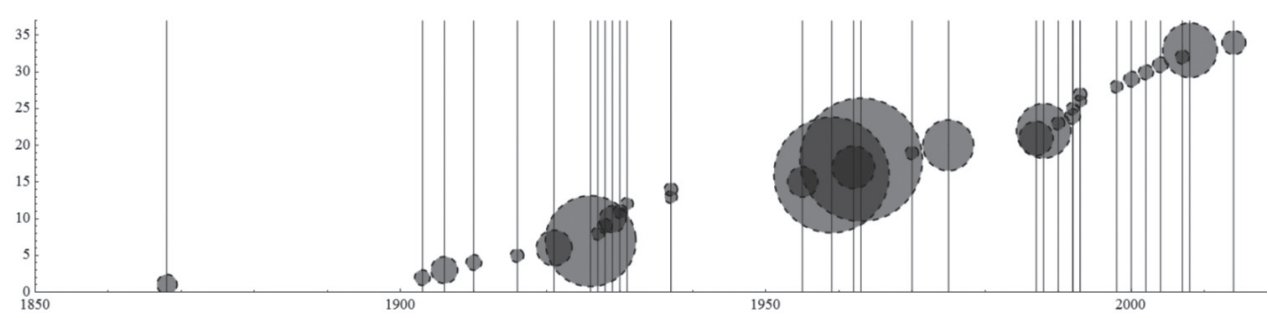

Figure 2I. Studies contributing to the checklist of cockroaches of the Guiana Shield. The year of publication of each source plotted against the order in which they were published. The present study, the 34th, is the final circle in the top right. The radius of the circles represents the relative number of times that study is cited in the checklist.

we would find that they are actually not Guianan endemics (e.g. also being present in Trinidad, Colombia or other parts of Brazil).

If we didn't already know that under-sampling for cockroaches (Pellens and Grandcolas 2008; Roth 2003) and other insects (Erwin 1982; Stork 1993) was generally problematic, we could infer this based on a number of clues in our data. First, as mentioned previously, an estimate of total species richness of cockroaches for one small plot in northern Guyana nearly matches the recorded richness of the entire country 
Table 4. The ten regions of the Neotropics with the highest known cockroach richness per unit area.

\begin{tabular}{c|c|c|c|c}
\hline Region & Size $\left(\mathbf{m i}^{\mathbf{2}}\right)$ & \# of spp. & $\mathbf{s p p} / \mathbf{m i}^{\mathbf{2}}$ & Source \\
\hline Rio de Janeiro, Brazil & 16,871 & 169 & 0.0100 & (Pellens and Grandcolas 2008) \\
\hline French Guiana & 32,253 & 151 & 0.0047 & - \\
\hline Panama & 29,118 & 118 & 0.0041 & (Beccaloni 2007) \\
\hline Costa Rica & 19,730 & 72 & 0.0036 & (Beccaloni 2007) \\
\hline Hispaniola & 29,530 & 86 & 0.0029 & (Perez-Gelabert 2008) \\
\hline Continental Ecuador & 46,444 & 114 & 0.0025 & (Vidlicka 2013) \\
\hline Suriname & 63,039 & 136 & 0.0022 & - \\
\hline Cuba & 42,426 & 85 & 0.0020 & - \\
\hline Amapa, Brazil & 55,141 & 70 & 0.0013 & - \\
\hline Guyana & 83,000 & 96 & 0.0012 &
\end{tabular}

(Evangelista et al. 2014). Furthermore, there are 20 cases of species with unusual distributions (Table 5), where it is absent from a region but recorded from neighboring regions. Without evidence to the contrary, the simplest explanation for these distribution "holes" is inadequate sampling. Finally, although specific locality information is severely lacking for most records, those that are recorded do not represent effective spatial sampling, and most records are from coastal areas of major rivers. Finally, the number of species per region is significantly lower than that of better sampled but less diverse taxa such as Odonata (Checklist of Odonata of the Guiana Shield 2012; Garrison et al. 2006, 2010).

Although there is clearly a great under-sampling of cockroaches from this region, we cite 34 publications that contributed to this checklist, including the present (Figure 21). The earliest source was from 1868 (Walker 1868). Most of the publications contributing to the checklist were published between 1900 and 1940. Morgan Hebard, Isolda Rocha e Silva Albuquerque, Ashley Gurney and James Rehn contributed the most through primary taxonomic publications and species descriptions (in particular see Hebard 1926; Rehn 1930; Rocha E Silva Albuquerque and Gurney 1962). Karlis Princis, J. Bonfils and Conrad F.A. Bruijning were also important in these capacities but more-so through their own published checklists. Jaime Perez and J. Bonfils were also great contributors to the fauna of Venezuela and French Guiana. Similarly, Roseli Pellens was an important contributor to the knowledge of the two Brazilian regions through her checklist. Philippe Grandcolas was also an instrumental author through this same checklist, as well as other primary taxonomic publications. The three most cited papers in the checklist are Princis' "Orthopterum Catalogus" (148 citations), Bruijning's "The Blattidae of Surinam" (138 citations), and Hebard's "The Blattidae of French Guiana" (105 citations) (Figure 21). It is worth restating that, although they are invaluable authors, Princis' and Bruijning's contributions were mainly through synthesizing work done by others. The significance of Hebard's contribution to the knowledge of the Guianan fauna through "The Blattidae of French Guiana", in which he alone described 53 new species, cannot be understated. 
Table 5. Recorded (o and + ) and projected (p) presences of cockroaches from the Guiana Shield. VEN - Combined data from Amazonas, Bolivar and Delta Amacuro Venezuela; GUY - Guyana; SUR - Suriname; FG - French Guiana; Rora BRA - Roraima, Brazil. Amapa BRA - Amapa, Brazil. Projected occurrences are expectations of species presence based on confirmed presence in neighboring regions. Data used to determine this is taken from the checklist (Table 1) and other sources (see Table 1 for citations for these species).

\begin{tabular}{|c|c|c|c|c|c|c|}
\hline & $V E N$ & $G U Y$ & SUR & $F G$ & Rora BRA & Amapa BRA \\
\hline \multicolumn{7}{|l|}{ Blaberidae } \\
\hline \multicolumn{7}{|l|}{ Blaberinae } \\
\hline Blaberus colosseus & $\mathbf{p}$ & o & $\mathbf{p}$ & o & & \\
\hline Blaberus craniifer & $\mathbf{p}$ & o & $\mathbf{p}$ & o & & \\
\hline \multicolumn{7}{|l|}{ Epilamprinae } \\
\hline Epilampra azteca & o & $\mathbf{p}$ & o & o & & \\
\hline Epilampra maculicollis & & o & $\mathbf{p}$ & o & & \\
\hline \multicolumn{7}{|l|}{ Panchlorinae } \\
\hline Panchlora bidentula & o & $\mathbf{p}$ & o & o & & \\
\hline \multicolumn{7}{|l|}{ Zetoborinae } \\
\hline Thanatophyllum akinetum & & + & $\mathbf{p}$ & o & & \\
\hline \multicolumn{7}{|l|}{ Ectobiidae } \\
\hline \multicolumn{7}{|l|}{ Anaplectinae } \\
\hline Anaplecta subsignata & o & $\mathbf{p}$ & o & o & & o \\
\hline Maraca fossata & o & $\mathbf{p}$ & o & o & & \\
\hline \multicolumn{7}{|l|}{ Blattellinae } \\
\hline Cahita misella & $\mathbf{p}$ & $\mathbf{p}$ & $\mathbf{p}$ & o & & \\
\hline Chromatonotus notatus & $\mathbf{p}$ & $\mathbf{p}$ & o & o & & \\
\hline Eudromiella ineopectata & & o & $\mathbf{p}$ & o & & \\
\hline Xestoblatta nyctiboroides & & o & $\mathbf{p}$ & o & & \\
\hline Xestoblatta agautierae & & + & $\mathbf{p}$ & o & & \\
\hline \multicolumn{7}{|l|}{ Pseudophyllodromiinae } \\
\hline Anisopygia decora & & o & $\mathbf{p}$ & o & & \\
\hline Arawakina frontalis & & o & $\mathbf{p}$ & o & & \\
\hline Chorisoneura gatunae & $\mathbf{p}$ & $\mathbf{p}$ & o & o & & \\
\hline Euphyllodromia chopardi & & o & $\mathbf{p}$ & o & o & o \\
\hline Neoblattella guianae & & o & $\mathbf{p}$ & o & & o \\
\hline Sciablatta poecila & & o & $\mathbf{p}$ & o & & \\
\hline Trioblattella callosoma & & $\mathrm{o}$ & $\mathbf{p}$ & o & & o \\
\hline
\end{tabular}

\section{Conclusions}

This checklist of Blattodea s.s. of the Guiana Shield, showing 234 species, is the most comprehensive to date. It is also functions as the first true checklist of cockroaches of Guyana, as all previous sources severely fall short of listing even the modest number of species we record here. Given the large number of species found in the small country of French Guiana, we see that the Guiana Shield may be one of world's hotspots of 
biodiversity for cockroaches. However, sampling is still severely lacking. What little sampling has been done in the Guianas was mostly completed before 1960. There are huge gaps to fill in, and until they are we will be unable to adequately address most questions about the nature and origins of cockroach biodiversity.

\section{Acknowledgements}

Thank you to Dr. Sonia Maria Lopes, Dr. Esteban Gutierrez for assistance and mentoring in identification of some of the species reported here as well as for reviewing this manuscript. Thanks and appreciation is given to Dr. Peter Vrsansky for critically reviewing the manuscript as well. We would also like to acknowledge all those individuals listed as collectors of the specimens with special thanks to Joseph Evangelista, and Ian Biazzo. Ms. Kimberly Guzman contributed photos and collection management. Finally, Dominic Evangelista would like to thank Dr. May Berenbaum for her generous monetary donation to his thesis work. This work was also funded by Rutgers RUFAIR award, Ware's Rutgers start-up funds, and the Smithsonian Biodiversity of the Guianas project (BDG 204).

\section{References}

Alexander EE, Bassett Y, Charles E, De Dijn BPE, Forget P-M, Hammond DS, Hounter NC, Pons TL, Rijkers T, Rose SA, Springate ND (2005) Tropical Forests of the Guiana Shield: ancient forests in a modern world. CABI Publishing, Cambridge, 535 pp.

Baaren Jv, Deleporte P, Grandcolas P (2002) Cockroaches in French Guiana Icteridae birds nests. Amazoniana 17: 243-248.

Beccaloni G (2014) Cockroach Species File Online. Version 5.0. http://cockroach.speciesfile. org/ [Accessed June 2014]

Beccaloni G, Eggleton P (2011) Taxonomy of Blattodea. Zootaxa 3148: 199-200.

Beccaloni G, Eggleton P (2013) Order: Blattodea. In: Zhang Z-Q (Ed.) Animal Biodiversity: An Outline of Higher-level Classification and Survey of Taxonomic Richness. Zootaxa 3703: 46-48. doi: 10.11646/zootaxa.3703.1.10

Bonfils J (1975) Blattoptera [Orthopteroidea] récoltés en Guyane Française par la mission du muséum national d'histoire naturelle. Annales de la Société entomologique de France Medecine 11: 29-62.

Bonfils J (1987) Les Blattes (Dictyoptera: Blattaria) du Venezuela. Fauna hipogea y hemiedáfica de Venezuela y de otros paises de América del Sur. Editura Academiei Republicii Socialists Romania, Bucurest, 157-164.

Bruijning CFA (1959) The Blattidae of Surinam. Studies on the Fauna of Suriname and other Guyanas 2: 1-103.

Checklist of Odonata of the Guiana Shield (2012) Checklist of Odonata of the Guiana Shield. http://www.libellen.org/suriname/7checklist/Checklist_Guiana_Shield4.htm 
Djernaes M, Klass K-D, Picker MD, Damgaard J (2012) Phylogeny of cockroaches (Insecta, Dictyoptera, Blattodea), with placement of aberrant taxa and exploration of out-group sampling. Systematic Entomology 37: 65-83. doi: 10.1111/j.1365-3113.2011.00598.x

Djernaes M, Klass K-D, Eggleton P (2014) Identifying possible sister groups of Cryptocercidae+Isoptera: A combined molecular and morphological phylogeny of Dictyoptera. Molecular Phylogenetics and Evolution. doi: 10.1016/j.ympev.2014.08.019 [in press]

Erwin TL (1982) Tropical forests: their richness in Coleoptera and other Arthropod species. The Coleopterists Bulletin 36: 74-75.

Evangelista DA, Bourne G, Ware JL (2014) Species richness estimates of Blattodea s.s. (Insecta: Dictyoptera) from northern Guyana vary depending upon methods of species delimitation. Systematic Entomology 39: 150-158. doi: 10.1111/syen.12043

Evangelista DA, Buss L, Ware JL (2013) Using DNA barcodes to bonfirm the presence of a new invasive cockroach Pest in New York City. Journal of Economic Entomology 106: 2275-2279. doi: 10.1603/ec13402

Funk VA, Berry P, Kelloff C, Alexander SN (2007) Checklist of the plants of the Guiana Shield (Venezuela: Amazonas, Bolivar, Delta Amacuro; Guyana, Surinam, French Guiana). Contributions from the United States National Herbarium 55: 1-584.

Garrison R, von Ellenrieder N, Louton JA (2006) Dragonfly Genera of the New World. Johns Hopkins University Press, Blatimore, 368 pp.

Garrison R, von Ellenrieder N, Louton JA (2010) Damselfly Genera of the New World. Johns Hopkins University Press, Blatimore, 490 pp.

Grandcolas P (1990) Descriptions de nouvelles Zetoborinae guyanaises avec quelques remarques sur la sous-famille. Bulletin de la Société Entomologique de France 95: 241-246.

Grandcolas P (1992a) Evolution du mode de vie, repartition et nouveaux taxons dans le genre Xestoblatta Hebard, 1916 (Dictyoptera, Blattellidae, Blattellinae). Revue Francaise D’Entomologie 14: 155-168.

Grandcolas P (1992b) Paradicta n. gen. et Neorhicnoda n. gen., deux nouvaeux genres de Blaberinae (Dict., Blattaria, Blaberidae). Bulletin de la Société Entomologique de France 97: 7-15.

Grandcolas P (1993a) Le genre Paramuzoa Roth, 1973: sa repartition et un cas de xylophagie chez les Nyctiborinae (Dictyoptera, Blattaria). Bulletin de la Société Entomologique de France 98: 131-138.

Grandcolas P (1993b) Monophylie et structure phylogenetique des [Blaberinae+Zetoborinae+ Gyninae+Diplopterinae] (Dictyoptera:Blaberidae). Bulletin de la Société Entomologique de France 29: 195-222.

Gurney AB (1939) A revision of the neotropical genus Xestoblatta Hebard (Orthoptera; Blattidae; Pseudomopinae). Proceedings of the Entomological Society of Washington 41: 97-128.

Gutierrez E (1995) Annotated checklist of cuban cockroaches. Transactions of the American Entomological Society 121: 65-85.

Gutierrez E, Perez-Gelabert D (2000) Annotated checklist of Hispaniolan Cockroaches. Transactions of the American Entomological Society 126: 433-446.

Hebard M (1916) Studies in the group Ischnopterites (Orthoptera, Blattidae, Pseudomopinae). Transactions of the American Entomological Society 42: 337-383. 
Hebard M (1921a) A note on Panamanian Blattidae with the description of a new genus and two new species. Entomological News 32: 161-169.

Hebard M (1921b) South American Blattidae from the Museum National d'Histoire Naturelle, Paris, France. Proceedings of the Academy of Natural Sciences of Philadelphia 73: 193-304.

Hebard M (1926) The Blattidae of French Guiana. Proceedings of the Academy of Natural Sciences of Philadelphia 78: 135-244.

Hebard M (1929) Previously unreported tropical American Blattidae (Orthoptera) in the British Museum. Transactions of the American Entomological Society 55: 345-388.

Hebard M (1931) Die Ausbeute der deutschen Chaco-Expedition 1925/26 - Orthoptera. Zeitschrift fur systematiische Insektenkunde 10: 257-285.

Hollowell T, Reynolds RP (2005) Checklist of the terrestrial vertebrates of the Guiana Shield. Bulletin of the Biological Society of Washington 13: 1-93. doi: 10.2988/0097-0298(200 5) $13[1: \mathrm{I}] 2.0 . \mathrm{CO} ; 2$

INFINITY Camera Software (2013) INFINITY Camera Software. Lumenera Corporation.

Inward D, Beccaloni G, Eggleton P (2007) Death of an order: a comprehensive molecular phylogenetic study confirms that termites are eusocial cockroaches. Biology Letters 3: 331-335. doi: $10.1098 /$ rsbl.2007.0102

Kelloff CL, Funk VA (2004) Phytogeography of the Kaieteur Falls, Potaro Plateau, Guyana: Floral distributions and affinities. Journal of Biogeography 31: 501-513. doi: 10.1046/j.03 05-0270.2003.01038.x

Kirby WF (1904) A synonymic catalogue of Orthoptera. Order of the Trustees of the British Museum, London. doi: 10.5962/bhl.title.6745

Lopes SM, de Oliveira EH (2004) Two New Species of Helgaia (Blattaria: Blattellidae) from Brazil with Description of Male and Female Genitalia of Helgaia serrana and Keys to the Species. Studies on Neotropical Fauna and Environment 39: 57-61. doi: 10.1080/01650520412331270981

Lopez-Osorio F, Miranda-Esquivel DR (2010) A phylogenetic approach to conserving Amazonian biodiversity. Conservation Biology 24: 1359-1366. doi: 10.1111/j.15231739.2010.01482.x

Naka LN (2011) Avian distribution patterns in the Guiana Shield: implications for the delimitation of Amazonian areas of endemism. Journal of Biogeography 38: 681-696. doi: 10.1111/j.1365-2699.2010.02443.x

Nickle D (1984) Epilampra maya Rehn, a Central American cockroach newly established in the United States (Blattodea; Blaberidae; Epilamprinae). The Florida Entomologist 67: 487-489. doi: 10.2307/3494732

Pellens R, Grandcolas P (2008) Catalogue of Blattaria (Insecta) from Brazil. Zootaxa 1709: $1-109$.

Perez-Gelabert D (2008) Arthropods of Hispaniola (Dominican Republic and Haiti): A checklist and bibliography. Zootaxa 1831: 1-530.

Perez JR (1988) Revision taxonomica de las cucarachas (Blattaria, Dictioptera) de Venezuela. Boletin de la Direccion de Malariologia Y Saneamiento Ambiental 28: 128-149. 
Peterson W, Cobb K (2009) First Record of the Turkestan Cockroach, Blatta lateralis (Walker), in Georgia (USA). Journal of Entomological Sciences 44: 415-416.

Princis K (1948) Uber einige neue bzw. wenig bekannte Blattarien aus dem Naturhistorischen Reichmuseum zu Stockholm. Arkiv for Zoologi 41: 1-23.

Princis K, Kevan DKM (1955) Cockroaches (Blattariae) from Trinidad, B.W.I., with a few records from other parts of the Caribbean. Opuscula Entomologica 20: 149-169.

Princis K (1963) Orthopterum Catalogus. W. Junk, 's-Gravenhage, 246 pp.

Rehn JA (1903) Studies in American Blattidae. Transactions of the American Entomological Society 29: 259-290.

Rehn JA (1906) Records and descriptions of non-saltatorial Orthoptera from British Guiana. Proceedings of the Academy of Natural Sciences of Philadelphia 58: 262-278.

Rehn JA (1917) On Orthoptera from the Vicinity of Rio de Janeiro, Brazil. Transactions of the American Entomological Society 43: 335-363.

Rehn JA, Hebard M (1927) The Orthoptera of the West Indies Number 1. Blattidae. Bulletin of the American Museum of Natural History 54: 1 - 320.

Rehn JA (1928) New or little known neotropical Blattidae (Orthoptera): Number one. Transactions of the American Entomological Society 54: 125-194.

Rehn JA (1930) New or little known neotropical Blattidae (Orthoptera): Number two. Transactions of the American Entomological Society 56: 19-71.

Rehn JA (1937a) New or little known neotropical Blattidae (Orthoptera): Number four. Transactions of the American Entomological Society 63: 207-258.

Rehn JA (1937b) A new species of Blattidae from British Guiana. The annals and magazine of natural history 20: 197-203. doi: 10.1080/00222933708655333

Rocha E Silva Albuquerque I, Gurney AB (1962) Insecta Amapaensia - Orthoptera: Blattoidea. Studia Entomologia 5: 235-255.

Roth LM (1969) The male genitalia of Blattaria. I. Blaberus spp. (Blaberidae: Blaberinae). Psyche 76: 217-250. doi: 10.1155/1969/31394

Roth LM (1970a) The male genitalia of Blattaria. IV. Blaberidae: Blaberinae. Psyche 77: 308-342.

Roth LM (1970b) The male genitalia of Blattaria. V. Epilampra spp. (Blaberidae: Epilamprinae). Psyche 77: 436-486. doi: 10.1155/1970/46805

Roth LM (2003) Systematics And Phylogeny Of Cockroaches (Dictyoptera: Blattaria). Oriental Insects 37: 1-186. doi: 10.1080/00305316.2003.10417344

Roth LM, Gutierrez E (1998) The cockroach genus Colapteroblatta, its Synonyms Poroblatta, Acroporoblatta, and Nauclidas, and a new species of Litopeltis (Blattaria: Blaberidae, Epilamprinae). Transactions of the American Entomological Society 124: 167-202.

Saussure Hd, Zehntner L (1893) Insecta. Orthoptera. Biologia Centrali-Americana 1: 1-285.

Shelford R (1910) Orthoptera: Family Blattidae: Subfamily Epilamprinae. Genera insectorum 101: 1-21.

Shelford R (1912) Mimicry amongst the Blattidae; with a revision of the genus Prosoplecta Sauss. and the description of a new genus. Proceedings of the Zoological Society of London 82: 358-378. doi: 10.1111/j.1469-7998.1912.tb07022.x

Stork NE (1993) How many species are there? Biodiversity and Conservation 2: 215-232. doi: 10.1007/BF00056669 
Velez A (2008) Checklist of Colombian cockroaches (Dictyoptera, Blattaria). Biota Colombiana 9: 21-38.

Walker F (1868) Catalogue of Specimens of Blattariae in the Collection of the British Museum. London, 237 pp.

Ware JL, Litman J, Klass K-D, Spearman LA (2008) Relationships among the major lineages of Dictyoptera: the effect of outgroup selection on dictyopteran tree topology. Systematic Entomology 33: 429-450. doi: 10.1111/j.1365-3113.2008.00424.x

Wolfram Research (2012) Mathematica: version 9.1. Wolfram Research Inc., Champaign, Illinois. https://www.wolfram.com/mathematica/ 